\title{
Fully Discrete Analysis of a Discontinuous Finite Element Method for the Keller-Segel Chemotaxis Model
}

\author{
Yekaterina Epshteyn*and Ahmet Izmirlioglu ${ }^{\dagger}$
}

\begin{abstract}
This paper formulates and analyzes fully discrete schemes for the two-dimensional Keller-Segel chemotaxis model. The spatial discretization of the model is based on the discontinuous Galerkin methods and the temporal discretization is based either on Forward Euler or the second order explicit total variation diminishing (TVD) Runge-Kutta methods. We consider Cartesian grids and prove optimal fully discrete error estimates for the proposed methods. Our proof is valid for pre-blow-up times since we assume boundedness of the exact solution.
\end{abstract}

AMS subject classification: 65M60, 65M12, 65M15, 92C17, 35K57

Key words: Keller-Segel chemotaxis model, convection-diffusion-reaction systems, discontinuous Galerkin methods, Forward Euler, Runge-Kutta, NIPG, IIPG, and SIPG methods, Cartesian meshes.

\section{Introduction}

The goal of this work is to formulate and analyze fully discrete discontinuous Galerkin (DG) methods for the solution of the two-dimensional (2-D) Keller-Segel chemotaxis model, [11, 26, $27,28,33,34]$. The underlying spatial discretization of the model is based on the methods proposed recently in [18] and the temporal discretization is based either on Forward Euler or the second order explicit total variation diminishing (TVD) Runge-Kutta methods.

In this paper, we consider the classical formulation of the Keller-Segel system [11], which can be written in the dimensionless form as

$$
\left\{\begin{array}{l}
\rho_{t}+\nabla \cdot(\chi \rho \nabla c)=\Delta \rho, \quad(x, y) \in \Omega, t>0 \\
c_{t}=\Delta c-c+\rho
\end{array}\right.
$$

subject to the Neumann boundary conditions:

$$
\nabla \rho \cdot \mathbf{n}=\nabla c \cdot \mathbf{n}=0, \quad(x, y) \in \partial \Omega .
$$

Here, $\rho(x, y, t)$ is the cell density, $c(x, y, t)$ is the chemoattractant concentration, $\chi$ is a chemotactic sensitivity constant, $\Omega$ is a bounded domain in $\mathbb{R}^{2}, \partial \Omega$ is its boundary, and $\mathbf{n}$ is a unit normal

*Department of Mathematical Sciences, Carnegie Mellon University, Pittsburgh, PA, 15213, rina10@andrew. cmu . edu

$\dagger$ Department of Mathematics, University of Pittsburgh, Pittsburgh, PA 15260; ahist1@pitt.edu 
vector. The system (1.1) is the basic step in the modeling of many biological processes. The Keller-Segel model (1.1) can be generalized to better describe the reality by taking into account some other factors such as growth and death of cells, presence of the food and other chemicals in the system, etc.

It is well-known that solutions of the classical Keller-Segel system may blow up in finite time, see, e.g., $[24,25]$ and references therein. This blow-up represents a mathematical description of a cell concentration phenomenon that occurs in real biological systems, see, e.g., [1, 6, 8, 9, 15, 35].

Capturing blowing up solutions numerically is a challenging problem. A finite-volume, [21], and a finite-element, [32], methods have been proposed for a simpler version of the Keller-Segel model,

$$
\left\{\begin{array}{l}
\rho_{t}+\nabla \cdot(\chi \rho \nabla c)=\Delta \rho \\
\Delta c-c+\rho=0
\end{array}\right.
$$

in which the equation for concentration $c$ has been replaced by an elliptic equation using an assumption that the chemoattractant concentration $c$ changes over much smaller time scales than the density $\rho$. A fractional step numerical method for a fully time-dependent chemotaxis system from [39] has been proposed in [40]. However, the operator splitting approach may not be applicable when a convective part of the chemotaxis system is not hyperbolic, which is a generic situation for the original Keller-Segel model as it was shown in [10], where the finitevolume Godunov-type central-upwind scheme was derived for (1.1) and extended to some other chemotaxis and haptotaxis models.

The high-order discontinuous Galerkin method that is investigated here is based on the method proposed in [18]. The DG methods have recently become increasingly popular thanks to their flexibility for adaptive simulations, suitability for parallel computations, applicability to problems with discontinuous coefficients and/or solutions, and compatibility with other numerical methods. These methods have been successfully applied to a wide variety of problems, ranging from the solid mechanics to the fluid mechanics (see, e.g., [13, 14, 12, 19, 20, 22, 38] and references therein). Furthermore, the DG methods are among the methods that can be used for real biomedical problems, which are often considered in complex domains, have discontinuity in the coefficients, and incorporate PDEs of different mathematical nature.

In order to develop high-order DG methods for (1.1) in [18], the Keller-Segel system is rewritten as a system of the nonlinear convection-diffusion-reaction equations by introducing new variables $(u, v):=\nabla c:$

$$
k \mathbf{Q}_{t}+\mathbf{F}(\mathbf{Q})_{x}+\mathbf{G}(\mathbf{Q})_{y}=k \Delta \mathbf{Q}+\mathbf{R}(\mathbf{Q}),
$$

where $\mathbf{Q}:=(\rho, c, u, v)^{T}$, the fluxes are $\mathbf{F}(\mathbf{Q}):=(\chi \rho u, 0,-c, 0)^{T}$ and $\mathbf{G}(\mathbf{Q}):=(\chi \rho v, 0,0,-c)^{T}$, the reaction term is $\mathbf{R}(\mathbf{Q}):=(0, \rho-c,-u,-v)$, the constant $k=1$ in the first two equations in (1.2), and $k=0$ in the third and the fourth equations there.

The methods proposed in [18] are based on three primal DG methods: the Nonsymmetric Interior Penalty Galerkin (NIPG), the Symmetric Interior Penalty Galerkin (SIPG), and the Incomplete Interior Penalty Galerkin (IIPG) methods, $[3,16,36]$. The numerical fluxes in the proposed DG methods are the fluxes developed for the semidiscrete finite-volume central-upwind schemes in [30] (see also [29, 31]). These schemes belong to the family of non-oscillatory central schemes, which are highly accurate and efficient methods applicable to general multidimensional systems of conservation laws and related problems. Like other central fluxes, the central-upwind ones are obtained without using (approximate) Riemann problem solver, which is unavailable for the system under consideration. At the same time, a certain upwinding information - one-sided 
speeds of propagation - is incorporated into the central-upwind fluxes. In [18], Cartesian grids are considered and the continuous in time error estimates are proved for the proposed high-order DG methods under the assumption of boundedness of the exact solution. Some numerical tests that validate the methods are considered in [18] and [17].

In the following section, we introduce our notations, assumptions, and state some standard results. In $\S 3-\S 4$ we recall some results for the continuous in time scheme. In $\S 5-\S 7$ we formulate the explicit schemes and derive the error estimates under assumption of boundedness of the exact solution (some proof details are postponed to Appendix 9). The proof of the error estimates is based on the induction argument which simplifies the analysis significantly since we consider the coupled system of the nonlinear equations.

\section{Assumptions, Notations, and Standard Results}

We denote by $\mathcal{E}_{h}$ a nondegenerate quasi-uniform rectangular subdivision of the domain $\Omega$ (the quasi-uniformity requirement will only be used for establishing the rate of convergence with respect to the polynomials degree). The maximum diameter over all mesh elements is denoted by $h$ and the set of the interior edges is denoted by $\Gamma_{h}$. To each edge $e$ in $\Gamma_{h}$, we associate a unit normal vector $\mathbf{n}_{e}=\left(n_{x}, n_{y}\right)$. We assume that $\mathbf{n}_{e}$ is directed from the element $E^{1}$ to $E^{2}$, where $E^{1}$ denotes a certain element and $E^{2}$ denotes an element that has a common edge with the element $E^{1}$ and a larger index (this simplified element notation will be used throughout the paper). For a boundary edge, $\mathbf{n}_{e}$ is chosen so that it coincides with the outward normal.

The discrete space of discontinuous piecewise polynomials of degree $r$ is denoted by

$$
\mathcal{W}_{r, h}\left(\mathcal{E}_{h}\right)=\left\{w \in L^{2}(\Omega): \forall E \in \mathcal{E}_{h},\left.w\right|_{E} \in \mathrm{P}_{r}(E)\right\},
$$

where $\mathrm{P}_{r}(E)$ is a space of polynomials of degree $r$ over the element $E$. For any function $w \in \mathcal{W}_{r, h}$, we denote the jump and average operators over a given edge $e$ by $[w]$ and $\{w\}$, respectively:

for an interior edge $e=\partial E^{1} \cap \partial E^{2}, \quad[w]:=w_{e}^{E^{1}}-w_{e}^{E^{2}}, \quad\{w\}:=0.5 w_{e}^{E^{1}}+0.5 w_{e}^{E^{2}}$, for a boundary edge $e=\partial E^{1} \cap \partial \Omega, \quad[w]:=w_{e}^{E^{1}}, \quad\{w\}:=w_{e}^{E^{1}}$,

where $w_{e}^{E^{1}}$ and $w_{e}^{E^{2}}$ are the corresponding polynomial approximations from the elements $E^{1}$ and $E^{2}$. We also recall that the following identity between the jump and the average operators is satisfied:

$$
\left[w_{1} w_{2}\right]=\left\{w_{1}\right\}\left[w_{2}\right]+\left\{w_{2}\right\}\left[w_{1}\right] .
$$

For the finite-element subdivision $\mathcal{E}_{h}$, we define the broken Sobolev space

$$
H^{s}\left(\mathcal{E}_{h}\right)=\left\{w \in L^{2}(\Omega):\left.w\right|_{E^{j}} \in H^{s}\left(E^{j}\right), j=1, \ldots, N_{h}\right\}
$$

with the norms

$$
\|w\|_{0, \Omega}=\left(\sum_{E \in \mathcal{E}_{h}}\|w\|_{0, E}^{2}\right)^{\frac{1}{2}} \text { and }\|w\|_{s, \Omega}=\left(\sum_{E \in \mathcal{E}_{h}}\|w\|_{s, E}^{2}\right)^{\frac{1}{2}}, \quad s>0
$$

where $\|\cdot\|_{s, E}$ denotes the Sobolev $s$-norm over the element $E$.

We now recall some well-known facts that will be used in the error analysis in $§ 6-\S 9$. First, let us state some approximations properties and inequalities for the finite-element space. 
Lemma 2.1 (hp Approximation, [4, 5]) Let $E \in \mathcal{E}_{h}$ and $\psi \in H^{s}(E)$. Then there exist a positive constant $C$ independent of $\psi, r$, and $h$, and a sequence $\widetilde{\psi}_{r}^{h} \in \mathrm{P}_{r}(E), r=1,2, \ldots$, such that for any $q \in[0, s]$

$$
\begin{aligned}
& \left\|\psi-\widetilde{\psi}_{r}^{h}\right\|_{q, E} \leq C \frac{h^{\mu-q}}{r^{s-q}}\|\psi\|_{s, E}, \quad s \geq 0, \\
& \left\|\psi-\widetilde{\psi}_{r}^{h}\right\|_{0, e} \leq C \frac{h^{\mu-\frac{1}{2}}}{r^{s-\frac{1}{2}}}\|\psi\|_{s, E}, \quad s>\frac{1}{2},
\end{aligned}
$$

where $\mu:=\min (r+1, s)$ and $e$ is the edge on $\partial E$.

Lemma 2.2 (Trace Inequalities, [2]) Let $E \in \mathcal{E}_{h}$. Then for the trace operators $\gamma_{0}: H^{1}(E) \rightarrow$ $H^{\frac{1}{2}}(\partial E), \quad \gamma_{0} v=\left.v\right|_{\partial E}$ and $\gamma_{1}: H^{2}(E) \rightarrow H^{\frac{1}{2}}(\partial E), \quad \gamma_{1} v=\left.\frac{\partial v}{\partial n}\right|_{\partial E}$, there exists a constant $C_{t}$ independent of $h$ such that

$$
\begin{aligned}
& \forall w \in H^{s}(E), \quad s \geq 1, \quad\left\|\gamma_{0} w\right\|_{0, e} \leq C_{t} h^{-\frac{1}{2}}\left(\|w\|_{0, E}+h\|\nabla w\|_{0, E}\right), \\
& \forall w \in H^{s}(E), \quad s \geq 2, \quad\left\|\gamma_{1} w\right\|_{0, e} \leq C_{t} h^{-\frac{1}{2}}\left(\|\nabla w\|_{0, E}+h\left\|\nabla^{2} w\right\|_{0, E}\right),
\end{aligned}
$$

where e is the edge on $\partial E$.

Lemma 2.3 ([36]) Let $E$ be a mesh element with an edge e. Then there is a constant $C_{t}$ independent of $h$ and $r$ such that

$$
\begin{aligned}
& \|w\|_{0, e} \leq C_{t} h^{-\frac{1}{2}} r\|w\|_{0, E}, \\
& \left\|\nabla w \cdot \mathbf{n}_{e}\right\|_{0, e} \leq C_{t} h^{-\frac{1}{2}} r\|\nabla w\|_{0, E}, \quad \forall w \in \mathrm{P}_{r}(E)
\end{aligned}
$$

Lemma 2.4 ([3, 7]) There exists a constant $C$ independent of $h$ and $r$ such that

$$
\forall w \in \mathcal{W}_{r, h}\left(\mathcal{E}_{h}\right), \quad\|w\|_{0, \Omega}^{2} \leq C\left(\sum_{E \in \mathcal{E}_{h}}\|\nabla w\|_{0, E}^{2}+\sum_{e \in \Gamma_{h}} \frac{1}{|e|}\|[w]\|_{0, e}^{2}\right)^{\frac{1}{2}},
$$

where $|e|$ denotes the measure of $e$.

Lemma 2.5 (Inverse Inequalities, [37]) Let $E \in \mathcal{E}_{h}$ and $w \in \mathrm{P}_{r}(E)$. Then there exists a constant $C$ independent of $h$ and $r$ such that

$$
\begin{aligned}
& \|w\|_{L^{\infty}(E)} \leq C h^{-1} r^{2}\|w\|_{0, E} \\
& \|w\|_{1, E} \leq C h^{-1} r^{2}\|w\|_{0, E} .
\end{aligned}
$$

We also recall the following form of the discrete Gronwall's lemma:

Lemma 2.6 (discrete Gronwall) Let $\Delta t, H$, and $a_{n}, b_{n}, c_{n}, d_{n}$ (for integers $n \geq 0$ ) be nonnegative numbers such that $a_{l}+\Delta t \sum_{n=0}^{l} b_{n} \leq \Delta t \sum_{n=0}^{l} d_{n} a_{n}+\Delta t \sum_{n=0}^{l} c_{n}+H$ for $l \geq 0$. Suppose that $\Delta t d_{n}<1, \quad \forall n$. Then

$a_{l}+\Delta t \sum_{n=0}^{l} b_{n} \leq \exp \left(\Delta t \sum_{n=0}^{l} \frac{d_{n}}{1-\Delta t d_{n}}\right)\left(\Delta t \sum_{n=0}^{l} c_{n}+H\right)$ for $l \geq 0$. 
In the analysis below we also make the following assumptions:

- $\Omega$ is a rectangular domain with the boundary $\partial \Omega=\partial \Omega_{\text {ver }} \cup \partial \Omega_{\text {hor }}$, where $\partial \Omega_{\text {ver }}$ and $\partial \Omega_{\text {hor }}$ denote the vertical and horizontal pieces of the boundary $\partial \Omega$, respectively. We also split the set of interior edges, $\Gamma_{h}$, into two sets of vertical, $\Gamma_{h}^{\text {ver }}$, and horizontal, $\Gamma_{h}^{\text {hor }}$, edges, respectively;

- The degree of basis polynomials is $r \geq 2$ and the maximum diameter of the elements is $h<1$ (the latter assumption is only needed for simplification of the error analysis).

\section{Description of the Continuous in Time Numerical Scheme for the Keller-Segel Model}

We consider the Keller-Segel system (1.2). First, notice that the Jacobians of $\mathbf{F}$ and $\mathbf{G}$ are

$$
\frac{\partial \mathbf{F}}{\partial \mathbf{Q}}=\left(\begin{array}{cccc}
\chi u & 0 & \chi \rho & 0 \\
0 & 0 & 0 & 0 \\
0 & -1 & 0 & 0 \\
0 & 0 & 0 & 0
\end{array}\right) \quad \text { and } \quad \frac{\partial \mathbf{G}}{\partial \mathbf{Q}}=\left(\begin{array}{cccc}
\chi v & 0 & 0 & \chi \rho \\
0 & 0 & 0 & 0 \\
0 & 0 & 0 & 0 \\
0 & -1 & 0 & 0
\end{array}\right)
$$

and their eigenvalues are

$$
\lambda_{1}^{\mathbf{F}}=\chi u, \lambda_{2}^{\mathbf{F}}=\lambda_{3}^{\mathbf{F}}=\lambda_{4}^{\mathbf{F}}=0 \quad \text { and } \quad \lambda_{1}^{\mathbf{G}}=\chi v, \quad \lambda_{2}^{\mathbf{G}}=\lambda_{3}^{\mathbf{G}}=\lambda_{4}^{\mathbf{G}}=0,
$$

respectively. Hence, the convective part of (1.2) is hyperbolic. We now design semidiscrete interior penalty Galerkin methods for this system.

We assume that at any time level $t \in[0, T]$ the solution, $(\rho, c, u, v)^{T}$ is approximated by (discontinuous) piecewise polynomials of the corresponding degrees $r_{\rho}, r_{c}, r_{u}$, and $r_{v}$, which satisfy the following relation:

$$
\frac{r_{\max }}{r_{\min }} \leq a, \quad r_{\max }:=\max \left\{r_{\rho}, r_{c}, r_{u}, r_{v}\right\}, \quad r_{\min }:=\min \left\{r_{\rho}, r_{c}, r_{u}, r_{v}\right\}
$$

where $a$ is a constant independent of $r_{\rho}, r_{c}, r_{u}$, and $r_{v}$.

DG methods are formulated as follows. Find a continuous in time solution

$$
\left(\rho^{\mathrm{DG}}(\cdot, t), c^{\mathrm{DG}}(\cdot, t), u^{\mathrm{DG}}(\cdot, t), v^{\mathrm{DG}}(\cdot, t)\right) \in \mathcal{W}_{r_{\rho}, h}^{\rho} \times \mathcal{W}_{r_{c}, h}^{c} \times \mathcal{W}_{r_{u}, h}^{u} \times \mathcal{W}_{r_{v}, h}^{v},
$$

which satisfies the following weak formulation for the chemotaxis system (1.2):

$$
\begin{aligned}
& \int_{\Omega} \rho_{t}^{\mathrm{DG}} w^{\rho}+\sum_{E \in \mathcal{E}_{h}} \int_{E} \nabla \rho^{\mathrm{DG}} \nabla w^{\rho}-\sum_{e \in \Gamma_{h}} \int_{e}\left\{\nabla \rho^{\mathrm{DG}} \cdot \mathbf{n}_{e}\right\}\left[w^{\rho}\right]+\varepsilon \sum_{e \in \Gamma_{h}} \int_{e}\left\{\nabla w^{\rho} \cdot \mathbf{n}_{e}\right\}\left[\rho^{\mathrm{DG}}\right] \\
& +\sigma_{\rho} \sum_{e \in \Gamma_{h}} \frac{r_{\rho}^{2}}{|e|} \int_{e}\left[\rho^{\mathrm{DG}}\right]\left[w^{\rho}\right]-\sum_{E \in \mathcal{E}_{h}} \int_{E} \chi \rho^{\mathrm{DG}} u^{\mathrm{DG}}\left(w^{\rho}\right)_{x}+\sum_{e \in \Gamma_{h}^{\text {ver }}} \int_{e}\left(\chi \rho^{\mathrm{DG}} u^{\mathrm{DG}}\right)^{*} n_{x}\left[w^{\rho}\right] \\
& \quad-\sum_{E \in \mathcal{E}_{h}} \int_{E} \chi \rho^{\mathrm{DG}} v^{\mathrm{DG}}\left(w^{\rho}\right)_{y}+\sum_{e \in \Gamma_{h}^{\mathrm{hor}}} \int_{e}\left(\chi \rho^{\mathrm{DG}} v^{\mathrm{DG}}\right)^{*} n_{y}\left[w^{\rho}\right]=0
\end{aligned}
$$




$$
\begin{aligned}
& \int_{\Omega} c_{t}^{\mathrm{DG}} w^{c}+\sum_{E \in \mathcal{E}_{h}} \int_{E} \nabla c^{\mathrm{DG}} \nabla w^{c}-\sum_{e \in \Gamma_{h}} \int_{e}\left\{\nabla c^{\mathrm{DG}} \cdot \mathbf{n}_{e}\right\}\left[w^{c}\right]+\varepsilon \sum_{e \in \Gamma_{h}} \int_{e}\left\{\nabla w^{c} \cdot \mathbf{n}_{e}\right\}\left[c^{\mathrm{DG}}\right] \\
& +\sigma_{c} \sum_{e \in \Gamma_{h}} \frac{r_{c}^{2}}{|e|} \int_{e}\left[c^{\mathrm{DG}}\right]\left[w^{c}\right]+\int_{\Omega} c^{\mathrm{DG}} w^{c}-\int_{\Omega} \rho^{\mathrm{DG}} w^{c}=0, \\
& \int_{\Omega} u^{\mathrm{DG}} w^{u}+\sum_{E \in \mathcal{E}_{h}} \int_{E} c^{\mathrm{DG}}\left(w^{u}\right)_{x}+\sum_{e \in \Gamma_{h}^{\mathrm{ver}}} \int_{e}\left(-c^{\mathrm{DG}}\right)_{u}^{*} n_{x}\left[w^{u}\right] \\
& \quad-\sum_{e \in \partial \Omega_{\mathrm{ver}}} \int_{e} c^{\mathrm{DG}} n_{x} w^{u}+\sigma_{u} \sum_{e \in \Gamma_{h} \cup \partial \Omega_{\mathrm{ver}}} \frac{r_{u}^{2}}{|e|} \int_{e}\left[u^{\mathrm{DG}}\right]\left[w^{u}\right]=0, \\
& \int_{\Omega} v^{\mathrm{DG}} w^{v}+\sum_{E \in \mathcal{E}_{h}} \int_{E} c^{\mathrm{DG}}\left(w^{v}\right)_{y}+\sum_{e \in \Gamma_{h}^{\mathrm{hor}}} \int_{e}\left(-c^{\mathrm{DG}}\right)_{v}^{*} n_{y}\left[w^{v}\right] \\
& -\sum_{e \in \partial \Omega_{\mathrm{hor}}} \int_{e} c^{\mathrm{DG}} n_{y} w^{v}+\sigma_{v} \sum_{e \in \Gamma_{h} \cup \partial \Omega_{\mathrm{hor}}} \frac{r_{v}^{2}}{|e|} \int_{e}\left[v^{\mathrm{DG}}\right]\left[w^{v}\right]=0,
\end{aligned}
$$

and the initial conditions:

$$
\begin{aligned}
& \int_{\Omega} \rho^{\mathrm{DG}}(\cdot, 0) w^{\rho}=\int_{\Omega} \rho(\cdot, 0) w^{\rho}, \quad \int_{\Omega} c^{\mathrm{DG}}(\cdot, 0) w^{c}=\int_{\Omega} c(\cdot, 0) w^{c}, \\
& \int_{\Omega} u^{\mathrm{DG}}(\cdot, 0) w^{u}=\int_{\Omega} u(\cdot, 0) w^{u}, \quad \int_{\Omega} v^{\mathrm{DG}}(\cdot, 0) w^{v}=\int_{\Omega} v(\cdot, 0) w^{v} .
\end{aligned}
$$

Here, $\left(w^{\rho}, w^{c}, w^{u}, w^{v}\right) \in \mathcal{W}_{r_{\rho}, h}^{\rho} \times \mathcal{W}_{r_{c}, h}^{c} \times \mathcal{W}_{r_{u}, h}^{u} \times \mathcal{W}_{r_{v}, h}^{v}$ are the test functions, $\sigma_{\rho}, \sigma_{c}, \sigma_{u}$ and $\sigma_{v}$ are real positive penalty parameters. The parameter $\varepsilon$ is equal to either $-1,0$, or 1 : these values of $\varepsilon$ corresponding to the SIPG, IIPG, or NIPG method, respectively.

To approximate the convective terms in (3.3) and (3.5)-(3.6), we use the central-upwind fluxes from [30]:

$$
\begin{aligned}
& \left(\chi \rho^{\mathrm{DG}} u^{\mathrm{DG}}\right)^{*}=\frac{a^{\text {out }}\left(\chi \rho^{\mathrm{DG}} u^{\mathrm{DG}}\right)_{e}^{E^{1}}-a^{\text {in }}\left(\chi \rho^{\mathrm{DG}} u^{\mathrm{DG}}\right)_{e}^{E^{2}}}{a^{\text {out }}-a^{\text {in }}}-\frac{a^{\text {out }} a^{\text {in }}}{a^{\text {out }}-a^{\text {in }}}\left[\rho^{\mathrm{DG}}\right], \\
& \left(\chi \rho^{\mathrm{DG}} v^{\mathrm{DG}}\right)^{*}=\frac{b^{\text {out }}\left(\chi \rho^{\mathrm{DG}} v^{\mathrm{DG}}\right)_{e}^{E^{1}}-b^{\text {in }}\left(\chi \rho^{\mathrm{DG}} v^{\mathrm{DG}}\right)_{e}^{E^{2}}}{b^{\text {out }}-b^{\text {in }}}-\frac{b^{\text {out }} b^{\text {in }}}{b^{\text {out }}-b^{\text {in }}}\left[\rho^{\mathrm{DG}}\right], \\
& \left(-c^{\mathrm{DG}}\right)_{u}^{*}=-\frac{a^{\text {out }}\left(c^{\mathrm{DG}}\right)_{e}^{E^{1}}-a^{\text {in }}\left(c^{\mathrm{DG}}\right)_{e}^{E^{2}}}{a^{\text {out }}-a^{\text {in }}}-\frac{a^{\text {out }} a^{\text {in }}}{a^{\text {out }}-a^{\text {in }}}\left[u^{\mathrm{DG}}\right], \\
& \left(-c^{\mathrm{DG}}\right)_{v}^{*}=-\frac{b^{\text {out }}\left(c^{\mathrm{DG}}\right)_{e}^{E^{1}}-b^{\text {in }}\left(c^{\mathrm{DG}}\right)_{e}^{E^{2}}}{b^{\text {out }}-b^{\text {in }}}-\frac{b^{\text {out }} b^{\text {in }}}{b^{\text {out }}-b^{\text {in }}}\left[v^{\mathrm{DG}}\right] .
\end{aligned}
$$

Here, $a^{\text {out }}, a^{\text {in }}, b^{\text {out }}$, and $b^{\text {in }}$ are the one-sided local speeds in the $x$ - and $y$-directions. Since the convective part of the system (1.2) is hyperbolic, these speeds can be estimated using the largest and the smallest eigenvalues of the Jacobian $\frac{\partial \mathbf{F}}{\partial \mathbf{Q}}$ and $\frac{\partial \mathbf{G}}{\partial \mathbf{Q}}($ see $(3.1))$ :

$$
\begin{aligned}
& a^{\text {out }}=\max \left(\left(\chi u^{\mathrm{DG}}\right)_{e}^{E^{1}},\left(\chi u^{\mathrm{DG}}\right)_{e}^{E^{2}}, 0\right), \quad a^{\mathrm{in}}=\min \left(\left(\chi u^{\mathrm{DG}}\right)_{e}^{E^{1}},\left(\chi u^{\mathrm{DG}}\right)_{e}^{E^{2}}, 0\right), \\
& b^{\text {out }}=\max \left(\left(\chi v^{\mathrm{DG}}\right)_{e}^{E^{1}},\left(\chi v^{\mathrm{DG}}\right)_{e}^{E^{2}}, 0\right), \quad b^{\text {in }}=\min \left(\left(\chi v^{\mathrm{DG}}\right)_{e}^{E^{1}},\left(\chi v^{\mathrm{DG}}\right)_{e}^{E^{2}}, 0\right) .
\end{aligned}
$$


Remark. If $a^{\text {out }}-a^{\text {in }}=0$ at a certain element edge $e$, we set

$$
\begin{aligned}
& \left(\chi \rho^{\mathrm{DG}} u^{\mathrm{DG}}\right)^{*}=\frac{\left(\chi \rho^{\mathrm{DG}} u^{\mathrm{DG}}\right)_{e}^{E^{1}}+\left(\chi \rho^{\mathrm{DG}} u^{\mathrm{DG}}\right)_{e}^{E^{2}}}{2}, \quad\left(\chi \rho^{\mathrm{DG}} v^{\mathrm{DG}}\right)^{*}=\frac{\left(\chi \rho^{\mathrm{DG}} v^{\mathrm{DG}}\right)_{e}^{E^{1}}+\left(\chi \rho^{\mathrm{DG}} v^{\mathrm{DG}}\right)_{e}^{E^{2}}}{2} \\
& \left(-c^{\mathrm{DG}}\right)_{u}^{*}=-\frac{\left(c^{\mathrm{DG}}\right)_{e}^{E^{1}}+\left(c^{\mathrm{DG}}\right)_{e}^{E^{2}}}{2}, \quad\left(-c^{\mathrm{DG}}\right)_{v}^{*}=-\frac{\left(c^{\mathrm{DG}}\right)_{e}^{E^{1}}+\left(c^{\mathrm{DG}}\right)_{e}^{E^{2}}}{2}
\end{aligned}
$$

there. Notice that in any case, the following inequalities,

$$
\frac{a^{\text {out }}}{a^{\text {out }}-a^{\text {in }}} \leq 1, \quad \frac{-a^{\text {in }}}{a^{\text {out }}-a^{\text {in }}} \leq 1, \quad \frac{b^{\text {out }}}{b^{\text {out }}-b^{\text {in }}} \leq 1, \quad \text { and } \quad \frac{-b^{\text {in }}}{b^{\text {out }}-b^{\text {in }}} \leq 1,
$$

are satisfied.

From now on we will assume that $a^{\text {out }}-a^{\text {in }}>0$ and $b^{\text {out }}-b^{\text {in }}>0$ throughout the computational domain.

\section{Results for the Continuous in Time Scheme}

Let us recall here the results that were obtained in [18] for the continuous in time scheme (3.3)(3.6).

Lemma 4.1 (Consistency Lemma) If the solution of the system (1.2) belongs to $H^{2}\left(\mathcal{E}_{h}\right)$, then it satisfies the formulation (3.3)-(3.6).

Theorem $4.2\left(L^{2}\left(H^{1}\right)\right.$ and $L^{\infty}\left(L^{2}\right)$ error estimates). Let the solution $\rho, c, u$ and $v$ of the Keller-Segel system (1.2) be sufficiently regular. Furthermore, we assume that penalty parameters $\sigma_{\rho}, \sigma_{c}, \sigma_{u}, \sigma_{v}$ are sufficiently large. Then there exists at least one DG solution to (3.3)-(3.6) and there exists constants $C_{\rho}$ and $C_{c}$, independent of $h$ and $r$, such that

$$
\begin{aligned}
& \| \rho^{D G}- \rho\left\|_{L^{\infty}\left([0, T] ; L^{2}(\Omega)\right)}+\right\|\left|\nabla\left(\rho^{D G}-\rho\right) \|\right|_{L^{2}\left([0, T] ; L^{2}(\Omega)\right)}+\left(\int_{0}^{T} \sum_{e \in \Gamma_{h}} \frac{r_{\rho}^{2}}{|e|}\left\|\left[\rho^{D G}-\rho\right]\right\|_{0, e}^{2}\right)^{\frac{1}{2}} \\
& \leq C_{\rho}\left(\frac{h^{\min \left(r_{\rho}+1, s_{\rho}\right)-1}}{r_{\rho}^{s_{\rho}-2}}+\frac{h^{\min \left(r_{c}+1, s_{c}\right)-1}}{r_{c}^{s_{c}-2}}+\frac{h^{\min \left(r_{u}+1, s_{u}\right)-1}}{r_{u}^{s_{u}-2}}+\frac{h^{\min \left(r_{v}+1, s_{v}\right)-1}}{r_{v}^{s_{v}-2}}\right) \\
& \| c^{D G}- c\left\|_{L^{\infty}\left([0, T] ; L^{2}(\Omega)\right)}+\right\| \nabla\left(c^{D G}-c\right) \|\left.\right|_{L^{2}\left([0, T] ; L^{2}(\Omega)\right)}+\left(\int_{0}^{T} \sum_{e \in \Gamma_{h}} \frac{r_{c}^{2}}{|e|}\left\|\left[c^{D G}-c\right]\right\|_{0, e}^{2}\right)^{\frac{1}{2}} \\
& \leq C_{c}\left(\frac{h^{\min \left(r_{\rho}+1, s_{\rho}\right)-1}}{r_{\rho}^{s_{\rho}-2}}+\frac{h^{\min \left(r_{c}+1, s_{c}\right)-1}}{r_{c}^{s_{c}-2}}+\frac{h^{\min \left(r_{u}+1, s_{u}\right)-1}}{r_{u}^{s_{u}-2}}+\frac{h^{\min \left(r_{v}+1, s_{v}\right)-1}}{r_{v}^{s_{v}-2}}\right),
\end{aligned}
$$

where $\left(r_{\rho}, r_{c}, r_{u}, r_{v}\right) \geq 2$. 


\section{$5 \quad$ Fully Discrete Schemes and Analysis}

In the following sections, we formulate two explicit schemes and establish the convergence of the numerical solutions using an induction hypothesis. Existence of the discrete solution is trivial since the scheme is explicit in time. In the analysis below, we will assume that the exact solution of the system (1.2) is sufficiently regular for $t \leq T$, where $T$ is a pre-blow-up time. In particular, we will assume that

$$
(\rho, c, u, v) \in H^{s_{1}}([0, T]) \cap H^{s_{2}}(\Omega), \quad s_{1}>3 / 2, \quad s_{2} \geq 3,
$$

which is needed for the $h$-analysis (convergence rate with respect to the mesh size), or

$$
(\rho, c, u, v) \in H^{s_{1}}([0, T]) \cap H^{s_{2}}(\Omega), \quad s_{1}>3 / 2, \quad s_{2} \geq 5.5
$$

which is needed for the $r$-analysis (convergence rate with respect to the polynomial degree). Notice that these assumptions are reasonable since classical solutions of the Keller-Segel system (1.1) are regular (before the blow-up time) provided the initial data are sufficiently smooth, see [24] and references therein.

Let $\Delta t$ be a positive time step and let $t^{i}=i \Delta t$ denote the time step at the $i^{t h}$ step. We denote by $v^{i}$ the function $v$ evaluated at time $t^{i}$.

\section{Forward Euler Time Discretization}

Find a discrete in time solution

$$
\left(\rho_{\mathrm{DG}}^{i+1}, c_{\mathrm{DG}}^{i+1}, u_{\mathrm{DG}}^{i+1}, v_{\mathrm{DG}}^{i+1}\right) \in \mathcal{W}_{r_{\rho}, h}^{\rho} \times \mathcal{W}_{r_{c}, h}^{c} \times \mathcal{W}_{r_{u}, h}^{u} \times \mathcal{W}_{r_{v}, h}^{v},
$$

which satisfies the following weak formulation for the chemotaxis system (1.2):

$$
\begin{aligned}
& \int_{\Omega} \frac{\rho_{\mathrm{DG}}^{i+1}-\rho_{\mathrm{DG}}^{i}}{\Delta t} w^{\rho}+\sum_{E \in \mathcal{E}_{h}} \int_{E} \nabla \rho_{\mathrm{DG}}^{i} \nabla w^{\rho}-\sum_{e \in \Gamma_{h}} \int_{e}\left\{\nabla \rho_{\mathrm{DG}}^{i} \cdot \mathbf{n}_{e}\right\}\left[w^{\rho}\right]+\varepsilon \sum_{e \in \Gamma_{h}} \int_{e}\left\{\nabla w^{\rho} \cdot \mathbf{n}_{e}\right\}\left[\rho_{\mathrm{DG}}^{i}\right] \\
& +\sigma_{\rho} \sum_{e \in \Gamma_{h}} \frac{r_{\rho}^{2}}{|e|} \int_{e}\left[\rho_{\mathrm{DG}}^{i}\right]\left[w^{\rho}\right]-\sum_{E \in \mathcal{E}_{h}} \int_{E} \chi \rho_{\mathrm{DG}}^{i} u_{\mathrm{DG}}^{i}\left(w^{\rho}\right)_{x}+\sum_{e \in \Gamma_{h}^{\mathrm{ver}}} \int_{e}\left(\chi \rho_{\mathrm{DG}}^{i} u_{\mathrm{DG}}^{i}\right)^{*} n_{x}\left[w^{\rho}\right] \\
& \quad-\sum_{E \in \mathcal{E}_{h}} \int_{E} \chi \rho_{\mathrm{DG}}^{i} v_{\mathrm{DG}}^{i}\left(w^{\rho}\right)_{y}+\sum_{e \in \Gamma_{h}^{\mathrm{hor}}} \int_{e}\left(\chi \rho_{\mathrm{DG}}^{i} v_{\mathrm{DG}}^{i}\right)^{*} n_{y}\left[w^{\rho}\right]=0 \\
& \int_{\Omega} \frac{c_{\mathrm{DG}}^{i+1}-c_{\mathrm{DG}}^{i}}{\Delta t} w^{c}+\sum_{E \in \mathcal{E}_{h}} \int_{E} \nabla c_{\mathrm{DG}}^{i} \nabla w^{c}-\sum_{e \in \Gamma_{h}} \int_{e}\left\{\nabla c_{\mathrm{DG}}^{i} \cdot \mathbf{n}_{e}\right\}\left[w^{c}\right]+\varepsilon \sum_{e \in \Gamma_{h}} \int_{e}\left\{\nabla w^{c} \cdot \mathbf{n}_{e}\right\}\left[c_{\mathrm{DG}}^{i}\right] \\
& \quad+\sigma_{c} \sum_{e \in \Gamma_{h}} \frac{r_{c}^{2}}{|e|} \int_{e}\left[c_{\mathrm{DG}}^{i}\right]\left[w^{c}\right]+\int_{\Omega} c_{\mathrm{DG}}^{i} w^{c}-\int_{\Omega} \rho_{\mathrm{DG}}^{i} w^{c}=0 \\
& \int_{\Omega} u_{\mathrm{DG}}^{i+1} w^{u}+\sum_{E \in \mathcal{E}_{h}} \int_{E} c_{\mathrm{DG}}^{i+1}\left(w^{u}\right)_{x}+\sum_{e \in \Gamma_{h}^{\mathrm{ver}}} \int_{e}\left(-c_{\mathrm{DG}}^{i+1}\right)_{u}^{*} n_{x}\left[w^{u}\right]
\end{aligned}
$$




$$
\begin{gathered}
-\sum_{e \in \partial \Omega_{\mathrm{ver}}} \int_{e} c_{\mathrm{DG}}^{i+1} n_{x} w^{u}+\sigma_{u} \sum_{e \in \Gamma_{h} \cup \partial \Omega_{\mathrm{ver}}} \frac{r_{u}^{2}}{|e|} \int_{e}\left[u_{\mathrm{DG}}^{i+1}\right]\left[w^{u}\right]=0, \\
\int_{\Omega} v_{\mathrm{DG}}^{i+1} w^{v}+\sum_{E \in \mathcal{E}_{h}} \int_{E} c_{\mathrm{DG}}^{i+1}\left(w^{v}\right)_{y}+\sum_{e \in \Gamma_{h}^{\mathrm{hor}}} \int_{e}\left(-c_{\mathrm{DG}}^{i+1}\right)_{v}^{*} n_{y}\left[w^{v}\right] \\
-\sum_{e \in \partial \Omega_{\mathrm{hor}}} \int_{e} c_{\mathrm{DG}}^{i+1} n_{y} w^{v}+\sigma_{v} \sum_{e \in \Gamma_{h} \cup \partial \Omega_{\mathrm{hor}}} \frac{r_{v}^{2}}{|e|} \int_{e}\left[v_{\mathrm{DG}}^{i+1}\right]\left[w^{v}\right]=0 .
\end{gathered}
$$

To approximate the convective terms in (6.3), (6.5)-(6.6) we use the same central-upwind fluxes (3.8) as for the continuous in time scheme, with the one-sided local speeds given by:

$$
\begin{aligned}
& a_{i}^{\text {out }}:=\max \left(\left(\chi u_{\mathrm{DG}}^{i}\right)_{e}^{E^{1}},\left(\chi u_{\mathrm{DG}}^{i}\right)_{e}^{E^{2}}, 0\right), \quad a_{i}^{\text {in }}:=\min \left(\left(\chi u_{\mathrm{DG}}^{i}\right)_{e}^{E^{1}},\left(\chi u_{\mathrm{DG}}^{i}\right)_{e}^{E^{2}}, 0\right), \\
& b_{i}^{\text {out }}:=\max \left(\left(\chi v_{\mathrm{DG}}^{i}\right)_{e}^{E^{1}},\left(\chi v_{\mathrm{DG}}^{i}\right)_{e}^{E^{2}}, 0\right), \quad b_{i}^{\text {in }}:=\min \left(\left(\chi v_{\mathrm{DG}}^{i}\right)_{e}^{E^{1}},\left(\chi v_{\mathrm{DG}}^{i}\right)_{e}^{E^{2}}, 0\right) .
\end{aligned}
$$

Notice that the inequalities similar to (3.10),

$$
\frac{a_{i}^{\text {out }}}{a_{i}^{\text {out }}-a_{i}^{\text {in }}} \leq 1, \quad \frac{-a_{i}^{\text {in }}}{a_{i}^{\text {out }}-a_{i}^{\text {in }}} \leq 1, \quad \frac{b_{i}^{\text {out }}}{b_{i}^{\text {out }}-b_{i}^{\text {in }}} \leq 1, \quad \text { and } \quad \frac{-b_{i}^{\text {in }}}{b_{i}^{\text {out }}-b_{i}^{\text {in }}} \leq 1,
$$

which are needed in our convergence proof, are satisfied for the local speeds defined in (6.7) as well (for simplicity, we assume that $a_{i}^{\text {out }}-a_{i}^{\text {in }} \neq 0$ and $b_{i}^{\text {out }}-b_{i}^{\text {in }} \neq 0$ throughout the computational domain, see Remark in Section 3). Also, the initial conditions are:

$$
\begin{aligned}
& \int_{\Omega} \rho_{\mathrm{DG}}^{0} w^{\rho}=\int_{\Omega} \rho^{0} w^{\rho}, \quad \int_{\Omega} c_{\mathrm{DG}}^{0} w^{c}=\int_{\Omega} c^{0} w^{c}, \\
& \int_{\Omega} u_{\mathrm{DG}}^{0} w^{u}=\int_{\Omega} u^{0} w^{u}, \quad \int_{\Omega} v_{\mathrm{DG}}^{0} w^{v}=\int_{\Omega} v^{0} w^{v} .
\end{aligned}
$$

We denote by $\widetilde{\rho}^{i}, \widetilde{c}^{i}, \widetilde{u}^{i}$, and $\widetilde{v}^{i}$ the piecewise polynomial interpolants of the exact solution components $\rho^{i}, c^{i}, u^{i}$, and $v^{i}$ of the Keller-Segel system (1.2) and assume that these interpolants satisfy the approximation property (2.2). We then make the following induction hypothesis: assume that $\forall \quad i: 0 \leq i \leq n$, we have

$$
\begin{aligned}
S=\{ & \left(u_{\mathrm{DG}}^{i}, v_{\mathrm{DG}}^{i}\right) \in \mathcal{W}_{r_{u}, h}^{u} \times \mathcal{W}_{r_{v}, h}^{v}: \\
& \sum_{i=0}^{n} \Delta t\left\|u_{\mathrm{DG}}^{i}-\widetilde{u}^{i}\right\|_{0, \Omega}^{2} \\
& \leq C_{u}\left(\frac{h^{2 \min \left(r_{\rho}+1, s_{\rho}\right)-2}}{r_{\rho}^{2 s_{\rho}-3}}+\frac{h^{2 \min \left(r_{c}+1, s_{c}\right)-2}}{r_{c}^{2 s_{c}-3}}+\frac{h^{2 \min \left(r_{u}+1, s_{u}\right)-2}}{r_{u}^{2 s_{u}-3}}+\frac{h^{2 \min \left(r_{v}+1, s_{v}\right)-2}}{r_{v}^{2 s_{v}-3}}\right) \\
& +C_{u}^{t} \Delta t^{2}, \\
& \sum_{i=0}^{n} \Delta t\left\|v_{\mathrm{DG}}^{i}-\widetilde{v}^{i}\right\|_{0, \Omega}^{2}
\end{aligned}
$$




$$
\begin{aligned}
& \leq C_{v}\left(\frac{h^{2 \min \left(r_{\rho}+1, s_{\rho}\right)-2}}{r_{\rho}^{2 s_{\rho}-3}}+\frac{h^{2 \min \left(r_{c}+1, s_{c}\right)-2}}{r_{c}^{2 s_{c}-3}}+\frac{h^{2 \min \left(r_{u}+1, s_{u}\right)-2}}{r_{u}^{2 s_{u}-3}}+\frac{h^{2 \min \left(r_{v}+1, s_{v}\right)-2}}{r_{v}^{2 s_{v}-3}}\right) \\
& +C_{v}^{t} \Delta t^{2}, \\
& \left.\sup _{0 \leq i \leq n}\left\|u_{\mathrm{DG}}^{i}-\widetilde{u}^{i}\right\|_{0, \Omega} \leq C_{u}^{\star} \frac{h}{r_{\min }^{2}}, \quad \sup _{0 \leq i \leq n}\left\|v_{\mathrm{DG}}^{i}-\widetilde{v}^{i}\right\|_{0, \Omega} \leq C_{v}^{\star} \frac{h}{r_{\text {min }}^{2}}\right\},
\end{aligned}
$$

where $C_{u}, C_{v}, C_{u}^{t}, C_{v}^{t}, C_{u}^{\star}$, and $C_{v}^{\star}$ are positive constants (which will be defined later) independent of $h$, the polynomial degrees $\left(r_{\rho}, r_{c}, r_{u}, r_{v}\right)$, and $n$. The parameters $s_{\rho}, s_{c}, s_{u}$, and $s_{v}$ denote the regularity of the corresponding components of the exact solution. Clearly, the induction hypothesis above holds true for $i=0$. We need now to show that assuming that $S$ holds true $\forall \quad i: 0 \leq i \leq n$, it will follow that it will be true for $i+1=n+1$.

Let us first show that from the induction hypothesis $S$, it follows that functions $u_{\mathrm{DG}}^{i}$, and $v_{\mathrm{DG}}^{i}, 1 \leq$ $i \leq n$ are bounded.

Lemma 6.1 For $\left(u_{\mathrm{DG}}^{i}, v_{\mathrm{DG}}^{i}\right) \in S$, there exist positive constants $M_{u}$, and $M_{v}$ independent of $h, r_{u}$, and $r_{v}$, such that

$$
\sup _{0 \leq i \leq n}\left\|u_{\mathrm{DG}}^{i}\right\|_{\infty, \Omega} \leq M_{u}, \quad \sup _{0 \leq i \leq n}\left\|v_{\mathrm{DG}}^{i}\right\|_{\infty, \Omega} \leq M_{v}
$$

Proof: The result is derived from the definition of the subset $S$ and the inverse inequality .

Theorem $6.2\left(l^{2}\left(H^{1}\right)\right.$ and $l^{\infty}\left(L^{2}\right)$ Forward Euler error estimates $)$. Let the solution $\rho, c, u$ and $v$ of the Keller-Segel system (1.2) be sufficiently regular. Furthermore, we assume that penalty parameters $\sigma_{\rho}, \sigma_{c}, \sigma_{u}, \sigma_{v}$ are sufficiently large. Then the induction hypothesis holds true for $n+1$. Furthermore, there exists constants $C_{\rho}$ and $C_{c}$, independent of $h$ and $r$, such that

$$
\begin{gathered}
\left\|\rho_{D G}-\rho\right\|_{l^{\infty}\left([0, T] ; L^{2}(\Omega)\right)}+\Delta t^{\frac{1}{2}}\left\|\mid \nabla\left(\rho_{D G}-\rho\right)\right\| \|_{l^{2}\left([0, T] ; L^{2}(\Omega)\right)}+\left(\sum_{i=0}^{N} \Delta t \sum_{e \in \Gamma_{h}} \frac{r_{\rho}^{2}}{|e|}\left\|\left[\rho_{D G}^{i}-\rho^{i}\right]\right\|_{0, e}^{2}\right)^{\frac{1}{2}} \\
\leq C_{\rho}\left(\frac{h^{\min \left(r_{\rho}+1, s_{\rho}\right)-1}}{r_{\rho}^{s_{\rho}-\frac{3}{2}}}+\frac{h^{\min \left(r_{c}+1, s_{c}\right)-1}}{r_{c}^{s_{c}-\frac{3}{2}}}+\frac{h^{\min \left(r_{u}+1, s_{u}\right)-1}}{r_{u}^{s_{u}-\frac{3}{2}}}+\frac{h^{\min \left(r_{v}+1, s_{v}\right)-1}}{r_{v}^{s_{v}-\frac{3}{2}}}+\Delta t\right) \\
\left\|c_{D G}-c\right\|_{l^{\infty}\left([0, T] ; L^{2}(\Omega)\right)}+\Delta t^{\frac{1}{2}} \mid\left\|\nabla\left(c_{D G}-c\right)\right\| \|_{l^{2}\left([0, T] ; L^{2}(\Omega)\right)}+\left(\sum_{i=0}^{N} \Delta t \sum_{e \in \Gamma_{h}} \frac{r_{c}^{2}}{|e|}\left\|\left[c_{D G}^{i}-c^{i}\right]\right\|_{0, e}^{2}\right)^{\frac{1}{2}} \\
\leq C_{c}\left(\frac{h^{\min \left(r_{\rho}+1, s_{\rho}\right)-1}}{r_{\rho}^{s_{\rho}-\frac{3}{2}}}+\frac{h^{\min \left(r_{c}+1, s_{c}\right)-1}}{r_{c}^{s_{c}-\frac{3}{2}}}+\frac{h^{\min \left(r_{u}+1, s_{u}\right)-1}}{r_{u}^{s_{u}-\frac{3}{2}}}+\frac{h^{\min \left(r_{v}+1, s_{v}\right)-1}}{r_{v}^{s_{v}-\frac{3}{2}}}+\Delta t\right),
\end{gathered}
$$

where $\left(r_{\rho}, r_{c}, r_{u}, r_{v}\right) \geq 2$.

Proof: We introduce the following notation:

$$
\begin{aligned}
& \tau_{\rho}^{i}:=\rho_{\mathrm{DG}}^{i}-\widetilde{\rho}^{i}, \quad \xi_{\rho}^{i}:=\rho^{i}-\widetilde{\rho}^{i}, \quad \tau_{c}^{i}:=c_{\mathrm{DG}}^{i}-\widetilde{c}^{i}, \quad \xi_{c}^{i}:=c^{i}-\widetilde{c}^{i}, \\
& \tau_{u}^{i}:=u_{\mathrm{DG}}^{i}-\widetilde{u}^{i}, \quad \xi_{u}^{i}=u^{i}-\widetilde{u}^{i}, \quad \tau_{v}^{i}:=v_{\mathrm{DG}}^{i}-\widetilde{v}^{i}, \quad \xi_{v}^{i}:=v^{i}-\widetilde{v}^{i} .
\end{aligned}
$$


It follows from the consistency Lemma 4.1(see [18] for the details) that the exact solution of (1.2) satisfies the following weak formulation:

$$
\begin{gathered}
\int_{\Omega} \rho_{t} w^{\rho}+\sum_{E \in \mathcal{E}_{h}} \int_{E} \nabla \rho \nabla w^{\rho}-\sum_{e \in \Gamma_{h}} \int_{e}\left\{\nabla \rho \cdot \mathbf{n}_{e}\right\}\left[w^{\rho}\right]+\varepsilon \sum_{e \in \Gamma_{h}} \int_{e}\left\{\nabla w^{\rho} \cdot \mathbf{n}_{e}\right\}[\rho]+\sigma_{\rho} \sum_{e \in \Gamma_{h}} \frac{r_{\rho}^{2}}{|e|} \int_{e}[\rho]\left[w^{\rho}\right] \\
-\sum_{E \in \mathcal{E}_{h}} \int_{E} \chi \rho u\left(w^{\rho}\right)_{x}+\sum_{e \in \Gamma_{h}^{\text {ver }}} \int_{e}(\chi \rho u)^{* *} n_{x}\left[w^{\rho}\right]-\sum_{E \in \mathcal{E}_{h}} \int_{E} \chi \rho v\left(w^{\rho}\right)_{y}+\sum_{e \in \Gamma_{h}^{\text {hor }}} \int_{e}(\chi \rho v)^{* *} n_{y}\left[w^{\rho}\right]=0,(6.15)
\end{gathered}
$$

where

$$
\begin{aligned}
(\chi \rho u)^{* *} & :=\frac{a_{i}^{\text {out }}(\chi \rho u)_{e}^{E^{1}}-a_{i}^{\text {in }}(\chi \rho u)_{e}^{E^{2}}}{a_{i}^{\text {out }}-a_{i}^{\text {in }}}-\frac{a_{i}^{\text {out }} a_{i}^{\text {in }}}{a_{i}^{\text {out }}-a_{i}^{\text {in }}}[\rho], \\
(\chi \rho v)^{* *}: & :=\frac{b_{i}^{\text {out }}(\chi \rho v)_{e}^{E^{1}}-b_{i}^{\text {in }}(\chi \rho v)_{e}^{E^{2}}}{b_{i}^{\text {out }}-b_{i}^{\text {in }}}-\frac{b_{i}^{\text {out }} b_{i}^{\text {in }}}{b_{i}^{\text {out }}-b_{i}^{\text {in }}}[\rho],
\end{aligned}
$$

and the local speeds $a_{i}^{\text {out }}, a_{i}^{\text {in }}, b_{i}^{\text {out }}$, and $b_{i}^{\text {in }}$ are given by (6.7). Using (6.14), equation (6.15) can be rewritten as:

$$
\begin{aligned}
& \int_{\Omega} \widetilde{\rho}_{t}\left(t^{i}\right) w^{\rho}+\sum_{E \in \mathcal{E}_{h}} \int_{E} \nabla \widetilde{\rho}^{i} \nabla w^{\rho}-\sum_{e \in \Gamma_{h}} \int_{e}\left\{\nabla \widetilde{\rho}^{i} \cdot \mathbf{n}_{e}\right\}\left[w^{\rho}\right]+\varepsilon \sum_{e \in \Gamma_{h}} \int_{e}\left\{\nabla w^{\rho} \cdot \mathbf{n}_{e}\right\}\left[\tilde{\rho}^{i}\right]+\sigma_{\rho} \sum_{e \in \Gamma_{h}} \frac{r_{\rho}^{2}}{|e|} \int_{e}\left[\widetilde{\rho}^{i}\right]\left[w^{\rho}\right] \\
&- \sum_{E \in \mathcal{E}_{h}} \int_{E} \chi \widetilde{\rho}^{i} u_{\mathrm{DG}}^{i}\left(w^{\rho}\right)_{x}+\sum_{E \in \mathcal{E}_{h}} \int_{E} \chi \widetilde{\rho}^{i} \tau_{u}^{i}\left(w^{\rho}\right)_{x}-\sum_{E \in \mathcal{E}_{h}} \int_{E} \chi \widetilde{\rho}^{i} \xi_{u}^{i}\left(w^{\rho}\right)_{x}+\sum_{e \in \Gamma_{h}^{\text {ver }}} \int_{e}\left(\chi \rho^{i} u^{i}\right)^{* *} n_{x}\left[w^{\rho}\right] \\
&-\sum_{E \in \mathcal{E}_{h}} \int_{E} \chi \widetilde{\rho}^{i} v_{\mathrm{DG}}^{i}\left(w^{\rho}\right)_{y}+\sum_{E \in \mathcal{E}_{h}} \int_{E} \chi \widetilde{\rho}^{i} \tau_{v}^{i}\left(w^{\rho}\right)_{x}-\sum_{E \in \mathcal{E}_{h}} \int_{E} \chi \widetilde{\rho}^{i} \xi_{v}^{i}\left(w^{\rho}\right)_{x}+\sum_{e \in \Gamma_{h}^{\mathrm{hor}}} \int_{e}\left(\chi \rho^{i} v^{i}\right)^{* *} n_{y}\left[w^{\rho}\right] \\
&=-\int_{\Omega} \xi_{\rho t}\left(t^{i}\right) w^{\rho}-\sum_{E \in \mathcal{E}_{h}} \int_{E} \nabla \xi_{\rho}^{i} \nabla w^{\rho}+\sum_{e \in \Gamma_{h}} \int_{e}\left\{\nabla \xi_{\rho}^{i} \cdot \mathbf{n}_{e}\right\}\left[w^{\rho}\right]-\varepsilon \sum_{e \in \Gamma_{h}} \int_{e}\left\{\nabla w^{\rho} \cdot \mathbf{n}_{e}\right\}\left[\xi_{\rho}^{i}\right] \\
&-\sigma_{\rho} \sum_{e \in \Gamma_{h}} \frac{r_{\rho}^{2}}{|e|} \int_{e}\left[\xi_{\rho}^{i}\right]\left[w^{\rho}\right]+\sum_{E \in \mathcal{E}_{h}} \int_{E} \chi \xi_{\rho}^{i} u^{i}\left(w^{\rho}\right)_{x}+\sum_{E \in \mathcal{E}_{h}} \int_{E} \chi \xi_{\rho}^{i} v^{i}\left(w^{\rho}\right)_{y}
\end{aligned}
$$

In order to obtain the estimate on the time step $\Delta t=O\left(\frac{h^{2}}{r^{4}}\right)$ we will proceed as follows, subtract equation (6.16) from (6.3) and choose $w^{\rho}=\tau_{\rho}^{i}$, to obtain

$$
\begin{gathered}
\left\|\tau_{\rho}^{i+1}\right\|_{0, \Omega}^{2}-\left\|\tau_{\rho}^{i}\right\|_{0, \Omega}^{2}+2 \Delta t\left|\left\|\nabla \tau_{\rho}^{i}\right\|\right|_{0, \Omega}^{2}+2 \Delta t \sigma_{\rho} \sum_{e \in \Gamma_{h}} \frac{r_{\rho}^{2}}{|e|}\left\|\left[\tau_{\rho}^{i}\right]\right\|_{0, e}^{2}=\left\|\tau_{\rho}^{i+1}-\tau_{\rho}^{i}\right\|_{0, \Omega}^{2} \\
+(1-\varepsilon) 2 \Delta t \sum_{e \in \Gamma_{h}} \int_{e}\left\{\nabla \tau_{\rho}^{i} \cdot \mathbf{n}_{e}\right\}\left[\tau_{\rho}^{i}\right]+2 \Delta t \sum_{E \in \mathcal{E}_{h}} \int_{E} \chi \tau_{\rho}^{i} u_{\mathrm{DG}}^{i}\left(\tau_{\rho}^{i}\right)_{x}+2 \Delta t \sum_{E \in \mathcal{E}_{h}} \int_{E} \chi \widetilde{\rho}^{i} \tau_{u}^{i}\left(\tau_{\rho}^{i}\right)_{x}-2 \Delta t \sum_{E \in \mathcal{E}_{h}} \int_{E} \chi \widetilde{\rho}^{i} \xi_{u}^{i}\left(\tau_{\rho}^{i}\right)_{x} \\
-2 \Delta t \sum_{e \in \Gamma_{h}^{\text {ver }}} \int_{e}\left(\left(\chi \rho_{\mathrm{DG}}^{i} u_{\mathrm{DG}}^{i}\right)^{*}-\left(\chi \rho^{i} u^{i}\right)^{* *}\right) n_{x}\left[\tau_{\rho}^{i}\right]+2 \Delta t \sum_{E \in \mathcal{E}_{h}} \int_{E} \chi \tau_{\rho}^{i} v_{\mathrm{DG}}^{i}\left(\tau_{\rho}^{i}\right)_{y}+2 \Delta t \sum_{E \in \mathcal{E}_{h}} \int_{E} \chi \widetilde{\rho}^{i} \tau_{v}^{i}\left(\tau_{\rho}^{i}\right)_{y} \\
-2 \Delta t \sum_{E \in \mathcal{E}_{h}} \int_{E} \chi \widetilde{\rho}^{i} \xi_{v}^{i}\left(\tau_{\rho}^{i}\right)_{y}-2 \Delta t \sum_{e \in \Gamma_{h}^{\mathrm{hor}}} \int_{e}\left(\left(\chi \rho_{\mathrm{DG}}^{i} v_{\mathrm{DG}}^{i}\right)^{*}-\left(\chi \rho^{i} v^{i}\right)^{* *}\right) n_{y}\left[\tau_{\rho}^{i}\right]
\end{gathered}
$$




$$
\begin{gathered}
+2 \Delta t \int_{\Omega} \xi_{\rho_{t}}\left(t^{i}\right) \tau_{\rho}^{i}+2 \Delta t \int_{\Omega}\left(\widetilde{\rho}_{t}\left(t^{i}\right)-\frac{\widetilde{\rho}^{i+1}-\widetilde{\rho}^{i}}{\Delta t}\right) \tau_{\rho}^{i}+2 \Delta t \sum_{E \in \mathcal{E}_{h}} \int_{E} \nabla \xi_{\rho}^{i} \nabla \tau_{\rho}^{i}-2 \Delta t \sum_{e \in \Gamma_{h}} \int_{e}\left\{\nabla \xi_{\rho}^{i} \cdot \mathbf{n}_{e}\right\}\left[\tau_{\rho}^{i}\right] \\
+\varepsilon 2 \Delta t \sum_{e \in \Gamma_{h}} \int_{e}\left\{\nabla \tau_{\rho}^{i} \cdot \mathbf{n}_{e}\right\}\left[\xi_{\rho}^{i}\right]+2 \Delta t \sigma_{\rho} \sum_{e \in \Gamma_{h}} \frac{r_{\rho}^{2}}{|e|} \int_{e}\left[\xi_{\rho}^{i}\right]\left[\tau_{\rho}^{i}\right]-2 \Delta t \sum_{E \in \mathcal{E}_{h}} \int_{E} \chi \xi_{\rho}^{i} u^{i}\left(\tau_{\rho}^{i}\right)_{x}-2 \Delta t \sum_{E \in \mathcal{E}_{h}} \int_{E} \chi \xi_{\rho}^{i} v^{i}\left(\tau_{\rho}^{i}\right)_{y} \\
=: T_{1}^{\rho}+T_{2}^{\rho}+\ldots+T_{18}^{\rho} .
\end{gathered}
$$

Let us remark that term $\int_{\Omega} \frac{\tau_{\rho}^{i+1}-\tau_{\rho}^{i}}{\Delta t} \tau_{\rho}^{i}$ on the LHS of (6.17) was rewritten as:

$$
\int_{\Omega} \frac{\tau_{\rho}^{i+1}-\tau_{\rho}^{i}}{\Delta t} \tau_{\rho}^{i}=\frac{\left\|\tau_{\rho}^{i+1}\right\|_{0, \Omega}^{2}}{2 \Delta t}-\frac{\left\|\tau_{\rho}^{i}\right\|_{0, \Omega}^{2}}{2 \Delta t}-\frac{\left\|\tau_{\rho}^{i+1}-\tau_{\rho}^{i}\right\|_{0, \Omega}^{2}}{2 \Delta t}
$$

Next, we bound each term on the RHS of (6.17) starting from term $T_{2}^{\rho}$ using standard DG techniques, the term $T_{1}^{\rho}$ will be bounded last. The quantities $\varepsilon_{i}$ in the estimates below are positive real numbers, which will be defined later.

Consider now, second term on the RHS $T_{2}^{\rho}$ :

$$
\left|T_{2}^{\rho}\right| \leq 4 \Delta t \sum_{e \in \Gamma_{h}}\left\|\left\{\nabla \tau_{\rho}^{i}\right\}\right\|_{0, e}\left\|\left[\tau_{\rho}^{i}\right]\right\|_{0, e} .
$$

As before, we denote by $E^{1}$ and $E^{2}$ the two elements sharing the edge $e$. Then, using the inequality (2.6), we obtain

$$
\begin{aligned}
\Delta t \sum_{e \in \Gamma_{h}}\left\|\left\{\nabla \tau_{\rho}^{i}\right\}\right\|_{0, e}\left\|\left[\tau_{\rho}^{i}\right]\right\|_{0, e} & \leq 4 \Delta t \sum_{e \in \Gamma_{h}} \frac{1}{2}\left(\left\|\left(\nabla \tau_{\rho}^{i}\right)_{e}^{E^{1}}\right\|_{0, e}+\left\|\left(\nabla \tau_{\rho}^{i}\right)_{e}^{E^{2}}\right\|_{0, e}\right)\left\|\left[\tau_{\rho}^{i}\right]\right\|_{0, e} \\
& \leq \frac{2 \Delta t C_{t} r_{\rho}}{\sqrt{h}} \sum_{e \in \Gamma_{h}^{\text {ver }} \cup \Gamma_{h}^{\text {hor }}}\left(\left\|\nabla \tau_{\rho}^{i}\right\|_{0, E^{1}}+\left\|\nabla \tau_{\rho}^{i}\right\|_{0, E^{2}}\right)\left\|\left[\tau_{\rho}^{i}\right]\right\|_{0, e},
\end{aligned}
$$

and hence, using the fact that $|e| \leq \sqrt{h}$ and using Cauchy-Schwarz inequality, we end up with the following bound on $T_{2}^{\rho}$ :

$$
\left|T_{2}^{\rho}\right| \leq \varepsilon_{2}^{\rho} \Delta t\left|\left\|\nabla \tau_{\rho}^{i} \mid\right\|_{0, \Omega}^{2}+R_{2} \Delta t \sum_{e \in \Gamma_{h}} \frac{r_{\rho}^{2}}{|e|}\left\|\left[\tau_{\rho}^{i}\right]\right\|_{0, e}^{2}\right.
$$

The term $T_{3}^{\rho}$ is bounded using Lemma 6.1 for $u_{\mathrm{DG}}^{i}$ and using Cauchy-Schwarz and Young's inequality:

$$
\left|T_{3}^{\rho}\right| \leq \varepsilon_{3}^{\rho} \Delta t\left|\left\|\nabla \tau_{\rho}^{i} \mid\right\|_{0, \Omega}^{2}+R_{3} \Delta t\left\|\tau_{\rho}^{i}\right\|_{0, \Omega}^{2}\right.
$$

The term $T_{4}^{\rho}$ is bounded using Cauchy-Schwarz and Young's inequalities and assumption (3.2):

$$
\left|T_{4}^{\rho}\right| \leq \Delta t \sum_{E \in \mathcal{E}_{h}} K\left\|\nabla \tau_{\rho}^{i}\right\|_{0, E}\left\|\tau_{u}^{i}\right\|_{0, E} \leq \varepsilon_{4}^{\rho} \Delta t\left|\left\|\nabla \tau_{\rho}^{i} \mid\right\|_{0, \Omega}^{2}+R_{4} \Delta t\left\|\tau_{u}^{i}\right\|_{0, \Omega}^{2}\right.
$$

The term $T_{5}^{\rho}$ is bounded using Cauchy-Schwarz, Young's inequality and approximation results $(2.2)$ :

$$
\left|T_{5}^{\rho}\right| \leq\left.\varepsilon_{5}^{\rho} \Delta t||\left|\nabla \tau_{\rho}^{i}\right|\right|_{0, \Omega} ^{2}+R_{5} \Delta t \frac{h^{2 \min \left(r_{u}+1, s_{u}\right)}}{r_{u}^{2 s_{u}}}
$$


Next, we bound $T_{6}^{\rho}$ on the RHS of (6.17) as

$$
\begin{aligned}
\left|T_{6}^{\rho}\right| \leq 2 \Delta t \sum_{e \in \Gamma_{h}^{\text {ver }}} & \left(\left|\int_{e} \frac{a_{i}^{\text {out }}}{a_{i}^{\text {out }}-a_{i}^{\text {in }}}\left(\left(\chi \rho_{\mathrm{DG}}^{i} u_{\mathrm{DG}}^{i}\right)_{e}^{E^{1}}-\left(\chi \rho^{i} u^{i}\right)_{e}^{E^{1}}\right) n_{x}\left[\tau_{\rho}^{i}\right]\right|\right. \\
+ & \left|\int_{e} \frac{-a_{i}^{\text {in }}}{a_{i}^{\text {out }}-a_{i}^{\text {in }}}\left(\left(\chi \rho_{\mathrm{DG}}^{i} u_{\mathrm{DG}}^{i}\right)_{e}^{E^{2}}-\left(\chi \rho^{i} u^{i}\right)_{e}^{E^{2}}\right) n_{x}\left[\tau_{\rho}^{i}\right]\right| \\
+ & \left.\left|\int_{e} \frac{-a_{i}^{\text {in }} a_{i}^{\text {out }}}{a_{i}^{\text {out }}-a_{i}^{\text {in }}}\left[\rho_{\mathrm{DG}}^{i}-\rho^{i}\right] n_{x}\left[\tau_{\rho}^{i}\right]\right|\right)=: \mathrm{I}+\mathrm{II}+\mathrm{III} .
\end{aligned}
$$

Using (6.8) and (6.14), the first term on the RHS of (6.23) can be estimated by

$$
\begin{aligned}
& \mathrm{I} \leq 2 \Delta t \chi \sum_{e \in \Gamma_{h}^{\mathrm{ver}}}\left|\int_{e}\left(\left(\chi \rho_{\mathrm{DG}}^{i} u_{\mathrm{DG}}^{i}\right)_{e}^{E^{1}}-\left(\chi \rho^{i} u^{i}\right)_{e}^{E^{1}}\right) n_{x}\left[\tau_{\rho}^{i}\right]\right| \\
& \leq 2 \Delta t \chi \sum_{e \in \Gamma_{h}^{\mathrm{ver}}}\left(\left|\int_{e}\left(\tau_{\rho}^{i} u_{\mathrm{DG}}^{i}\right)_{e}^{E^{1}} n_{x}\left[\tau_{\rho}^{i}\right]\right|+\left|\int_{e}\left(\xi_{\rho}^{i} u_{\mathrm{DG}}^{i}\right)_{e}^{E^{1}} n_{x}\left[\tau_{\rho}^{i}\right]\right|\right. \\
& \left.+\left|\int_{e}\left(\rho^{i} \tau_{u}^{i}\right)_{e}^{E^{1}} n_{x}\left[\tau_{\rho}^{i}\right]\right|+\left|\int_{e}\left(\xi_{u}^{i} \rho^{i}\right)_{e}^{E^{1}} n_{x}\left[\tau_{\rho}^{i}\right]\right|\right)=: \widetilde{\mathrm{I}} .
\end{aligned}
$$

We now use the Cauchy-Schwarz inequality, the trace inequality (2.4), the inequality (2.6), the assumption (3.2), the assumption that $h<1, r>1, \Delta t<1$, the approximation inequality (2.2), the bound on $u_{\mathrm{DG}}^{i}$ from Lemma 6.1:

$\widetilde{\mathrm{I}} \leq \widetilde{\varepsilon_{1}} \Delta t\left\|\tau_{\rho}^{i}\right\|_{0, \Omega}^{2}+\left(K_{1}+K_{2}\right) \Delta t \sum_{e \in \Gamma_{h}} \frac{r_{\rho}^{2}}{|e|}\left\|\left[\tau_{\rho}^{i}\right]\right\|_{0, e}^{2}+K_{3} \Delta t\left\|\tau_{u}^{i}\right\|_{0, \Omega}^{2}+K_{4} \Delta t\left(\frac{h^{2 \min \left(r_{\rho}+1, s_{\rho}\right)}}{r_{\rho}^{2 s_{\rho}}}+\frac{h^{2 \min \left(r_{u}+1, s_{u}\right)}}{r_{u}^{2 s_{u}}}\right)$.

A similar bound can be derived for the second term II on the RHS of (6.23). To estimate the last term on the RHS of (6.23), we first use (6.14) and the definition of the one-sided local speeds (6.7) to obtain

$$
\mathrm{III} \leq C \Delta t \sum_{e \in \Gamma_{h}^{\text {ver }}}\left(\left\|\left[\tau_{\rho}^{i}\right]\right\|_{0, e}^{2}+\left|\int_{e}\left[\xi_{\rho}^{i}\right]\left[\tau_{\rho}^{i}\right]\right|\right):=\widetilde{\mathrm{III}}
$$

Then, using the Cauchy-Schwarz inequality, the assumption that $h<1$ (and choosing $h$ small enough), $r>1, \Delta t<1$, the approximation inequality (2.2), and inequality (2.4), we bound III as follows:

$$
\widetilde{\mathrm{III}} \leq \sum_{e \in \Gamma_{h}^{\text {ver }}} \frac{\Delta t r_{\rho}^{2}}{|e|}\left\|\left[\tau_{\rho}^{i}\right]\right\|_{0, e}^{2}+\Delta t \frac{h^{2 \min \left(r_{\rho}+1, s_{\rho}\right)-1}}{r_{\rho}^{2 s_{\rho}}} .
$$

Combining the above bounds on I, II, and III, we arrive at

$$
\left|T_{6}^{\rho}\right| \leq \varepsilon_{6}^{\rho} \Delta t\left\|\tau_{\rho}^{i}\right\|_{0, \Omega}^{2}+R_{6} \Delta t \sum_{e \in \Gamma_{h}} \frac{r_{\rho}^{2}}{|e|}\left\|\left[\tau_{\rho}^{i}\right]\right\|_{0, e}^{2}+R_{7} \Delta t\left\|\tau_{u}^{i}\right\|_{0, \Omega}^{2}+R_{8} \Delta t\left(\frac{h^{2 \min \left(r_{\rho}+1, s_{\rho}\right)-1}}{r_{\rho}^{2 s_{\rho}}}+\frac{h^{2 \min \left(r_{u}+1, s_{u}\right)}}{r_{u}^{2 s_{u}}}\right) .
$$


The terms $T_{7}^{\rho}-T_{10}^{\rho}$ are bounded in the same way as the terms $T_{3}^{\rho}-T_{6}^{\rho}$, respectively, and the bounds are:

$$
\begin{gathered}
\left|T_{7}^{\rho}\right| \leq \varepsilon_{7}^{\rho} \Delta t\left|\left\|\nabla \tau_{\rho}^{i} \mid\right\|_{0, \Omega}^{2}+R_{9} \Delta t\left\|\tau_{\rho}^{i}\right\|_{0, \Omega}^{2}\right. \\
\left|T_{8}^{\rho}\right| \leq \varepsilon_{8}^{\rho} \Delta t\left|\left\|\nabla \tau_{\rho}^{i} \mid\right\|_{0, \Omega}^{2}+R_{10} \Delta t\left\|\tau_{v}^{i}\right\|_{0, \Omega}^{2}\right. \\
\left|T_{9}^{\rho}\right| \leq\left.\varepsilon_{9}^{\rho} \Delta t\left|\| \nabla \tau_{\rho}^{i}\right|\right|_{0, \Omega} ^{2}+R_{11} \Delta t \frac{h^{2 \min \left(r_{v}+1, s_{v}\right)}}{r_{v}^{2 s_{v}}} \\
\left|T_{10}^{\rho}\right| \leq \varepsilon_{10}^{\rho} \Delta t\left\|\tau_{\rho}^{i}\right\|_{0, \Omega}^{2}+R_{12} \Delta t \sum_{e \in \Gamma_{h}} \frac{r_{\rho}^{2}}{|e|}\left\|\left[\tau_{\rho}^{i}\right]\right\|_{0, e}^{2}+R_{13} \Delta t\left\|\tau_{v}^{i}\right\|_{0, \Omega}^{2} \\
+R_{14} \Delta t\left(\frac{h^{2 \min \left(r_{\rho}+1, s_{\rho}\right)}}{r_{\rho}^{2 s_{\rho}}}+\frac{h^{2 \min \left(r_{v}+1, s_{v}\right)}}{r_{v}^{2 s_{v}}}\right) .
\end{gathered}
$$

For the term $T_{11}^{\rho}$ we obtain the following bound:

$$
\left|T_{11}^{\rho}\right| \leq \varepsilon_{11}^{\rho} \Delta t\left\|\tau_{\rho}^{i}\right\|_{0, \Omega}^{2}+R_{15} \Delta t \frac{h^{2 \min \left(r_{\rho}+1, s_{\rho}\right)}}{r_{\rho}^{2 s_{\rho}}}
$$

Next, we bound term $T_{12}^{\rho}$. First using a Taylor expansion with integral remainder we can write

$$
\begin{aligned}
& \widetilde{\rho}^{i+1}=\widetilde{\rho}^{i}+\Delta t \partial_{t} \widetilde{\rho}\left(t^{i}\right)+\frac{1}{2} \int_{t^{i}}^{t^{i+1}}\left(s-t^{i}\right) \partial_{t t} \widetilde{\rho}(s) \mathrm{ds}, \text { and we can bound } \\
& \frac{1}{2} \int_{t^{i}}^{t^{i+1}}\left(s-t^{i}\right) \partial_{t t} \widetilde{\rho}(s) \mathrm{d} s \leq \frac{1}{2}\left(\int_{t^{i}}^{t^{i+1}}\left(s-t^{i}\right)^{2}\right)^{\frac{1}{2}}\left(\int_{t^{i}}^{t^{i+1}}\left(\partial_{t t} \widetilde{\rho}(s)\right)^{2} \mathrm{ds}\right)^{\frac{1}{2}}
\end{aligned}
$$

Hence,

$$
4 \Delta t\left\|\widetilde{\rho}_{t}\left(t^{i}\right)-\frac{\widetilde{\rho}^{i+1}-\widetilde{\rho}^{i}}{\Delta t}\right\|_{0, \Omega}^{2} \leq \frac{1}{\Delta t} \int_{t^{i}}^{t^{i+1}}\left(s-t^{i}\right)^{2} \int_{t^{i}}^{t^{i+1}}\left\|\partial_{t t} \widetilde{\rho}(s)\right\|_{0, \Omega}^{2} \mathrm{ds},
$$

Hence, using the above estimates we obtain the following bound for $T_{12}^{\rho}$ :

$$
\left|T_{12}^{\rho}\right| \leq \varepsilon_{12}^{\rho} \Delta t\left\|\tau_{\rho}^{i}\right\|_{0, \Omega}^{2}+R_{16} \Delta t^{2} \int_{t^{i}}^{t^{i+1}}\left\|\partial_{t t} \widetilde{\rho}(s)\right\|_{0, \Omega}^{2} \mathrm{ds} .
$$

Next, we bound $T_{13}^{\rho}$ using Cauchy-Schwarz and the approximation inequality (2.2):

$$
\left|T_{13}^{\rho}\right| \leq 2 \Delta t \sum_{E \in \mathcal{E}_{h}}\left\|\nabla \tau_{\rho}^{i}\right\|_{0, E}\left\|\nabla \xi^{i}\right\|_{0, E} \leq \varepsilon_{13}^{\rho} \Delta t \mid\left\|\nabla \tau_{\rho}^{i}\right\| \|_{0, \Omega}^{2}+R_{17} \Delta t \frac{h^{2 \min \left(r_{\rho}+1, s_{\rho}\right)-2}}{r_{\rho}^{2 s_{\rho}-2}}
$$

Consider now term $T_{14}^{\rho}$ :

$$
\begin{gathered}
\left|T_{14}^{\rho}\right| \leq 2 \Delta t \sum_{e \in \Gamma_{h}}\left|\int_{e}\left\{\nabla \xi_{\rho}^{i} \cdot \mathbf{n}_{e}\right\}\left[\tau_{\rho}^{i}\right]\right| \\
\leq\left. 2 \Delta t \sum_{e \in \Gamma_{h}}\left\|\left\{\nabla \xi_{\rho}^{i} \cdot \mathbf{n}_{e}\right\}\right\|_{0, e}\left\|\left[\tau_{\rho}^{i}\right]\right\|\right|_{0, e}=\left.\left.2 \Delta t \sum_{e \in \Gamma_{h}} \frac{|e|^{\frac{1}{2}}}{r_{\rho}}\left\|\left\{\nabla \xi_{\rho}^{i} \cdot \mathbf{n}_{e}\right\}\right\|\right|_{0, e} \frac{r_{\rho}}{|e|^{\frac{1}{2}}}\left\|\left[\tau_{\rho}^{i}\right]\right\|\right|_{0, e}
\end{gathered}
$$


Next, using Cauchy-Schwarz and trace inequality (2.5) we obtain:

$$
\leq \varepsilon_{14}^{\rho} \Delta t \sum_{e \in \Gamma_{h}} \frac{r_{\rho}^{2}}{|e|}\left\|\left[\tau_{\rho}^{i}\right]\right\|_{0, e}^{2}+C \Delta t \frac{|e|}{r_{\rho}^{2}} \sum_{E \in \mathcal{E}_{h}}\left(h^{-1}\left\|\nabla \xi_{\rho}^{i}\right\|_{0, E}^{2}+h\left\|\nabla^{2} \xi_{\rho}^{i}\right\|_{0, E}^{2}\right)
$$

Finally, using the approximation result (2.2) we derive:

$$
\left|T_{14}^{\rho}\right| \leq \varepsilon_{14}^{\rho} \Delta t \sum_{e \in \Gamma_{h}} \frac{r_{\rho}^{2}}{|e|}\left\|\left[\tau_{\rho}^{i}\right]\right\|_{0, e}^{2}+R_{18} \Delta t \frac{h^{2 \min \left(r_{\rho}+1, s_{\rho}\right)-2}}{r_{\rho}^{2 s_{\rho}-2}}
$$

Next we bound term $T_{15}^{\rho}$ :

$$
\left|T_{15}^{\rho}\right| \leq \varepsilon 2 \Delta t \sum_{e \in \Gamma_{h}}\left|\int_{e}\left\{\nabla \tau_{\rho}^{i} \cdot \mathbf{n}_{e}\right\}\left[\xi_{\rho}^{i}\right]\right|
$$

Now using Lemma 2.3 and approximation result (2.3) we obtain:

$$
\leq \varepsilon 2 \Delta t \sum_{E \in \mathcal{E}_{h}}\left\|\nabla \tau_{\rho}^{i}\right\|_{0, E} \cdot C h^{-\frac{1}{2}} \cdot r_{\rho} \frac{h^{\min \left(r_{\rho}+1, s_{\rho}\right)-\frac{1}{2}}}{r_{\rho}^{s_{\rho}-\frac{1}{2}}}
$$

Next, applying Cauchy-Schwarz inequality we get the final estimate:

$$
\left|T_{15}^{\rho}\right| \leq \varepsilon_{15}^{\rho} \Delta t||\left|\nabla \tau_{\rho}^{i}\right| \|_{0, \Omega}^{2}+R_{19} \Delta t \frac{h^{2 \min \left(r_{\rho}+1, s_{\rho}\right)-2}}{r_{\rho}^{2 s_{\rho}-3}}
$$

Now, we bound term $T_{16}^{\rho}$ :

$$
\left|T_{16}^{\rho}\right| \leq 2 \Delta t \sigma_{\rho} \sum_{e \in \Gamma_{h}} \frac{r_{\rho}^{2}}{|e|}\left|\int_{e}\left[\xi_{\rho}^{i}\right]\left[\tau_{\rho}^{i}\right]\right| \leq\left.\left. 2 \Delta t \sigma_{\rho} \sum_{e \in \Gamma_{h}} \frac{r_{\rho}}{|e|^{\frac{1}{2}}}\left\|\left[\tau_{\rho}^{i}\right]\right\|\right|_{0, e}\left\|\left[\xi_{\rho}^{i}\right]\right\|\right|_{0, e} \frac{r_{\rho}}{|e|^{\frac{1}{2}}}
$$

Hence, we obtain the following bound for $T_{16}^{\rho}$ by using approximation result (2.3):

$$
\left|T_{16}^{\rho}\right| \leq \varepsilon_{16}^{\rho} \Delta t \sum_{e \in \Gamma_{h}} \frac{r_{\rho}^{2}}{|e|}\left\|\left[\tau_{\rho}^{i}\right]\right\|_{0, e}^{2}+R_{20} \Delta t \frac{h^{2 \min \left(r_{\rho}+1, s_{\rho}\right)-2}}{r_{\rho}^{2 s_{\rho}-3}}
$$

Terms $T_{17}^{\rho}$ and $T_{18}^{\rho}$ are bounded respectively using the approximation inequality (2.2) :

$$
\begin{gathered}
\left|T_{17}^{\rho}\right| \leq \varepsilon_{17}^{\rho} \Delta t|| \tau_{\rho}^{i} \|_{0, \Omega}^{2}+R_{21} \Delta t \frac{h^{2 \min \left(r_{\rho}+1, s_{\rho}\right)}}{r_{\rho}^{2 s_{\rho}}} \\
\left|T_{18}^{\rho}\right| \leq \varepsilon_{18}^{\rho} \Delta t\left|\left\|\nabla \tau_{\rho}^{i} \mid\right\|_{0, \Omega}^{2}+R_{22} \Delta t \frac{h^{2 \min \left(r_{\rho}+1, s_{\rho}\right)}}{r_{\rho}^{2 s_{\rho}}}\right.
\end{gathered}
$$

The last term that needs to be bound is the term $T_{1}^{\rho}$. To bound this term we again subtract (6.16) from (6.3) and choose $w^{\rho}=\tau_{\rho}^{i+1}-\tau_{\rho}^{i}$. Using similar techniques as for the estimation of 
(6.17), except now use inverse inequality to estimate $\nabla\left(\tau_{\rho}^{i+1}-\tau_{\rho}^{i}\right)$ and inequality (2.6) to estimate $\left\|\left[\tau_{\rho}^{i+1}-\tau_{\rho}^{i}\right]\right\|_{0, e}$, we obtain the following estimate:

$$
\begin{gathered}
\left\|\tau_{\rho}^{i+1}-\tau_{\rho}^{i}\right\|_{0, \Omega}^{2} \leq C_{1}^{k} \frac{\Delta t^{2} r_{\rho}^{4}}{h^{2}}\left\|\nabla \tau_{\rho}^{i}\right\|\left\|_{0, \Omega}^{2}+C_{2}^{k} \frac{\Delta t^{2} r_{\rho}^{4}}{h^{2}} \sum_{e \in \Gamma_{h}} \frac{r_{\rho}^{2}}{|e|}\right\|\left[\tau_{\rho}^{i}\right] \|_{0, e}^{2} \\
+C_{3}^{k} \frac{\Delta t^{2} r_{\rho}^{4}}{h^{2}}\left\|\tau_{\rho}^{i}\right\|_{0, \Omega}^{2}+C_{4}^{k} \frac{\Delta t^{2} r_{\rho}^{4}}{h^{2}}\left\|\tau_{u}^{i}\right\|_{0, \Omega}^{2}+C_{5}^{k} \frac{\Delta t^{2} r_{\rho}^{4}}{h^{2}}\left\|\tau_{v}^{i}\right\|_{0, \Omega}^{2} \\
+C_{6}^{k} \frac{\Delta t^{2} r_{\rho}^{4}}{h^{2}}\left(\frac{h^{2 \min \left(r_{\rho}+1, s_{\rho}\right)-2}}{r_{\rho}^{2 s_{\rho}-3}}+\frac{h^{2 \min \left(r_{u}+1, s_{u}\right)}}{r_{u}^{2 s_{u}}}+\frac{h^{2 \min \left(r_{v}+1, s_{v}\right)}}{r_{v}^{2 s_{v}}}\right)+C_{7}^{k} \Delta t^{3} \int_{t^{i}}^{t^{i+1}}\left\|\partial_{t t} \widetilde{\rho}(s)\right\|_{0, \Omega}^{2} \mathrm{ds}
\end{gathered}
$$

Some details of the derivation of the estimate (6.37) can be found in Appendix 9. Next, we combine estimates (6.19)-(6.36) together with (6.37), plug it in (6.17), and using again the assumptions $h<1$ and $r>1$ to obtain:

$$
\begin{gathered}
\left\|\tau_{\rho}^{i+1}\right\|_{0, \Omega}^{2}-\left\|\tau_{\rho}^{i}\right\|_{0, \Omega}^{2}+\Delta t\left(2-C_{1} \frac{\Delta t r_{\rho}^{4}}{h^{2}}\right)\left\|\nabla \tau_{\rho}^{i}\right\|\left\|_{0, \Omega}^{2}+\Delta t\left(2 \sigma_{\rho}-C_{2} \frac{\Delta t r_{\rho}^{4}}{h^{2}}\right) \sum_{e \in \Gamma_{h}} \frac{r_{\rho}^{2}}{|e|}\right\|\left[\tau_{\rho}^{i}\right] \|_{0, e}^{2} \\
\leq C_{3} \frac{\Delta t^{2} r_{\rho}^{4}}{h^{2}}\left\|\tau_{\rho}^{i}\right\|_{0, \Omega}^{2}+C_{4} \frac{\Delta t^{2} r_{\rho}^{4}}{h^{2}}\left\|\tau_{u}^{i}\right\|_{0, \Omega}^{2}+C_{5} \frac{\Delta t^{2} r_{\rho}^{4}}{h^{2}}\left\|\tau_{v}^{i}\right\|_{0, \Omega}^{2} \\
+C_{6} \frac{\Delta t^{2} r_{\rho}^{4}}{h^{2}}\left(\frac{h^{2 \min \left(r_{\rho}+1, s_{\rho}\right)-2}}{r_{\rho}^{2 s_{\rho}-3}}+\frac{h^{2 \min \left(r_{u}+1, s_{u}\right)}}{r_{u}^{2 s_{u}}}+\frac{h^{2 \min \left(r_{v}+1, s_{v}\right)}}{r_{v}^{2 s_{v}}}\right)+C_{7} \Delta t^{2} \int_{t_{i}}^{t_{i+1}}\left\|\partial_{t t} \widetilde{\rho}(s)\right\|_{0, \Omega}^{2} \mathrm{ds}
\end{gathered}
$$

Now sum (6.38) for $i=0, \ldots, n$ :

$$
\begin{aligned}
& \left\|\tau_{\rho}^{n+1}\right\|_{0, \Omega}^{2}-\left\|\tau_{\rho}^{0}\right\|_{0, \Omega}^{2}+\left(2-C_{1} \frac{\Delta t r_{\rho}^{4}}{h^{2}}\right) \sum_{i=0}^{n} \Delta t\left|\left\|\nabla \tau_{\rho}^{i} \mid\right\|\left\|_{0, \Omega}^{2}+\left(2 \sigma_{\rho}-C_{2} \frac{\Delta t r_{\rho}^{4}}{h^{2}}\right) \sum_{i=0}^{n} \Delta t \sum_{e \in \Gamma_{h}} \frac{r_{\rho}^{2}}{|e|}\right\|\left[\tau_{\rho}^{i}\right] \|_{0, e}^{2}\right. \\
& \leq C_{3} \frac{\Delta t r_{\rho}^{4}}{h^{2}} \sum_{i=0}^{n} \Delta t\left\|\tau_{\rho}^{i}\right\|_{0, \Omega}^{2}+C_{4} \frac{\Delta t r_{\rho}^{4}}{h^{2}} \sum_{i=0}^{n} \Delta t\left\|\tau_{u}^{i}\right\|_{0, \Omega}^{2}+C_{5} \frac{\Delta t r_{\rho}^{4}}{h^{2}} \sum_{i=0}^{n} \Delta t\left\|\tau_{v}^{i}\right\|_{0, \Omega}^{2} \\
& +C_{6} \frac{\Delta t r_{\rho}^{4}}{h^{2}} \sum_{i=0}^{n} \Delta t\left(\frac{h^{2 \min \left(r_{\rho}+1, s_{\rho}\right)-2}}{r_{\rho}^{2 s_{\rho}-3}}+\frac{h^{2 \min \left(r_{u}+1, s_{u}\right)}}{r_{u}^{2 s_{u}}}+\frac{h^{2 \min \left(r_{v}+1, s_{v}\right)}}{r_{v}^{2 s_{v}}}\right)+C_{7} \Delta t^{2} \sum_{i=0}^{n} \int_{t_{i}}^{t_{i+1}}\left\|\partial_{t t} \widetilde{\rho}(s)\right\|_{0, \Omega}^{2} \mathrm{ds}
\end{aligned}
$$

Now using induction hypothesis $S$ for $\sum_{i=0}^{n} \Delta t\left\|\tau_{u}^{i}\right\|_{0, \Omega}^{2}$ and $\sum_{i=0}^{n} \Delta t\left\|\tau_{v}^{i}\right\|_{0, \Omega}^{2}$, (6.10)-(6.11), we obtain:

$$
\begin{gathered}
\left\|\tau_{\rho}^{n+1}\right\|_{0, \Omega}^{2}+\left(2-C_{1} \frac{\Delta t r_{\rho}^{4}}{h^{2}}\right) \sum_{i=0}^{n} \Delta t\left|\left\|\nabla \tau_{\rho}^{i}\right\|\right|_{0, \Omega}^{2}+\left(2 \sigma_{\rho}-C_{2} \frac{\Delta t r_{\rho}^{4}}{h^{2}}\right) \sum_{i=0}^{n} \Delta t \sum_{e \in \Gamma_{h}} \frac{r_{\rho}^{2}}{|e|}\left\|\left[\tau_{\rho}^{i}\right]\right\|_{0, e}^{2} \\
\leq C_{3} \frac{\Delta t r_{\rho}^{4}}{h^{2}} \sum_{i=0}^{n} \Delta t\left\|\tau_{\rho}^{i}\right\|_{0, \Omega}^{2}+\left\|\tau_{\rho}^{0}\right\|_{0, \Omega}^{2}
\end{gathered}
$$




$$
\begin{aligned}
+\left(C_{4} \cdot C_{u}+C_{5} \cdot C_{v}\right. & \left.+C_{6}^{\star}\right) \frac{\Delta t r_{\rho}^{4}}{h^{2}}\left(\frac{h^{2 \min \left(r_{\rho}+1, s_{\rho}\right)-2}}{r_{\rho}^{2 s_{\rho}-3}}+\frac{h^{2 \min \left(r_{c}+1, s_{c}\right)-2}}{r_{c}^{2 s_{c}-3}}+\frac{h^{2 \min \left(r_{u}+1, s_{u}\right)-2}}{r_{u}^{2 s_{u}-3}}+\frac{h^{2 \min \left(r_{v}+1, s_{v}\right)-2}}{r_{v}^{2 s_{v}-3}}\right) \\
& +\left(C_{4} \cdot C_{u}^{t}+C_{5} \cdot C_{v}^{t}\right) \frac{\Delta t r_{\rho}^{4}}{h^{2}} \Delta t^{2}+C_{7} \Delta t^{2} \sum_{i=0}^{n} \int_{t_{i}}^{t_{i+1}}\left\|\partial_{t t} \widetilde{\rho}(s)\right\|_{0, \Omega}^{2} \mathrm{ds}
\end{aligned}
$$

Let us now define $C_{\text {max }}:=\max \left(C_{3}, \quad C_{4} \cdot C_{u}+C_{5} \cdot C_{v}+C_{6}^{\star}, \quad C_{4} \cdot C_{u}^{t}+C_{5} \cdot C_{v}^{t}\right)$, where constant $C_{\text {max }}$ depends on the properties of the exact solutions $\rho, u, v, T$ and domain $\Omega$ (it follows from the standard DG techniques and induction hypothesis for $i=n)$. Hence, we obtain:

$$
\begin{gathered}
\left\|\tau_{\rho}^{n+1}\right\|_{0, \Omega}^{2}+\left(2-C_{1} \frac{\Delta t r_{\rho}^{4}}{h^{2}}\right) \sum_{i=0}^{n} \Delta t\left|\left\|\nabla \tau_{\rho}^{i}\right\|\right|_{0, \Omega}^{2}+\left(2 \sigma_{\rho}-C_{2} \frac{\Delta t r_{\rho}^{4}}{h^{2}}\right) \sum_{i=0}^{n} \Delta t \sum_{e \in \Gamma_{h}} \frac{r_{\rho}^{2}}{|e|}\left\|\left[\tau_{\rho}^{i}\right]\right\|_{0, e}^{2} \\
\leq C_{\max } \frac{\Delta t r_{\rho}^{4}}{h^{2}} \sum_{i=0}^{n} \Delta t\left\|\tau_{\rho}^{i}\right\|_{0, \Omega}^{2}+\left\|\tau_{\rho}^{0}\right\|_{0, \Omega}^{2} \\
+C_{\max } \frac{\Delta t r_{\rho}^{4}}{h^{2}}\left(\frac{h^{2 \min \left(r_{\rho}+1, s_{\rho}\right)-2}}{r_{\rho}^{2 s_{\rho}-3}}+\frac{h^{2 \min \left(r_{c}+1, s_{c}\right)-2}}{r_{c}^{2 s_{c}-3}}+\frac{h^{2 \min \left(r_{u}+1, s_{u}\right)-2}}{r_{u}^{2 s_{u}-3}}+\frac{h^{2 \min \left(r_{v}+1, s_{v}\right)-2}}{r_{v}^{2 s_{v}-3}}\right) \\
+C_{\max } \frac{\Delta t r_{\rho}^{4}}{h^{2}} \Delta t^{2}+C_{7} \Delta t^{2} \sum_{i=0}^{n} \int_{t_{i}}^{t_{i+1}}\left\|\partial_{t t} \widetilde{\rho}(s)\right\|_{0, \Omega}^{2} \mathrm{ds}
\end{gathered}
$$

Next choosing $\Delta t \leq \min \left(\frac{h^{2}}{C_{\max r_{\rho}^{4}}}, \frac{h^{2}}{C_{1} r_{\rho}^{4}}, \frac{h^{2}}{C_{2} r_{\rho}^{4}}\right)$, setting penalty parameter $\sigma_{\rho}$ to be large enough, defining $C_{\text {min }}:=\min \left(1, \quad 2-C_{1} \frac{\Delta t r_{\rho}^{4}}{h^{2}}, \quad 2 \sigma_{\rho}-C_{2} \frac{\Delta t r_{\rho}^{4}}{h^{2}}\right)=1$, which can be done by choosing penalty parameter $\sigma_{\rho}$ large enough, and setting $C_{m m}:=\max \left(\frac{1}{C_{\min }}, \quad \frac{1+C_{7} \int_{0}^{T}\left\|\partial_{t t} \widetilde{\rho}(s)\right\|_{0, \Omega}^{2} \mathrm{ds}}{C_{m i n}}\right)=$ $1+C_{7} \int_{0}^{T}\left\|\partial_{t t} \widetilde{\rho}(s)\right\|_{0, \Omega}^{2}$ ds. Note that, now, the constant $C_{m m}$ is independent of the induction hypothesis. Hence, we derive:

$$
\begin{gathered}
\left\|\tau_{\rho}^{n+1}\right\|_{0, \Omega}^{2}+\sum_{i=0}^{n} \Delta t\left(\|\| \nabla \tau_{\rho}^{i} \mid\left\|_{0, \Omega}^{2}+\sum_{e \in \Gamma_{h}} \frac{r_{\rho}^{2}}{|e|}\right\|\left[\tau_{\rho}^{i}\right] \|_{0, e}^{2}\right) \\
\leq C_{m m} \sum_{i=0}^{n} \Delta t\left\|\tau_{\rho}^{i}\right\|_{0, \Omega}^{2}+C_{m m}\left(\frac{h^{2 \min \left(r_{\rho}+1, s_{\rho}\right)-2}}{r_{\rho}^{2 s_{\rho}-3}}+\frac{h^{2 \min \left(r_{c}+1, s_{c}\right)-2}}{r_{c}^{2 s_{c}-3}}+\frac{h^{2 \min \left(r_{u}+1, s_{u}\right)-2}}{r_{u}^{2 s_{u}-3}}+\frac{h^{2 \min \left(r_{v}+1, s_{v}\right)-2}}{r_{v}^{2 s_{v}-3}}\right) \\
+C_{m m}\left\|\tau_{\rho}^{0}\right\|_{0, \Omega}^{2}+C_{m m} \Delta t^{2},
\end{gathered}
$$

where $C_{m m}$ depends only on the properties of the exact solutions $\rho, T$ and domain $\Omega$. Now, applying discrete Gronwall's lemma to (6.42), we obtain the final estimate for $\tau_{\rho}^{n+1}$ :

$$
\begin{gathered}
\left\|\tau_{\rho}^{n+1}\right\|_{0, \Omega}^{2}+\sum_{i=0}^{n} \Delta t\left(\|\| \nabla \tau^{i}\|\|_{0, \Omega}^{2}+\sum_{e \in \Gamma_{h}} \frac{r_{\rho}^{2}}{|e|}\left\|\left[\tau^{i}\right]\right\|_{0, e}^{2}\right) \leq C^{\rho}\left(\frac{h^{2 \min \left(r_{\rho}+1, s_{\rho}\right)-2}}{r_{\rho}^{2 s_{\rho}-3}}\right. \\
\left.+\frac{h^{2 \min \left(r_{c}+1, s_{c}\right)-2}}{r_{c}^{2 s_{c}-3}}+\frac{h^{2 \min \left(r_{u}+1, s_{u}\right)-2}}{r_{u}^{2 s_{u}-3}}+\frac{h^{2 \min \left(r_{v}+1, s_{v}\right)-2}}{r_{v}^{2 s_{v}-3}}\right)+C_{\rho}^{t} \Delta t^{2}
\end{gathered}
$$


where constants $C^{\rho}, C_{\rho}^{t}$ depend only on the properties of the exact solutions $\rho$, domain $\Omega, T$ and independent of $n$. We now proceed in a similar way to the derivation of (6.43) for $\tau_{c}^{i+1}=\tau_{c}^{n+1}$ and we obtain:

$$
\begin{gathered}
\left\|\tau_{c}^{n+1}\right\|_{0, \Omega}^{2}+\sum_{i=0}^{n} \Delta t\left(\|\| \nabla \tau_{c}^{i} \mid\left\|_{0, \Omega}^{2}+\sum_{\Gamma_{h}} \frac{r_{c}^{2}}{|e|}\right\|\left[\tau_{c}^{i}\right] \|_{0, e}^{2}\right) \leq C^{c}\left(\frac{h^{2 \min \left(r_{\rho}+1, s_{\rho}\right)-2}}{r_{\rho}^{2 s_{\rho}-3}}+\frac{h^{2 \min \left(r_{c}+1, s_{c}\right)-2}}{r_{c}^{2 s_{c}-3}}\right. \\
\left.+\frac{h^{2 \min \left(r_{u}+1, s_{u}\right)-2}}{r_{u}^{2 s_{u}-3}}+\frac{h^{2 \min \left(r_{v}+1, s_{v}\right)-2}}{r_{v}^{2 s_{v}-3}}\right)+C_{c}^{t} \Delta t^{2}
\end{gathered}
$$

For the some details of the derivation of the above estimate (6.44) see Appendix 9.

Next, we proceed by proving that the induction hypothesis $S$ for $u_{\mathrm{DG}}^{n+1}$. Once again, by the consistency Lemma (4.1), the exact solution satisfies the following equation (compare it with $(3.5))$ :

$$
\begin{aligned}
& \int_{\Omega} \widetilde{u}\left(t^{i+1}\right) w^{u}+\sum_{E \in \mathcal{E}_{h}} \int_{E} \widetilde{c}^{i+1}\left(w^{u}\right)_{x}+\sum_{e \in \Gamma_{h}^{\mathrm{ver}}} \int_{e}\left(-c^{i+1}\right)_{u}^{* *} n_{x}\left[w^{u}\right]-\sum_{e \in \partial \Omega_{\mathrm{ver}}} \int_{e} \widetilde{c}^{i+1} n_{x} w^{u} \\
& +\sigma_{u} \sum_{e \in \Gamma_{h} \cup \partial \Omega_{\mathrm{ver}}} \frac{r_{u}^{2}}{|e|} \int_{e}\left[\widetilde{u}^{i+1}\right]\left[w^{u}\right]=-\int_{\Omega} \xi_{u}^{i+1} w^{u}-\sum_{E \in \mathcal{E}_{h}} \int_{E} \xi_{c}^{i+1}\left(w^{u}\right)_{x} \\
& +\sum_{e \in \partial \Omega_{\mathrm{ver}}} \int_{e} \xi_{c}^{i+1} n_{x} w^{u}-\sigma_{u} \sum_{e \in \Gamma_{h} \cup \partial \Omega_{\mathrm{ver}}} \frac{r_{u}^{2}}{|e|} \int_{e}\left[\xi_{u}^{i+1}\right]\left[w^{u}\right],
\end{aligned}
$$

where

$$
\left(-c^{i+1}\right)_{u}^{* *}:=-\frac{\left.a_{i}^{\text {out }} c^{i+1}\right|_{e} ^{E^{1}}-\left.a_{i}^{\text {in }} c^{i+1}\right|_{e} ^{E^{2}}}{a_{i}^{\text {out }}-a_{i}^{\text {in }}}-\frac{a_{i}^{\text {out }} a_{i}^{\text {in }}}{a_{i}^{\text {out }}-a_{i}^{\text {in }}}\left[u^{i+1}\right] .
$$

Subtracting equation (6.45) from (6.5) and choosing $w^{u}=\tau_{u}^{i+1}$, we obtain

$$
\begin{aligned}
& \left\|\tau_{u}^{i+1}\right\|_{0, \Omega}^{2}+\sigma_{u} \sum_{e \in \Gamma_{h} \cup \partial \Omega_{\mathrm{ver}}} \frac{r_{u}^{2}}{|e|}\left\|\left[\tau_{u}^{i+1}\right]\right\|_{0, \Omega}^{2} \\
& =-\sum_{E \in \mathcal{E}_{h}} \int_{E} \tau_{c}^{i+1}\left(\tau_{u}^{i+1}\right)_{x}-\sum_{e \in \Gamma_{h}^{\text {ver }}} \int_{e}\left(\left(-c_{\mathrm{DG}}^{i+1}\right)_{u}^{*}-\left(-c^{i+1}\right)_{u}^{* *}\right) n_{x}\left[\tau_{u}^{i+1}\right]+\sum_{e \in \partial \Omega_{\mathrm{ver}}} \int_{e} \tau_{c}^{i+1} n_{x}\left[\tau_{u}^{i+1}\right]+\int_{\Omega} \xi_{u}^{i+1} \tau_{u}^{i+1} \\
& \quad+\sum_{E \in \mathcal{E}_{h}} \int_{E} \xi_{c}^{i+1}\left(\tau_{u}^{i+1}\right)_{x}-\sum_{e \in \partial \Omega_{\mathrm{ver}}} \int_{e} \xi_{c}^{i+1} n_{x}\left[\tau_{u}^{i+1}\right]+\sigma_{u} \sum_{e \in \Gamma_{h} \cup \partial \Omega_{\mathrm{ver}}} \frac{r_{u}^{2}}{|e|} \int_{e}\left[\xi_{u}^{i+1}\right]\left[\tau_{u}^{i+1}\right]=: T_{1}^{u}+\ldots+T_{7}^{u},
\end{aligned}
$$

and bound each term on the RHS of (6.46).

Let us now bound term by term on the right-hand side of (6.46). Consider the term $T_{1}^{u}$. Using integration by parts, we get:

$$
-\int_{E} \tau_{c}^{i+1}\left(\tau_{u}^{i+1}\right)_{x}=-\left(-\int_{E} \tau_{u}^{i+1}\left(\tau_{c}^{i+1}\right)_{x}+\sum_{e \in \partial E} \int_{e} \tau_{c}^{i+1} \tau_{u}^{i+1} n_{x}\right),
$$

where $n_{x}$ denotes the $x$ - component of the outward normal vector to element $E$. 
Summing over all the elements $E$ we have:

$$
\begin{gathered}
-\left(-\sum_{E \in \mathcal{E}_{h}} \int_{E}\left(\tau_{c}^{i+1}\right)_{x} \tau_{u}^{i+1}+\sum_{E \in \mathcal{E}_{h}} \sum_{e \in \partial E} \int_{e} \tau_{c}^{i+1} \tau_{u}^{i+1} n_{x}\right) \\
=\sum_{E \in \mathcal{E}_{h}} \int_{E}\left(\tau_{c}^{i+1}\right)_{x} \tau_{u}^{i+1}-\sum_{e \in \Gamma_{h}^{\mathrm{ver}} \cup \partial \Omega} \int_{e}\left[\tau_{c}^{i+1} \tau_{u}^{i+1}\right] n_{x}
\end{gathered}
$$

Recalling the formula for the jump and average (2.1) we get:

$$
=\sum_{E \in \mathcal{E}_{h}} \int_{E}\left(\tau_{c}^{i+1}\right)_{x} \tau_{u}^{i+1}-\sum_{e \in \Gamma_{h}^{\mathrm{ver}}} \int_{e}\left[\tau_{c}^{i+1}\right]\left\{\tau_{u}^{i+1}\right\} n_{x}-\sum_{e \in \Gamma_{h}^{\mathrm{yer}}} \int_{e}\left[\tau_{u}^{i+1}\right]\left\{\tau_{c}^{i+1}\right\} n_{x}-\sum_{e \in \partial \Omega_{\mathrm{ver}}} \int_{e} \tau_{c}^{i+1}\left[\tau_{u}^{i+1}\right] n_{x}
$$

Hence, using the inequality (2.6), Cauchy-Schwarz's and Young's inequalities, using lemma 2.4 for $\left\|\tau_{c}^{i+1}\right\|$ and applying assumption (3.2), we have the following bound for $T_{1}^{u}$

$$
\left|T_{1}^{u}\right| \leq \varepsilon_{1}^{u}\left\|\tau_{u}^{i+1}\right\|_{0, \Omega}^{2}+U_{1} \sum_{e \in \Gamma_{h} \cup \partial \Omega_{\mathrm{ver}}} \frac{r_{u}^{2}}{|e|}\left\|\left[\tau_{u}^{i+1}\right]\right\|_{0, e}^{2}+U_{2}\left(\left\||| \nabla \tau_{c}^{i+1} \mid\right\|_{0, \Omega}^{2}+\sum_{e \in \Gamma_{h}} \frac{r_{c}^{2}}{|e|}\left\|\left[\tau_{c}^{i+1}\right]\right\|_{0, e}^{2}\right)
$$

A bound for $T_{2}^{u}$ can be obtained in a way similar to the bound on $T_{8}^{\rho}$ :

$$
\begin{aligned}
\left|T_{2}^{u}\right| & \leq \sum_{e \in \Gamma_{h}}\left(\left|\int_{e} \frac{a_{i}^{\text {out }}}{a_{i}^{\text {out }}-a_{i}^{\text {in }}}\left(\left(c_{\mathrm{DG}}^{i+1}\right)_{e}^{E^{1}}-\left(c^{i+1}\right)_{e}^{E^{1}}\right) n_{x}\left[\tau_{u}^{i+1}\right]\right|+\left|\int_{e} \frac{-a_{i}^{\text {in }}}{a_{i}^{\text {out }}-a_{i}^{\text {in }}}\left(\left(c_{\mathrm{DG}}^{i+1}\right)_{e}^{E^{2}}-\left(c^{i+1}\right)_{e}^{E^{2}}\right) n_{x}\left[\tau_{u}^{i+1}\right]\right|\right. \\
& \left.+\left|\int_{e} \frac{-a_{i}^{\text {in }} a_{i}^{\text {out }}}{a_{i}^{\text {out }}-a_{i}^{\text {in }}}\left[u_{\mathrm{DG}}^{i+1}-u^{i+1}\right] n_{x}\left[\tau_{u}^{i+1}\right]\right|\right):=\mathrm{I}+\mathrm{II}+\mathrm{III} .
\end{aligned}
$$

From (6.8) and (6.14), the first term on the RHS of (6.48) can be estimated by

$$
\mathrm{I} \leq \sum_{e \in \Gamma_{h}}\left(\left|\int_{e}\left(\tau_{c}^{i+1}\right)_{e}^{E^{1}} n_{x}\left[\tau_{u}^{i+1}\right]\right|+\left|\int_{e}\left(\xi_{c}^{i+1}\right)_{e}^{E^{1}} n_{x}\left[\tau_{u}^{i+1}\right]\right|\right):=\widetilde{\mathrm{I}}
$$

Using then the Cauchy-Schwarz inequality, the trace inequality (2.4), the inequality (2.6), and the assumption (3.2), we estimate $\widetilde{I}$ as follows:

$$
\widetilde{\mathrm{I}} \leq K K_{1}\left\|\tau_{c}^{i+1}\right\|_{0, \Omega}^{2}+K K_{2} \sum_{e \in \Gamma_{h}} \frac{r_{u}^{2}}{|e|}\left\|\left[\tau_{u}^{i+1}\right]\right\|_{0, e}^{2}+K K_{3} \frac{h^{2 \min \left(r_{c}+1, s_{c}\right)}}{r_{c}^{2 s_{c}}} .
$$

A similar bound can be derived for the second term on the RHS of (6.48). The third term on the RHS of (6.48) is similar to the third term on the RHS of (6.23), hence it can be bounded by

$$
\mathrm{III} \leq\left(\frac{K K_{4} \cdot h}{r_{u}^{2}}+\frac{1}{2}\right) \sum_{e \in \Gamma_{h}} \frac{r_{u}^{2}}{|e|}\left\|\left[\tau_{u}^{i+1}\right]\right\|_{0, e}^{2}+K K_{6} \cdot h \frac{h^{2 \min \left(r_{u}+1, s_{u}\right)-1}}{r_{u}^{2 s_{u}}} .
$$

By choosing $h$ small enough, we obtain:

$$
\mathrm{III} \leq \sum_{e \in \Gamma_{h}} \frac{r_{u}^{2}}{|e|}\left\|\left[\tau_{u}^{i+1}\right]\right\|_{0, e}^{2}+\frac{h^{2 \min \left(r_{u}+1, s_{u}\right)-1}}{r_{u}^{2 s_{u}}} .
$$


Combining the above bounds on I, II, and III, and using lemma 2.4 we arrive at

$$
\begin{gathered}
\left|T_{2}^{u}\right| \leq U_{3}\left(\left\||| \nabla \tau_{c}^{i+1} \mid\right\|_{0, \Omega}^{2}+\sum_{e \in \Gamma_{h}} \frac{r_{c}^{2}}{|e|}\left\|\left[\tau_{c}^{i+1}\right]\right\|_{0, e}^{2}\right)+U_{4} \sum_{e \in \Gamma_{h} \cup \partial \Omega_{\mathrm{ver}}} \frac{r_{u}^{2}}{|e|}\left\|\left[\tau_{u}^{i+1}\right]\right\|_{0, e}^{2} \\
+U_{5}\left(\frac{h^{2 \min \left(r_{u}+1, s_{u}\right)-1}}{r_{u}^{2 s_{u}}}+\frac{h^{2 \min \left(r_{c}+1, s_{c}\right)}}{r_{c}^{2 s_{c}}}\right) .
\end{gathered}
$$

To bound the term $T_{3}^{u}$, we use the Cauchy-Schwarz inequality, Young's inequality, the inequality (2.6), and lemma 2.4 which yield

$$
\left|T_{3}^{u}\right| \leq U_{6}\left(||\left|\nabla \tau_{c}^{i+1}\right|\left\|_{0, \Omega}^{2}+\sum_{e \in \Gamma_{h}} \frac{r_{c}^{2}}{|e|}\right\|\left[\tau_{c}^{i+1}\right] \|_{0, e}^{2}\right)+U_{7} \sum_{e \in \partial \Omega_{\mathrm{ver}}} \frac{r_{u}^{2}}{|e|}\left\|\left[\tau_{u}^{i+1}\right]\right\|_{0, e}^{2} .
$$

The term $T_{4}^{u}$ is bounded with the help of Cauchy-Schwarz inequality, Young's inequality, and the approximation inequality (2.2):

$$
\left|T_{4}^{u}\right| \leq \varepsilon_{2}^{u}\left\|\tau_{u}^{i+1}\right\|_{0, \Omega}^{2}+U_{8} \frac{h^{2 \min \left(r_{u}+1, s_{u}\right)}}{r_{u}^{2 s_{u}}}
$$

Using similar idea as for the term $T_{1}^{u}$, we can rewrite $T_{5}^{u}$ in the form

$$
\begin{aligned}
& T_{5}^{u}=-\sum_{E \in \mathcal{E}_{h}} \int_{E}\left(\xi_{c}^{i+1}\right)_{x} \tau_{u}^{i+1}+\sum_{e \in \Gamma_{h}^{\mathrm{ver}}} \int_{e}\left[\xi_{c}^{i+1}\right]\left\{\tau_{u}^{i+1}\right\} n_{x}+\sum_{e \in \Gamma_{h}^{\mathrm{ver}}} \int_{e}\left[\tau_{u}^{i+1}\right]\left\{\xi_{c}^{i+1}\right\} n_{x} \\
& +\sum_{e \in \partial \Omega_{\mathrm{ver}}} \int_{e} \xi_{c}^{i+1}\left[\tau_{u}^{i+1}\right] n_{x}:=\widetilde{T}_{5}^{u} .
\end{aligned}
$$

We then use Lemma 2.1 and Lemma 2.3 to obtain

$$
\left|\widetilde{T}_{5}\right| \leq \varepsilon_{3}^{u}\left\|\tau_{u}^{i+1}\right\|_{0, \Omega}^{2}+\widetilde{U_{9}} \sum_{e \in \Gamma_{h} \cup \partial \Omega_{\mathrm{ver}}} \frac{r_{u}^{2}}{|e|}\left\|\left[\tau_{u}^{i+1}\right]\right\|_{0, e}^{2}+U_{9} \frac{h^{2 \min \left(r_{c}+1, s_{c}\right)-2}}{r_{c}^{2 s_{c}-3}} .
$$

The term $T_{6}^{u}$ is bounded using the Cauchy-Schwarz inequality, the trace inequality (2.4), and the approximation inequality (2.2):

$$
\left|T_{6}^{u}\right| \leq U_{10} \sum_{e \in \partial \Omega_{\mathrm{ver}}} \frac{r_{u}^{2}}{|e|}\left\|\left[\tau_{u}^{i+1}\right]\right\|_{0, e}^{2}+U_{11} \frac{h^{2 \min \left(r_{c}+1, s_{c}\right)}}{r_{c}^{2 s_{c}}} .
$$

The last term $T_{7}^{u}$ is bounded using the approximation result (2.3). Hence,

$$
\left|T_{7}^{u}\right| \leq U_{12} \sum_{e \in \Gamma_{h} \cup \partial \Omega_{\mathrm{ver}}} \frac{r_{u}^{2}}{|e|}\left\|\left[\tau_{u}^{i+1}\right]\right\|_{0, e}^{2}+U_{13} \frac{h^{2 \min \left(r_{u}+1, s_{u}\right)-2}}{r_{u}^{2 s_{u}-3}} .
$$

After obtaining the estimates (6.47)-(6.55), we plug them into (6.46) and use the assumption $h<1, r>1$, assumption (3.2), choose the penalty parameters large enough and an appropriate scaling to obtain 


$$
\begin{gathered}
\left\|\tau_{u}^{i+1}\right\|_{0, \Omega}^{2}+\sum_{e \in \Gamma_{h} \cup \partial \Omega_{\mathrm{ver}}} \frac{r_{u}^{2}}{|e|}\left\|\left[\tau_{u}^{i+1}\right]\right\|_{0, e}^{2} \\
\leq C_{u}^{1}\left(\left\|\left|\nabla \tau_{c}^{i+1}\right|\right\|_{0, \Omega}^{2}+\sum_{e \in \Gamma_{h}} \frac{r_{c}^{2}}{|e|}\left\|\left[\tau_{c}^{i+1}\right]\right\|_{0, e}^{2}\right)+C_{u}^{2}\left(\frac{h^{2 \min \left(r_{c}+1, s_{c}\right)-2}}{r_{c}^{2 s_{c}-3}}+\frac{h^{2 \min \left(r_{u}+1, s_{u}\right)-2}}{r_{u}^{2 s_{u}-3}}\right) .
\end{gathered}
$$

Next multiply both sides of (6.56) by $\Delta t$ and sum for $i=-1, \ldots, n$ to get

$$
\begin{gathered}
\sum_{i=-1}^{n} \Delta t\left\|\tau_{u}^{i+1}\right\|_{0, \Omega}^{2}+\sum_{i=-1}^{n} \Delta t \sum_{e \in \Gamma_{h} \cup \partial \Omega_{\mathrm{ver}}} \frac{r_{u}^{2}}{|e|}\left\|\left[\tau_{u}^{i+1}\right]\right\|_{0, e}^{2} \\
\leq C_{u}^{3}\left(\Delta t\left|\left\|\nabla \tau_{c}^{n+1} \mid\right\|_{0, \Omega}^{2}+\Delta t \sum_{e \in \Gamma_{h}} \frac{r_{c}^{2}}{|e|}\left\|\left[\tau_{c}^{n+1}\right]\right\|_{0, e}^{2}\right)\right. \\
+\sum_{i=0}^{n} C_{u}^{4} \Delta t\left(\left\||| \nabla \tau_{c}^{i} \mid\right\|_{0, \Omega}^{2}+\sum_{e \in \Gamma_{h}} \frac{r_{c}^{2}}{|e|}\left\|\left[\tau_{c}^{i}\right]\right\|_{0, e}^{2}\right)+\sum_{i=-1}^{n} C_{u}^{5} \Delta t\left(\frac{h^{2 \min \left(r_{c}+1, s_{c}\right)-2}}{r_{c}^{2 s_{c}-3}}+\frac{h^{2 \min \left(r_{u}+1, s_{u}\right)-2}}{r_{u}^{2 s_{u}-3}}\right) .
\end{gathered}
$$

Next, using the inverse inequality, inequality (2.6) we obtain:

$$
\begin{gathered}
\sum_{i=-1}^{n} \Delta t\left\|\tau_{u}^{i+1}\right\|_{0, \Omega}^{2}+\sum_{i=-1}^{n} \Delta t \sum_{e \in \Gamma_{h} \cup \partial \Omega_{\mathrm{ver}}} \frac{r_{u}^{2}}{|e|}\left\|\left[\tau_{u}^{i+1}\right]\right\|_{0, e}^{2} \\
\leq C_{u}^{6}\left(\frac{\Delta t r_{c}^{4}}{h^{2}}\left\|\tau_{c}^{n+1}\right\|_{0, \Omega}^{2}+\frac{\Delta t r_{c}^{4}}{h^{2}}\left\|\tau_{c}^{n+1}\right\|_{0, \Omega}^{2}\right) \\
+\sum_{i=0}^{n} C_{u}^{7} \Delta t\left(\left\|\mid \nabla \tau_{c}^{i}\right\|\left\|_{0, \Omega}^{2}+\sum_{e \in \Gamma_{h}} \frac{r_{c}^{2}}{|e|}\right\|\left[\tau_{c}^{i}\right] \|_{0, e}^{2}\right)+\sum_{i=-1}^{n} C_{u}^{8} \Delta t\left(\frac{h^{2 \min \left(r_{c}+1, s_{c}\right)-2}}{r_{c}^{2 s_{c}-3}}+\frac{h^{2 \min \left(r_{u}+1, s_{u}\right)-2}}{r_{u}^{2 s_{u}-3}}\right) .
\end{gathered}
$$

Final estimate is obtained by choosing $\Delta t \leq C \frac{h^{2}}{r_{c}^{4}}$ and by using the estimate (6.44):

$$
\begin{gathered}
\sum_{i=-1}^{n} \Delta t\left\|\tau_{u}^{i+1}\right\|_{0, \Omega}^{2}+\sum_{i=-1}^{n} \Delta t \sum_{e \in \Gamma_{h} \cup \partial \Omega_{\mathrm{ver}}} \frac{r_{u}^{2}}{|e|}\left\|\left[\tau_{u}^{i+1}\right]\right\|_{0, e}^{2} \\
\leq C_{u}\left(\frac{h^{2 \min \left(r_{\rho}+1, s_{\rho}\right)-2}}{r_{\rho}^{2 s_{\rho}-3}}+\frac{h^{2 \min \left(r_{c}+1, s_{c}\right)-2}}{r_{c}^{2 s_{c}-3}}+\frac{h^{2 \min \left(r_{u}+1, s_{u}\right)-2}}{r_{u}^{2 s_{u}-3}}+\frac{h^{2 \min \left(r_{v}+1, s_{v}\right)-2}}{r_{v}^{2 s_{v}-3}}\right)+C_{u}^{t} \Delta t^{2} .
\end{gathered}
$$

The estimate (6.59) proves the induction hypothesis (6.10) for $i=n+1$ :

$$
\begin{gathered}
\sum_{i=0}^{n+1} \Delta t\left\|\tau_{u}^{i}\right\|_{0, \Omega}^{2} \leq C_{u}\left(\frac{h^{2 \min \left(r_{\rho}+1, s_{\rho}\right)-2}}{r_{\rho}^{2 s_{\rho}-3}}+\frac{h^{2 \min \left(r_{c}+1, s_{c}\right)-2}}{r_{c}^{2 s_{c}-3}}\right. \\
\left.+\frac{h^{2 \min \left(r_{u}+1, s_{u}\right)-2}}{r_{u}^{2 s_{u}-3}}+\frac{h^{2 \min \left(r_{v}+1, s_{v}\right)-2}}{r_{v}^{2 s_{v}-3}}\right)+C_{u}^{t} \Delta t^{2}
\end{gathered}
$$


Induction hypothesis $S,(6.12)$ can be shown using similar techniques as in (6.10), see details in Appendix 9. In the same way, we prove the induction hypothesis $S$ for $\tau_{v}$ and show that:

$$
\begin{gathered}
\sum_{i=0}^{n+1} \Delta t\left\|\tau_{v}^{i}\right\|_{0, \Omega}^{2}+\sum_{i=0}^{n+1} \Delta t \sum_{e \in \Gamma_{h} \cup \partial \Omega_{\mathrm{hor}}} \frac{r_{v}^{2}}{|e|}\left\|\left[\tau_{v}^{i}\right]\right\|_{0, e}^{2} \\
\leq C_{v}\left(\frac{h^{2 \min \left(r_{\rho}+1, s_{\rho}\right)-2}}{r_{\rho}^{2 s_{\rho}-3}}+\frac{h^{2 \min \left(r_{c}+1, s_{c}\right)-2}}{r_{c}^{2 s_{c}-3}}+\frac{h^{2 \min \left(r_{u}+1, s_{u}\right)-2}}{r_{u}^{2 s_{u}-3}}+\frac{h^{2 \min \left(r_{v}+1, s_{v}\right)-2}}{r_{v}^{2 s_{v}-3}}\right)+C_{v}^{t} \Delta t^{2} .
\end{gathered}
$$

\section{Runge-Kutta Second Order Time Discretization}

Find a discrete in time solution

$$
\left(\rho_{\mathrm{DG}}^{i+1}, c_{\mathrm{DG}}^{i+1}, u_{\mathrm{DG}}^{i+1}, v_{\mathrm{DG}}^{i+1}\right) \in \mathcal{W}_{r_{\rho}, h}^{\rho} \times \mathcal{W}_{r_{c}, h}^{c} \times \mathcal{W}_{r_{u}, h}^{u} \times \mathcal{W}_{r_{v}, h}^{v},
$$

which satisfies the following weak formulation for the chemotaxis system (1.2):

$$
\begin{aligned}
& \int_{\Omega} z_{\mathrm{DG}, \rho}^{i} w^{\rho}=\int_{\Omega} \rho_{\mathrm{DG}}^{i} w^{\rho}-\Delta t\left(\sum_{E \in \mathcal{E}_{h}} \int_{E} \nabla \rho_{\mathrm{DG}}^{i} \nabla w^{\rho}-\sum_{e \in \Gamma_{h}} \int_{e}\left\{\nabla \rho_{\mathrm{DG}}^{i} \cdot \mathbf{n}_{e}\right\}\left[w^{\rho}\right]+\varepsilon \sum_{e \in \Gamma_{h}} \int_{e}\left\{\nabla w^{\rho} \cdot \mathbf{n}_{e}\right\}\left[\rho_{\mathrm{DG}}^{i}\right]\right. \\
& +\sigma_{\rho} \sum_{e \in \Gamma_{h}} \frac{r_{\rho}^{2}}{|e|} \int_{e}\left[\rho_{\mathrm{DG}}^{i}\right]\left[w^{\rho}\right]-\sum_{E \in \mathcal{E}_{h}} \int_{E} \chi \rho_{\mathrm{DG}}^{i} u_{\mathrm{DG}}^{i}\left(w^{\rho}\right)_{x}+\sum_{e \in \Gamma_{h}^{\text {ver }}} \int_{e}\left(\chi \rho_{\mathrm{DG}}^{i} u_{\mathrm{DG}}^{i}\right)^{*} n_{x}\left[w^{\rho}\right] \\
& \left.-\sum_{E \in \mathcal{E}_{h}} \int_{E} \chi \rho_{\mathrm{DG}}^{i} v_{\mathrm{DG}}^{i}\left(w^{\rho}\right)_{y}+\sum_{e \in \Gamma_{h}^{\text {hor }}} \int_{e}\left(\chi \rho_{\mathrm{DG}}^{i} v_{\mathrm{DG}}^{i}\right)^{*} n_{y}\left[w^{\rho}\right]\right), \\
& \int_{\Omega} z_{\mathrm{DG}, \mathrm{c}}^{i} w^{c}=\int_{\Omega} c_{\mathrm{DG}}^{i} w^{c}-\Delta t\left(\sum_{E \in \mathcal{E}_{h}} \int_{E} \nabla c_{\mathrm{DG}}^{i} \nabla w^{c}-\sum_{e \in \Gamma_{h}} \int_{e}\left\{\nabla c_{\mathrm{DG}}^{i} \cdot \mathbf{n}_{e}\right\}\left[w^{c}\right]+\varepsilon \sum_{e \in \Gamma_{h}} \int_{e}\left\{\nabla w^{c} \cdot \mathbf{n}_{e}\right\}\left[c_{\mathrm{DG}}^{i}\right]\right. \\
& \left.+\sigma_{c} \sum_{e \in \Gamma_{h}} \frac{r_{c}^{2}}{|e|} \int_{e}\left[c_{\mathrm{DG}}^{i}\right]\left[w^{c}\right]+\int_{\Omega} c_{\mathrm{DG}}^{i} w^{c}-\int_{\Omega} \rho_{\mathrm{DG}}^{i} w^{c}\right), \\
& \int_{\Omega} z_{\mathrm{DG}, \mathrm{u}}^{i} w^{u}+\sigma_{u} \sum_{e \in \Gamma_{h} \cup \partial \Omega_{\mathrm{ver}}} \frac{r_{u}^{2}}{|e|} \int_{e}\left[z_{\mathrm{DG}, \mathrm{u}}^{i}\right]\left[w^{u}\right]=-\left(\sum_{E \in \mathcal{E}_{h}} \int_{E} z_{\mathrm{DG}, \mathrm{c}}^{i}\left(w^{u}\right)_{x}+\sum_{e \in \Gamma_{h}^{\mathrm{hor}}} \int_{e}\left(-z_{\mathrm{DG}, \mathrm{c}}^{i}\right)_{u}^{*} n_{x}\left[w^{u}\right]\right. \\
& \left.-\sum_{e \in \partial \Omega_{\mathrm{ver}}} \int_{e} z_{\mathrm{DG}, \mathrm{c}}^{i} n_{x} w^{u}\right) \\
& \int_{\Omega} z_{\mathrm{DG}, \mathrm{v}}^{i} w^{v}+\sigma_{v} \sum_{e \in \Gamma_{h} \cup \partial \Omega_{\mathrm{hor}}} \frac{r_{v}^{2}}{|e|} \int_{e}\left[z_{\mathrm{DG}, \mathrm{v}}^{i}\right]\left[w^{v}\right]=-\left(\sum_{E \in \mathcal{E}_{h}} \int_{E} z_{\mathrm{DG}, \mathrm{c}}^{i}\left(w^{v}\right)_{y}+\sum_{e \in \Gamma_{h}^{\mathrm{hor}}} \int_{e}\left(-z_{\mathrm{DG}, \mathrm{c}}^{i}\right)_{v}^{*} n_{y}\left[w^{v}\right]\right. \\
& \left.-\sum_{e \in \partial \Omega_{\mathrm{hor}}} \int_{e} z_{\mathrm{DG}, \mathrm{c}}^{i} n_{y} w^{v}\right) \text {, } \\
& \int_{\Omega} \rho_{\mathrm{DG}}^{i+1} w^{\rho}=\frac{1}{2} \int_{\Omega} \rho_{\mathrm{DG}}^{i} w^{\rho}+\frac{1}{2} \int_{\Omega} z_{\mathrm{DG}, \rho}^{i} w^{\rho}-0.5 \Delta t\left(\sum_{E \in \mathcal{E}_{h}} \int_{E} \nabla z_{\mathrm{DG}, \rho}^{i} \nabla w^{\rho}-\sum_{e \in \Gamma_{h}} \int_{e}\left\{\nabla z_{\mathrm{DG}, \rho}^{i} \cdot \mathbf{n}_{e}\right\}\left[w^{\rho}\right]\right.
\end{aligned}
$$




$$
\begin{aligned}
& +\varepsilon \sum_{e \in \Gamma_{h}} \int_{e}\left\{\nabla w^{\rho} \cdot \mathbf{n}_{e}\right\}\left[z_{\mathrm{DG}, \rho}^{i}\right]+\sigma_{\rho} \sum_{e \in \Gamma_{h}} \frac{r_{\rho}^{2}}{|e|} \int_{e}\left[z_{\mathrm{DG}, \rho}^{i}\right]\left[w^{\rho}\right]-\sum_{E \in \mathcal{E}_{h}} \int_{E} \chi z_{\mathrm{DG}, \rho}^{i} z_{\mathrm{DG}, u}^{i}\left(w^{\rho}\right)_{x} \\
& \left.+\sum_{e \in \Gamma_{h}^{\mathrm{ver}}} \int_{e}\left(\chi z_{\mathrm{DG}, \rho}^{i} z_{\mathrm{DG}, u}^{i}\right)^{*} n_{x}\left[w^{\rho}\right]-\sum_{E \in \mathcal{E}_{h}} \int_{E} \chi z_{\mathrm{DG}, \rho}^{i} z_{\mathrm{DG}, v}^{i}\left(w^{\rho}\right)_{y}+\sum_{e \in \Gamma_{h}^{\mathrm{hor}}} \int_{e}\left(\chi z_{\mathrm{DG}, \rho}^{i} z_{\mathrm{DG}, v}^{i}\right)^{*} n_{y}\left[w^{\rho}\right]\right), \\
& \int_{\Omega} c_{\mathrm{DG}}^{i+1} w^{c}=\frac{1}{2} \int_{\Omega} c_{\mathrm{DG}}^{i} w^{c}+\frac{1}{2} \int_{\Omega} z_{\mathrm{DG}, \mathrm{c}}^{i} w^{c}-0.5\left(\sum_{E \in \mathcal{E}_{h}} \int_{E} \nabla z_{\mathrm{DG}, \mathrm{c}}^{i} \nabla w^{c}-\sum_{e \in \Gamma_{h}} \int_{e}\left\{\nabla z_{\mathrm{DG}, \mathrm{c}}^{i} \cdot \mathbf{n}_{e}\right\}\left[w^{c}\right]\right. \\
& \left.+\varepsilon \sum_{e \in \Gamma_{h}} \int_{e}\left\{\nabla w^{c} \cdot \mathbf{n}_{e}\right\}\left[z_{\mathrm{DG}, \mathrm{c}}^{i}\right]+\sigma_{c} \sum_{e \in \Gamma_{h}} \frac{r_{c}^{2}}{|e|} \int_{e}\left[z_{\mathrm{DG}, \mathrm{c}}^{i}\right]\left[w^{c}\right]+\int_{\Omega} z_{\mathrm{DG}, \mathrm{c}}^{i} w^{c}-\int_{\Omega} z_{\mathrm{DG}, \rho}^{i} w^{c}\right), \\
& \int_{\Omega} u_{\mathrm{DG}}^{i+1} w^{u}+\sigma_{u} \sum_{e \in \Gamma_{h} \cup \partial \Omega_{\mathrm{ver}}} \frac{r_{u}^{2}}{|e|} \int_{e}\left[u_{\mathrm{DG}}^{i+1}\right]\left[w^{u}\right]=-\left(\sum_{\mathrm{DG}}^{i+1}\left(w^{u}\right)_{x}+\sum_{e \in \Gamma_{h}^{\mathrm{ver}}} \int_{e}\left(-c_{\mathrm{DG}}^{i+1}\right)_{u}^{*} n_{x}\left[w^{u}\right]\right. \\
& \left.-\sum_{e \in \partial \Omega_{\mathrm{ver}}} \int_{e} c_{\mathrm{DG}}^{i+1} n_{x} w^{u}\right), \\
& \int_{\Omega} v_{\mathrm{DG}}^{i+1} w^{v}+\sigma_{v} \sum_{e \in \Gamma_{h} \cup \partial \Omega_{\mathrm{hor}}} \frac{r_{v}^{2}}{|e|} \int_{e}\left[v_{\mathrm{DG}}^{i+1}\right]\left[w^{v}\right]=-\left(\sum_{E \in \mathcal{E}_{h}} \int_{E} c_{\mathrm{DG}}^{i+1}\left(w^{v}\right)_{y}+\sum_{e \in \Gamma_{h}^{\mathrm{hor}}} \int_{e}\left(-c_{\mathrm{DG}}^{i+1}\right)_{v}^{*} n_{y}\left[w^{v}\right]\right. \\
& \left.\quad \sum_{e \in \partial \Omega_{\mathrm{hor}}} \int_{e} c_{\mathrm{DG}}^{i+1} n_{y} w^{v}\right),
\end{aligned}
$$

Again, we use (3.8) to approximate the convective terms in the scheme above, with the one-sided local speeds given by:

$$
\begin{aligned}
& a_{i, u}^{\text {out }}:=\max \left(\left(\chi u_{\mathrm{DG}}^{i}\right)_{e}^{E^{1}},\left(\chi u_{\mathrm{DG}}^{i}\right)_{e}^{E^{2}}, 0\right), \quad a_{i, u}^{\text {in }}:=\min \left(\left(\chi u_{\mathrm{DG}}^{i}\right)_{e}^{E^{1}},\left(\chi u_{\mathrm{DG}}^{i}\right)_{e}^{E^{2}}, 0\right), \\
& b_{i, v}^{\text {out }}:=\max \left(\left(\chi v_{\mathrm{DG}}^{i}\right)_{e}^{E^{1}},\left(\chi v_{\mathrm{DG}}^{i}\right)_{e}^{E^{2}}, 0\right), \quad b_{i, v}^{\text {in }}:=\min \left(\left(\chi v_{\mathrm{DG}}^{i}\right)_{e}^{E^{1}},\left(\chi v_{\mathrm{DG}}^{i}\right)_{e}^{E^{2}}, 0\right), \\
& a_{i, z_{u}}^{\text {out }}:=\max \left(\left(\chi z_{\mathrm{DG}, \mathrm{u}}^{i}\right)_{e}^{E^{1}},\left(\chi z_{\mathrm{DG}, \mathrm{u}}^{i}\right)_{e}^{E^{2}}, 0\right), \quad a_{i, z_{u}}^{\text {in }}:=\min \left(\left(\chi z_{\mathrm{DG}, \mathrm{u}}^{i}\right)_{e}^{E^{1}},\left(\chi z_{\mathrm{DG}, \mathrm{u}}^{i}\right)_{e}^{E^{2}}, 0\right), \\
& b_{i, z_{v}}^{\text {out }}:=\max \left(\left(\chi z_{\mathrm{DG}, \mathrm{v}}^{i}\right)_{e}^{E^{1}},\left(\chi z_{\mathrm{DG}, \mathrm{v}}^{i}\right)_{e}^{E^{2}}, 0\right), \quad b_{i, z_{v}}^{\text {in }}:=\min \left(\left(\chi z_{\mathrm{DG}, \mathrm{v}}^{i}\right)_{e}^{E^{1}},\left(\chi z_{\mathrm{DG}, \mathrm{v}}^{i}\right)_{e}^{E^{2}}, 0\right),
\end{aligned}
$$

Denote by $m_{1}:=u, z_{u}, \quad m_{2}:=v, z_{v}$ and notice that we make the same settings here as in the Remark in section 3. Also notice that the inequalities similar to (3.10),

$$
\frac{a_{i, m_{1}}^{\text {out }}}{a_{i, m_{1}}^{\text {out }}-a_{i, m_{1}}^{\text {in }}} \leq 1, \quad \frac{-a_{i, m_{1}}^{\text {in }}}{a_{i, m_{1}}^{\text {out }}-a_{i, m_{1}}^{\text {in }}} \leq 1, \quad \frac{b_{i, m_{2}}^{\text {out }}}{b_{i, m_{2}}^{\text {out }}-b_{i, m_{2}}^{\text {in }}} \leq 1, \quad \text { and } \quad \frac{-b_{i, m_{2}}^{\text {in }}}{b_{i, m_{2}}^{\text {out }}-b_{i, m_{2}}^{\text {in }}} \leq 1,
$$

which are needed in our convergence proof, are satisfied for the local speeds defined in (7.9) as well (for simplicity, we assume that $a_{i, m_{1}}^{\text {out }}-a_{i, m_{1}}^{\text {in }} \neq 0$ and $b_{i, m_{2}}^{\text {out }}-b_{i, m_{2}}^{\text {in }} \neq 0$ throughout the computational domain) and the initial conditions:

$$
\begin{aligned}
& \int_{\Omega} \rho_{\mathrm{DG}}^{0} w^{\rho}=\int_{\Omega} \rho^{0} w^{\rho}, \quad \int_{\Omega} c_{\mathrm{DG}}^{0} w^{c}=\int_{\Omega} c^{0} w^{c}, \\
& \int_{\Omega} u_{\mathrm{DG}}^{0} w^{u}=\int_{\Omega} u^{0} w^{u}, \quad \int_{\Omega} v_{\mathrm{DG}}^{0} w^{v}=\int_{\Omega} v^{0} w^{v} .
\end{aligned}
$$


Now, let us use a similar idea to [41] and define new variables $z_{\rho}, z_{c}, z_{u}, z_{v}$ :

$$
\begin{aligned}
& z_{\rho}(x, y, t)=\rho(x, y, t)+\rho_{t}(x, y, t) \Delta t \\
& z_{c}(x, y, t)=c(x, y, t)+c_{t}(x, y, t) \Delta t \\
& z_{u}(x, y, t)=u(x, y, t)+u_{t}(x, y, t) \Delta t \\
& z_{v}(x, y, t)=v(x, y, t)+v_{t}(x, y, t) \Delta t
\end{aligned}
$$

Next, using the regularity of the density $\rho$, the concentration $c$, and Taylor expansion, we obtain for $\rho$ and $c$ :

$$
\begin{aligned}
& \rho(x, y, t+\Delta t)-\rho(x, y, t)-\rho_{t}(x, y, t) \frac{\Delta t}{2}-\rho_{t}(x, y, t+\Delta t) \frac{\Delta t}{2}=O\left(\Delta t^{3}\right) \\
& c(x, y, t+\Delta t)-c(x, y, t)-c_{t}(x, y, t) \frac{\Delta t}{2}-c_{t}(x, y, t+\Delta t) \frac{\Delta t}{2}=O\left(\Delta t^{3}\right)
\end{aligned}
$$

Hence, we obtain:

$$
\begin{aligned}
\rho_{t}(x, y, t & +\Delta t)=-(\chi \rho(x, y, t+\Delta t) u(x, y, t+\Delta t))_{x}-(\chi \rho(x, y, t+\Delta t) v(x, y, t+\Delta t))_{y}+\Delta \rho(x, y, t+\Delta t) \\
& =\left(-\chi\left(\rho(x, y, t)+\rho_{t}(x, y, t) \Delta t+O\left(\Delta t^{2}\right)\right)\right)\left(u(x, y, t)+u_{t}(x, y, t) \Delta t+O\left(\Delta t^{2}\right)\right)_{x} \\
& +\left(-\chi\left(\rho(x, y, t)+\rho_{t}(x, y, t) \Delta t+O\left(\Delta t^{2}\right)\right)\right)\left(v(x, y, t)+v_{t}(x, y, t) \Delta t+O\left(\Delta t^{2}\right)\right)_{y} \\
& +\Delta\left(\rho(x, y, t)+\rho_{t}(x, y, t) \Delta t+O\left(\Delta t^{2}\right)\right)=-\left(\chi z_{\rho} z_{u}\right)_{x}-\left(\chi z_{\rho} z_{v}\right)_{y}+\Delta z_{\rho}+O\left(\Delta t^{2}\right)
\end{aligned}
$$

In the same way, it can be shown that

$$
c_{t}(x, y, t+\Delta t)=\Delta z_{c}-z_{c}+z_{\rho}+O\left(\Delta t^{2}\right)
$$

From (7.12) and the Taylor expansion above for $\rho$, it follows that:

$$
\begin{aligned}
& z_{\rho}^{i}=\rho^{i}-\left(\left(\chi \rho^{i} u^{i}\right)_{x}+\left(\chi \rho^{i} v^{i}\right)_{y}-\Delta \rho^{i}\right) \Delta t \\
& \rho^{i+1}=\frac{1}{2} \rho^{i}+\frac{1}{2} z_{\rho}^{i}-\left(\left(\chi z_{\rho}^{i} z_{u}^{i}\right)_{x}+\left(\chi z_{\rho}^{i} z_{v}^{i}\right)_{y}-\Delta z_{\rho}^{i}\right) \frac{\Delta t}{2}+O\left(\Delta t^{3}\right),
\end{aligned}
$$

Similarly, for $c$ we get:

$$
\begin{aligned}
& z_{c}^{i}=c^{i}-\left(-\Delta c^{i}+c^{i}-\rho^{i}\right) \Delta t \\
& c^{i+1}=\frac{1}{2} c^{i}+\frac{1}{2} z_{c}^{i}-\left(-\Delta z_{c}^{i}+z_{c}^{i}-z_{\rho}^{i}\right) \frac{\Delta t}{2}+O\left(\Delta t^{3}\right),
\end{aligned}
$$

Note that for $u$ and $v$ we will have:

$$
\begin{gathered}
z_{u}=u+u_{t} \Delta t=c_{x}+\left(c_{x}\right)_{t} \Delta t=\left(c+c_{t} \Delta t\right)_{x}=\left(z_{c}\right)_{x} \\
z_{v}=\left(z_{c}\right)_{y}
\end{gathered}
$$

Hence, it follows that:

$$
\begin{aligned}
& z_{u}^{i}=\left(z_{c}^{i}\right)_{x} \\
& u^{i+1}=\left(c^{i+1}\right)_{x},
\end{aligned}
$$




$$
\begin{aligned}
& z_{v}^{i}=\left(z_{c}^{i}\right)_{y} \\
& v^{i+1}=\left(c^{i+1}\right)_{y},
\end{aligned}
$$

Denoting by $\operatorname{Err}(x, y, i)=O\left(\Delta t^{3}\right)$ and multiplying the above equation (7.16)- (7.19) by the test functions $w_{\rho}, w_{c}, w_{u}$ and $w_{v}$, integrating by parts, and using consistency of the DG scheme we obtain the scheme for $z_{\rho}^{i}, \rho^{i+1}, z_{c}^{i}, c^{i+1}, z_{u}^{i}, u^{i+1}$ and $z_{v}^{i}, v^{i+1}$ :

$$
\begin{aligned}
& \int_{\Omega} z_{\rho}^{i} w^{\rho}=\int_{\Omega} \rho^{i} w_{\rho}+A_{\rho}\left(\rho^{i}, u^{i}, v^{i}, w^{\rho}\right) \Delta t \\
& \int_{\Omega} \rho^{i+1} w^{\rho}=\frac{1}{2} \int_{\Omega} \rho^{i} w^{\rho}+\frac{1}{2} \int_{\Omega} z_{\rho}^{i} w^{\rho}+A_{\rho}\left(z_{\rho}^{i}, z_{u}^{i}, z_{v}^{i}, w^{\rho}\right) \frac{\Delta t}{2}+\int_{\Omega} \operatorname{Err}(x, y, i) w^{\rho} \\
& \int_{\Omega} z_{c}^{i} w^{c}=\int_{\Omega} c^{i} w^{c}+A_{c}\left(c^{i}, \rho^{i}, w^{c}\right) \Delta t \\
& \int_{\Omega} c^{i+1} w^{c}=\frac{1}{2} \int_{\Omega} c^{i} w^{c}+\frac{1}{2} \int_{\Omega} z_{c}^{i} w^{c}+A_{c}\left(z_{\rho}^{i}, z_{c}^{i}, w^{c}\right) \frac{\Delta t}{2}+\int_{\Omega} \operatorname{Err}(x, y, i) w^{c} \\
& \int_{\Omega} z_{u}^{i} w^{u}+\sigma_{u} \sum_{e \in \Gamma_{h} \cup \partial \Omega_{\mathrm{ver}}} \frac{r_{u}^{2}}{|e|} \int_{e}\left[z_{u}^{i}\right]\left[w^{u}\right]=A_{u}\left(z_{c}^{i}, w^{u}\right), \\
& \int_{\Omega} u^{i+1} w^{u}+\sigma_{u} \sum_{e \in \Gamma_{h} \cup \partial \Omega_{\mathrm{ver}}} \frac{r_{u}^{2}}{|e|} \int_{e}\left[u^{i+1}\right]\left[w^{u}\right]=A_{u}\left(c^{i+1}, w^{u}\right) \\
& \int_{\Omega} z_{v}^{i} w^{v}+\sigma_{v} \sum_{e \in \Gamma_{h} \cup \partial \Omega_{\mathrm{hor}}} \frac{r_{v}^{2}}{|e|} \int_{e}\left[z_{v}^{i}\right]\left[w^{v}\right]=A_{v}\left(z_{c}^{i}, w^{v}\right), \\
& \int_{\Omega} v^{i+1} w^{v}+\sigma_{v} \sum_{e \in \Gamma_{h} \cup \partial \Omega_{\mathrm{hor}}} \frac{r_{v}^{2}}{|e|} \int_{e}\left[v^{i+1}\right]\left[w^{v}\right]=A_{v}\left(c^{i+1}, w^{v}\right),
\end{aligned}
$$

where we denoted by

$$
\begin{aligned}
& A_{\rho}\left(x_{1}, x_{2}, x_{3}, w^{\rho}\right):=-\left(\sum_{E \in \mathcal{E}_{h}} \int_{E} \nabla x_{1} \nabla w^{\rho}-\sum_{e \in \Gamma_{h}} \int_{e}\left\{\nabla x_{1} \cdot \mathbf{n}_{e}\right\}\left[w^{\rho}\right]+\varepsilon \sum_{e \in \Gamma_{h}} \int_{e}\left\{\nabla w^{\rho} \cdot \mathbf{n}_{e}\right\}\left[x_{1}\right]\right. \\
& \quad+\sigma_{\rho} \sum_{e \in \Gamma_{h}} \frac{r_{\rho}^{2}}{|e|} \int_{e}\left[x_{1}\right]\left[w^{\rho}\right]-\sum_{E \in \mathcal{E}_{h}} \int_{E} \chi x_{1} x_{2}\left(w^{\rho}\right)_{x}+\sum_{e \in \Gamma_{h}^{\text {ver }}} \int_{e}\left(\chi x_{1} x_{2}\right)^{*} n_{x}\left[w^{\rho}\right] \\
& \left.\quad-\sum_{E \in \mathcal{E}_{h}} \int_{E} \chi x_{1} x_{3}\left(w^{\rho}\right)_{y}+\sum_{e \in \Gamma_{h}^{\text {hor }}} \int_{e}\left(\chi x_{1} x_{3}\right)^{*} n_{y}\left[w^{\rho}\right]\right), \\
& A_{c}\left(x_{1}, x_{2}, w^{c}\right):=-\left(\sum_{E \in \mathcal{E}_{h}} \int_{E} \nabla x_{1} \nabla w^{c}-\sum_{e \in \Gamma_{h}} \int_{e}\left\{\nabla x_{1} \cdot \mathbf{n}_{e}\right\}\left[w^{c}\right]+\varepsilon \sum_{e \in \Gamma_{h}} \int_{e}\left\{\nabla w^{c} \cdot \mathbf{n}_{e}\right\}\left[x_{1}\right]\right. \\
& \left.\quad+\sigma_{c} \sum_{e \in \Gamma_{h}} \frac{r_{c}^{2}}{|e|} \int_{e}\left[x_{1}\right]\left[w^{c}\right]+\int_{\Omega} x_{1} w^{c}-\int_{\Omega} x_{2} w^{c}\right),
\end{aligned}
$$




$$
\begin{aligned}
& A_{u}\left(x_{1}, w^{u}\right):=-\left(\sum_{E \in \mathcal{E}_{h}} \int_{E} x_{1}\left(w^{u}\right)_{x}+\sum_{e \in \Gamma_{h}^{\mathrm{ver}}} \int_{e}\left(-x_{1}\right)_{u}^{*} n_{x}\left[w^{u}\right]-\sum_{e \in \partial \Omega_{\mathrm{ver}}} \int_{e} x_{1} n_{x} w^{u}\right), \\
& A_{v}\left(x_{1}, w^{v}\right):=-\left(\sum_{E \in \mathcal{E}_{h}} \int_{E} x_{1}\left(w^{v}\right)_{y}+\sum_{e \in \Gamma_{h}^{\mathrm{ver}}} \int_{e}\left(-x_{1}\right)_{v}^{*} n_{y}\left[w^{v}\right]-\sum_{e \in \partial \Omega_{\mathrm{ver}}} \int_{e} x_{1} n_{y} w^{v}\right),
\end{aligned}
$$

Let us again introduce similar notations to (6.14)

$$
\begin{aligned}
& \tau_{\rho}^{i}:=\rho_{\mathrm{DG}}^{i}-\widetilde{\rho}^{i}, \quad \xi_{\rho}^{i}:=\rho^{i}-\widetilde{\rho}^{i}, \quad \tau_{c}^{i}:=c_{\mathrm{DG}}^{i}-\widetilde{c}^{i}, \quad \xi_{c}^{i}:=c^{i}-\widetilde{c}^{i}, \\
& \tau_{u}^{i}:=u_{\mathrm{DG}}^{i}-\widetilde{u}^{i}, \quad \xi_{u}^{i}=u^{i}-\widetilde{u}^{i}, \quad \tau_{v}^{i}:=v_{\mathrm{DG}}^{i}-\widetilde{v}^{i}, \quad \xi_{v}^{i}:=v^{i}-\widetilde{v}^{i},
\end{aligned}
$$

and

$$
\begin{aligned}
& \tau_{z_{\rho}}^{i}:=z_{\mathrm{DG}, \rho}^{i}-\widetilde{z}_{\rho}^{i}, \quad \xi_{z_{\rho}}^{i}:=z_{\rho}^{i}-\widetilde{z}_{\rho}^{i}, \quad \tau_{z_{c}}^{i}:=z_{\mathrm{DG}, \mathrm{c}}^{i}-\widetilde{z}_{c}^{i}, \quad \xi_{z_{c}}^{i}:=z_{c}^{i}-\widetilde{z}_{c}^{i}, \\
& \tau_{z_{u}}^{i}:=z_{\mathrm{DG}, \mathrm{u}}^{i}-\widetilde{z}_{u}^{i}, \quad \xi_{z_{u}}^{i}=z_{u}^{i}-\widetilde{z}_{u}^{i}, \quad \tau_{z_{v}}^{i}:=z_{\mathrm{DG}, \mathrm{v}}^{i}-\widetilde{z}_{v}^{i}, \quad \xi_{z_{v}}^{i}:=z_{v}^{i}-\widetilde{z}_{v}^{i} .
\end{aligned}
$$

and subtract (7.20) from (7.1) and (7.5) respectively. We obtain the following error equations:

$$
\begin{aligned}
\int_{\Omega} \tau_{z_{\rho}}^{i} w^{\rho} & =\int_{\Omega} \tau_{\rho}^{i} w^{\rho}+M_{\rho}^{i}\left(w^{\rho}\right) \\
\int_{\Omega} \tau_{\rho}^{i+1} w^{\rho} & =\int_{\Omega}\left(\frac{1}{2} \tau_{\rho}^{i}+\frac{1}{2} \tau_{z_{\rho}}^{i}\right) w^{\rho}+\frac{1}{2} N_{\rho}^{i}\left(w^{\rho}\right) \\
& =\int_{\Omega} \tau_{\rho}^{i} w^{\rho}+\frac{1}{2} M_{\rho}^{i}\left(w^{\rho}\right)+\frac{1}{2} N_{\rho}^{i}\left(w^{\rho}\right)
\end{aligned}
$$

where

$$
\begin{aligned}
& M_{\rho}^{i}\left(w^{\rho}\right)=\int_{\Omega}\left(\xi_{z_{\rho}}^{i}-\xi_{\rho}^{i}\right) w^{\rho}+\left(A_{\rho}\left(\rho_{\mathrm{DG}}^{i}, u_{\mathrm{DG}}^{i}, v_{\mathrm{DG}}^{i}, w^{\rho}\right)-A_{\rho}\left(\rho^{i}, u^{i}, v^{i}, w^{\rho}\right)\right) \Delta t \\
& N_{\rho}^{i}\left(w^{\rho}\right)=\int_{\Omega}\left(2 \xi_{\rho}^{i+1}-\xi_{\rho}^{i}-\xi_{z_{\rho}}^{i}-2 E(x, y, i)\right) w^{\rho} \\
& +\left(A_{\rho}\left(z_{\mathrm{DG}, \rho}^{i}, z_{\mathrm{DG}, \mathrm{u}}^{i}, z_{\mathrm{DG}, \mathrm{v}}^{i}, w^{\rho}\right)-A_{\rho}\left(z_{\rho}^{i}, z_{u}^{i}, z_{v}^{i}, w^{\rho}\right)\right) \Delta t
\end{aligned}
$$

In the same way we obtain the error equations for concentration $c$, we obtain the following error equations:

$$
\begin{aligned}
\int_{\Omega} \tau_{z_{c}}^{i} w^{c} & =\int_{\Omega} \tau_{c}^{i} w^{c}+M_{c}^{i}\left(w^{c}\right) \\
\int_{\Omega} \tau_{c}^{i+1} w^{c} & =\int_{\Omega}\left(\frac{1}{2} \tau_{c}^{i}+\frac{1}{2} \tau_{z_{c}}^{i}\right) w^{c}+\frac{1}{2} N_{c}^{i}\left(w^{c}\right) \\
& =\int_{\Omega} \tau_{c}^{i} w^{c}+\frac{1}{2} M_{c}^{i}\left(w^{c}\right)+\frac{1}{2} N_{c}^{i}\left(w^{c}\right)
\end{aligned}
$$

where

$$
\begin{aligned}
& M_{c}^{i}\left(w^{c}\right)=\int_{\Omega}\left(\xi_{z_{c}}^{i}-\xi_{c}^{i}\right) w^{c}+\left(A_{c}\left(c_{\mathrm{DG}}^{i}, \rho_{\mathrm{DG}}^{i}, w^{\rho}\right)-A_{c}\left(c^{i}, \rho^{i}, w^{c}\right)\right) \Delta t \\
& N_{c}^{i}\left(w^{c}\right)=\int_{\Omega}\left(2 \xi_{c}^{i+1}-\xi_{c}^{i}-\xi_{z_{c}}^{i}-2 E(x, y, i)\right) w^{c}+\left(A_{c}\left(z_{\mathrm{DG}, c}^{i}, z_{\mathrm{DG}, \rho}^{i}, w^{c}\right)-A_{c}\left(z_{c}^{i}, z_{\rho}^{i}, w^{c}\right)\right)
\end{aligned}
$$


The error equations for $u$ and $v$ are :

$$
\begin{aligned}
& \int_{\Omega} \tau_{z_{u}}^{i} w^{u}+\sigma_{u} \sum_{e \in \Gamma_{h} \cup \partial \Omega_{\mathrm{ver}}} \frac{r_{u}^{2}}{|e|} \int_{e}\left[\tau_{z_{u}}^{i}\right]\left[w^{u}\right]=M_{u}^{i}\left(w^{u}\right), \\
& \int_{\Omega} \tau_{u}^{i+1} w^{u}+\sigma_{u} \sum_{e \in \Gamma_{h} \cup \partial \Omega_{\mathrm{ver}}} \frac{r_{u}^{2}}{|e|} \int_{e}\left[\tau_{u}^{i+1}\right]\left[w^{u}\right]=N_{u}^{i}\left(w^{u}\right)
\end{aligned}
$$

where

$$
\begin{gathered}
M_{u}^{i}\left(w^{u}\right)=\int_{\Omega} \xi_{z_{u}}^{i} w^{u}+\sigma_{u} \sum_{e \in \Gamma_{h} \cup \partial \Omega_{\mathrm{ver}}} \frac{r_{u}^{2}}{|e|} \int_{e}\left[\xi_{z_{u}}^{i}\right]\left[w^{u}\right]+\left(A_{u}\left(z_{\mathrm{DG}, \mathrm{c}}^{i}, w^{u}\right)-A_{u}\left(z_{c}^{i}, w^{u}\right)\right) \\
N_{u}^{i}\left(w^{u}\right)=\int_{\Omega} \xi_{u}^{i+1} w^{u}+\sigma_{u} \sum_{e \in \Gamma_{h} \cup \partial \Omega_{\mathrm{ver}}} \frac{r_{u}^{2}}{|e|} \int_{e}\left[\xi_{u}^{i+1}\right]\left[w^{u}\right]+\left(A_{u}\left(c_{\mathrm{DG}}^{i+1}, w^{u}\right)-A_{u}\left(c^{i+1}, w^{u}\right)\right) \\
\int_{\Omega} \tau_{z_{v}}^{i} w^{v}+\sigma_{v} \sum_{e \in \Gamma_{h} \cup \partial \Omega_{\mathrm{hor}}} \frac{r_{v}^{2}}{|e|} \int_{e}\left[\tau_{z_{v}}^{i}\right]\left[w^{v}\right]=M_{v}^{i}\left(w^{v}\right) \\
\int_{\Omega} \tau_{v}^{i+1} w^{v}+\sigma_{v} \sum_{e \in \Gamma_{h} \cup \partial \Omega_{\mathrm{hor}}} \frac{r_{v}^{2}}{|e|} \int_{e}\left[\tau_{v}^{i+1}\right]\left[w^{v}\right]=N_{v}^{i}\left(w^{v}\right)
\end{gathered}
$$

where

$$
\begin{aligned}
& M_{v}^{i}\left(w^{v}\right)=\int_{\Omega} \xi_{z_{v}}^{i} w^{v}+\sigma_{v} \sum_{e \in \Gamma_{h} \cup \partial \Omega_{\mathrm{hor}}} \frac{r_{v}^{2}}{|e|} \int_{e}\left[\xi_{z_{v}}^{i}\right]\left[w^{v}\right]+\left(A_{v}\left(z_{\mathrm{DG}, \mathrm{c}}^{i}, w^{v}\right)-A_{v}\left(z_{c}^{i}, w^{v}\right)\right) \\
& N_{u}^{i}\left(w^{u}\right)=\int_{\Omega} \xi_{v}^{i+1} w^{v}+\sigma_{v} \sum_{e \in \Gamma_{h} \cup \partial \Omega_{\mathrm{hor}}} \frac{r_{v}^{2}}{|e|} \int_{e}\left[\xi_{v}^{i+1}\right]\left[w^{v}\right]+\left(A_{v}\left(c_{\mathrm{DG}}^{i+1}, w^{v}\right)-A_{v}\left(c^{i+1}, w^{v}\right)\right)
\end{aligned}
$$

Next, set $w^{\rho}=\tau_{\rho}^{i}$ in the equation of (7.30) and $w^{\rho}=\tau_{z_{\rho}}^{i}$ in (7.31), add the two equations and after easy calculations, obtain the important equality that will be used to derive the error estimate for the density $\rho$ :

$$
\left\|\tau_{\rho}^{i+1}\right\|_{0, \Omega}^{2}-\left\|\tau_{\rho}^{i}\right\|_{0, \Omega}^{2}=\left\|\tau_{\rho}^{i+1}-\tau_{z_{\rho}}^{i}\right\|_{0, \Omega}^{2}+M_{\rho}^{i}\left(\tau_{\rho}^{i}\right)+N_{\rho}^{i}\left(\tau_{z_{\rho}}^{i}\right)
$$

Similarly, we obtain for the concentration $c$ :

$$
\left\|\tau_{c}^{i+1}\right\|_{0, \Omega}^{2}-\left\|\tau_{c}^{i}\right\|_{0, \Omega}^{2}=\left\|\tau_{c}^{i+1}-\tau_{z_{c}}^{i}\right\|_{0, \Omega}^{2}+M_{c}^{i}\left(\tau_{c}^{i}\right)+N_{c}^{i}\left(\tau_{z_{c}}^{i}\right)
$$

As for Forward Euler Scheme, let us make the following induction hypothesis:

$$
\begin{aligned}
& S R=\left\{\left(u_{\mathrm{DG}}^{i}, v_{\mathrm{DG}}^{i}\right) \in \mathcal{W}_{r_{u}, h}^{u} \times \mathcal{W}_{r_{v}, h}^{v}:\right. \\
& \sum_{i=0}^{n} \Delta t\left\|u_{\mathrm{DG}}^{i}-\widetilde{u}^{i}\right\|_{0, \Omega}^{2}
\end{aligned}
$$




$$
\begin{aligned}
& \leq C_{r u}\left(\frac{h^{2 \min \left(r_{\rho}+1, s_{\rho}\right)-2}}{r_{\rho}^{2 s_{\rho}-3}}+\frac{h^{2 \min \left(r_{c}+1, s_{c}\right)-2}}{r_{c}^{2 s_{c}-3}}+\frac{h^{2 \min \left(r_{u}+1, s_{u}\right)-2}}{r_{u}^{2 s_{u}-3}}+\frac{h^{2 \min \left(r_{v}+1, s_{v}\right)-2}}{r_{v}^{2 s_{v}-3}}\right) \\
& +C_{r u}^{t} \Delta t^{4}, \\
& \sum_{i=0}^{n} \Delta t\left\|v_{\mathrm{DG}}^{i}-\widetilde{v}^{i}\right\|_{0, \Omega}^{2} \\
& \leq C_{r v}\left(\frac{h^{2 \min \left(r_{\rho}+1, s_{\rho}\right)-2}}{r_{\rho}^{2 s_{\rho}-3}}+\frac{h^{2 \min \left(r_{c}+1, s_{c}\right)-2}}{r_{c}^{2 s_{c}-3}}+\frac{h^{2 \min \left(r_{u}+1, s_{u}\right)-2}}{r_{u}^{2 s_{u}-3}}+\frac{h^{2 \min \left(r_{v}+1, s_{v}\right)-2}}{r_{v}^{2 s_{v}-3}}\right) \\
& +C_{r v}^{t} \Delta t^{4}, \quad \sup _{0 \leq i \leq n}\left\|u_{\mathrm{DG}}^{i}-\widetilde{u}^{i}\right\|_{0, \Omega} \leq C_{r u}^{\star} \frac{h}{r_{\min }^{2}}, \sup _{0 \leq i \leq n}\left\|v_{\mathrm{DG}}^{i}-\widetilde{v}^{i}\right\|_{0, \Omega} \leq C_{r v}^{\star} \frac{h}{r_{\min }^{2}}, \\
& \left.\sup _{0 \leq i \leq n}\left\|\rho_{\mathrm{DG}}^{i}-\widetilde{\rho}^{i}\right\|_{0, \Omega} \leq C_{r \rho}^{\star} \frac{h^{2}}{r_{\min }^{4}}, \sup _{0 \leq i \leq n}\left\|c_{\mathrm{DG}}^{i}-\widetilde{c}^{i}\right\|_{0, \Omega} \leq C_{r c}^{\star} \frac{h^{2}}{r_{\min }^{4}}\right\},
\end{aligned}
$$

The induction hypothesis $S R$ implies the following lemma.

Lemma 7.1 For $\left(\rho_{\mathrm{DG}}^{i}, c_{\mathrm{DG}}^{i}, u_{\mathrm{DG}}^{i}, v_{\mathrm{DG}}^{i}\right) \in S R$, there exist positive constants $M_{\rho}, M_{c}, M_{u}, M_{v}$, $N_{\rho}, N_{c}, N_{u}$, and $N_{v}$ independent of $h, r_{\rho}, r_{c}, r_{u}$, and $r_{v}$, such that

$\sup _{0 \leq i \leq n}\left\|\rho_{\mathrm{DG}}^{i}\right\|_{\infty, \Omega} \leq M_{\rho}, \quad \sup _{0 \leq i \leq n}\left\|c_{\mathrm{DG}}^{i}\right\|_{\infty, \Omega} \leq M_{c}, \quad \sup _{0 \leq i \leq n}\left\|u_{\mathrm{DG}}^{i}\right\|_{\infty, \Omega} \leq M_{u}, \quad \sup _{0 \leq i \leq n}\left\|v_{\mathrm{DG}}^{i}\right\|_{\infty, \Omega} \leq M_{v}$,

$\sup _{0 \leq i \leq n}\left\|z_{\mathrm{DG}, \rho}^{i}\right\|_{\infty, \Omega} \leq N_{\rho}, \quad \sup _{0 \leq i \leq n}\left\|z_{\mathrm{DG}, \mathrm{c}}^{i}\right\|_{\infty, \Omega} \leq N_{c}, \quad \sup _{0 \leq i \leq n}\left\|z_{\mathrm{DG}, \mathrm{u}}^{i}\right\|_{\infty, \Omega} \leq N_{u}, \quad \sup _{0 \leq i \leq n}\left\|z_{\mathrm{DG}, \mathrm{v}}^{i}\right\|_{\infty, \Omega} \leq N_{v}$.

Proof: For the details of the proof see Appendix 9.

Theorem $7.2\left(l^{2}\left(H^{1}\right)\right.$ and $l^{\infty}\left(L^{2}\right)$ Runge-Kutta error estimates). Let the solution $\rho, c, u$ and $v$ of the Keller-Segel system (1.2) be sufficiently regular. Furthermore, we assume that penalty parameters $\sigma_{\rho}, \sigma_{c}, \sigma_{u}, \sigma_{v}$ are sufficiently large. Then the induction hypothesis holds true for $n+1$. Furthermore, there exists constants $C_{r \rho}$ and $C_{r c}$, independent of $h$ and $r$, such that

$$
\begin{aligned}
& \| \rho_{D G}- \rho\left\|_{l^{\infty}\left([0, T] ; L^{2}(\Omega)\right)}+\Delta t^{\frac{1}{2}}\right\| \mid \nabla\left(\rho_{D G}-\rho\right)\|\|_{l^{2}\left([0, T] ; L^{2}(\Omega)\right)}+\left(\sum_{i=0}^{N} \Delta t \sum_{e \in \Gamma_{h}} \frac{r_{\rho}^{2}}{|e|}\left\|\left[\rho_{D G}^{i}-\rho^{i}\right]\right\|_{0, e}^{2}\right)^{\frac{1}{2}} \\
& \leq C_{r \rho}\left(\frac{h^{\min \left(r_{\rho}+1, s_{\rho}\right)-1}}{r_{\rho}^{s_{\rho}-\frac{3}{2}}}+\frac{h^{\min \left(r_{c}+1, s_{c}\right)-1}}{r_{c}^{s_{c}-\frac{3}{2}}}+\frac{h^{\min \left(r_{u}+1, s_{u}\right)-1}}{r_{u}^{s_{u}-\frac{3}{2}}}+\frac{h^{\min \left(r_{v}+1, s_{v}\right)-1}}{r_{v}^{s_{v}-\frac{3}{2}}}+\Delta t^{2}\right) \\
&\left\|c_{D G}-c\right\|_{l^{\infty}\left([0, T] ; L^{2}(\Omega)\right)}+\Delta t^{\frac{1}{2}}\left\|\nabla\left(c_{D G}-c\right)\right\| l_{l^{2}\left([0, T] ; L^{2}(\Omega)\right)}+\left(\sum_{i=0}^{N} \Delta t \sum_{e \in \Gamma_{h}} \frac{r_{c}^{2}}{|e|}\left\|\left[c_{D G}^{i}-c^{i}\right]\right\|_{0, e}^{2}\right)^{\frac{1}{2}} \\
& \leq C_{r c}\left(\frac{h^{\min \left(r_{\rho}+1, s_{\rho}\right)-1}}{r_{\rho}^{s_{\rho}-\frac{3}{2}}}+\frac{h^{\min \left(r_{c}+1, s_{c}\right)-1}}{r_{c}^{s_{c}-\frac{3}{2}}}+\frac{h^{\min \left(r_{u}+1, s_{u}\right)-1}}{r_{u}^{s_{u}-\frac{3}{2}}}+\frac{h^{\min \left(r_{v}+1, s_{v}\right)-1}}{r_{v}^{s_{v}-\frac{3}{2}}}+\Delta t^{2}\right),
\end{aligned}
$$

where $\left(r_{\rho}, r_{c}, r_{u}, r_{v}\right) \geq 2$. 
The proof of the above theorem is postponed to Appendix 9.

Remark. The error estimates obtained in this paper are $h$-optimal and $r$-suboptimal (by $1 / 2)$, and the numerical tests reported in [18] confirm the theoretical error estimates.

\section{Numerical Example}

In this section, we consider the initial-boundary value problem for the Keller-Segel system and compare the solution obtained by the proposed Forward Euler DG method and by the RungeKutta DG method. We take the chemotactic sensitivity $\chi=1$ and the bell-shaped initial data

$$
\rho(x, y, 0)=1300 e^{-130\left(x^{2}+y^{2}\right)}, \quad c(x, y, 0)=650 e^{-65\left(x^{2}+y^{2}\right)} .
$$

We consider the above problem in the square domain $\left[-\frac{1}{2}, \frac{1}{2}\right] \times\left[-\frac{1}{2}, \frac{1}{2}\right]$. According to the results in [23], both components $\rho$ and $c$ of the solution are expected to blow up at the origin in finite time.

In Figures 8.1-8.4, we plot the contours of the density $\rho$ along the line $\mathcal{L}=\left[-\frac{1}{2}, \frac{1}{2}\right] \times 0$, computed at different times before blow-up on uniform grid with $h=1 / 101$ using quadratic polynomial approximation (Figures 8.1 and 8.2) and cubic polynomial approximation (Figures 8.3 and 8.4). The dashed line represents the solution obtained by the Forward Euler DG method and the solid line represents the solution obtained by the Runge-Kutta DG method. As one can see, the higher order time schemes are important for such type of problems with rapidly changing solution in time.

\section{Appendix: Proof of Several Estimates}

We collect in the Appendix details of the proofs of the several estimates.

\subsection{Derivation of the Estimate (6.37)}

To obtain the estimate (6.37), we again subtract (6.16) from (6.3) and choose $w^{\rho}=\tau_{\rho}^{i+1}-\tau_{\rho}^{i}$.

$$
\begin{gathered}
\left\|\tau_{\rho}^{i+1}-\tau_{\rho}^{i}\right\|_{0, \Omega}^{2}=-\Delta t \sum_{E \in \mathcal{E}_{h}} \int_{E} \nabla \tau_{\rho}^{i} \nabla\left(\tau_{\rho}^{i+1}-\tau_{\rho}^{i}\right) \\
+\Delta t \sum_{e \in \Gamma_{h}} \int_{e}\left\{\nabla \tau_{\rho}^{i} \cdot \mathbf{n}_{e}\right\}\left[\tau_{\rho}^{i+1}-\tau_{\rho}^{i}\right]-\varepsilon \Delta t \sum_{e \in \Gamma_{h}} \int_{e}\left\{\nabla\left(\tau_{\rho}^{i+1}-\tau_{\rho}^{i}\right) \cdot \mathbf{n}_{e}\right\}\left[\tau_{\rho}^{i}\right]-\Delta t \sigma_{\rho} \sum_{e \in \Gamma_{h}} \frac{r_{\rho}^{2}}{|e|} \int_{e}\left[\tau_{\rho}^{i}\right]\left[\tau_{\rho}^{i+1}-\tau_{\rho}^{i}\right] \\
+\Delta t \sum_{E \in \mathcal{E}_{h}} \int_{E} \chi \tau_{\rho}^{i} u_{\mathrm{DG}}^{i}\left(\tau_{\rho}^{i+1}-\tau_{\rho}^{i}\right)_{x}+\Delta t \sum_{E \in \mathcal{E}_{h}} \int_{E} \chi \widetilde{\rho}^{i} \tau_{u}^{i}\left(\tau_{\rho}^{i+1}-\tau_{\rho}^{i}\right)_{x} \\
-\Delta t \sum_{E \in \mathcal{E}_{h}} \int_{E} \chi \widetilde{\rho}^{i} \xi_{u}^{i}\left(\tau_{\rho}^{i+1}-\tau_{\rho}^{i}\right)_{x}-\Delta t \sum_{e \in \Gamma_{h}^{\mathrm{ver}}} \int_{e}\left(\left(\chi \rho_{\mathrm{DG}}^{i} u_{\mathrm{DG}}^{i}\right)^{*}-\left(\chi \rho^{i} u^{i}\right)^{* *}\right) n_{x}\left[\tau_{\rho}^{i+1}-\tau_{\rho}^{i}\right] \\
+\Delta t \sum_{E \in \mathcal{E}_{h}} \int_{E} \chi \tau_{\rho}^{i} v_{\mathrm{DG}}^{i}\left(\tau_{\rho}^{i+1}-\tau_{\rho}^{i}\right)_{y}+\Delta t \sum_{E \in \mathcal{E}_{h}} \int_{E} \chi \widetilde{\rho}^{i} \tau_{v}^{i}\left(\tau_{\rho}^{i+1}-\tau_{\rho}^{i}\right)_{y}-\Delta t \sum_{E \in \mathcal{E}_{h}} \int_{E} \chi \widetilde{\rho}^{i} \xi_{v}^{i}\left(\tau_{\rho}^{i+1}-\tau_{\rho}^{i}\right)_{y}
\end{gathered}
$$




$$
\begin{gathered}
-\Delta t \sum_{e \in \Gamma_{h}^{\text {hor }}} \int_{e}\left(\left(\chi \rho_{\mathrm{DG}}^{i} v_{\mathrm{DG}}^{i}\right)^{*}-\left(\chi \rho^{i} v^{i}\right)^{* *}\right) n_{y}\left[\tau_{\rho}^{i+1}-\tau_{\rho}^{i}\right]+\Delta t \int_{\Omega} \xi_{\rho t}\left(t^{i}\right)\left(\tau_{\rho}^{i+1}-\tau_{\rho}^{i}\right) \\
+\Delta t \int_{\Omega}\left(\widetilde{\rho}_{t}\left(t^{i}\right)-\frac{\widetilde{\rho}^{i+1}-\widetilde{\rho}^{i}}{\Delta t}\right)\left(\tau_{\rho}^{i+1}-\tau_{\rho}^{i}\right) \\
+\Delta t \sum_{E \in \mathcal{E}_{h}} \int_{E} \nabla \xi_{\rho}^{i} \nabla\left(\tau_{\rho}^{i+1}-\tau_{\rho}^{i}\right)-\Delta t \sum_{e \in \Gamma_{h}} \int_{e}\left\{\nabla \xi_{\rho}^{i} \cdot \mathbf{n}_{e}\right\}\left[\tau_{\rho}^{i+1}-\tau_{\rho}^{i}\right] \\
+\varepsilon t \sum_{e \in \Gamma_{h}} \int_{e}\left\{\nabla\left(\tau_{\rho}^{i+1}-\tau_{\rho}^{i}\right) \cdot \mathbf{n}_{e}\right\}\left[\xi_{\rho}^{i}\right]+\Delta t \sigma_{\rho} \sum_{e \in \Gamma_{h}} \frac{r_{\rho}^{2}}{|e|} \int_{e}\left[\xi_{\rho}^{i}\right]\left[\tau_{\rho}^{i+1}-\tau_{\rho}^{i}\right] \\
-\Delta t \sum_{E \in \mathcal{E}_{h}} \int_{E} \chi \xi_{\rho}^{i} u^{i}\left(\tau_{\rho}^{i+1}-\tau_{\rho}^{i}\right)_{x}-\Delta t \sum_{E \in \mathcal{E}_{h}} \int_{E} \chi \xi_{\rho}^{i} v^{i}\left(\tau_{\rho}^{i+1}-\tau_{\rho}^{i}\right)_{y} \\
=: T T_{1}^{\rho}+T T_{2}^{\rho}+\ldots+T T_{20}^{\rho} .
\end{gathered}
$$

Now let us bound each term on the RHS of (9.1). Using similar techniques as for the estimation of (6.17), except now we will use inverse inequality for the estimation of the $\nabla\left(\tau_{\rho}^{i+1}-\tau_{\rho}^{i}\right)$, and inequality (2.6) to estimate $\left\|\left[\tau_{\rho}^{i+1}-\tau_{\rho}^{i}\right]\right\|_{0, e}$. Hence we obtain the following estimates:

$$
\begin{gathered}
\left|T T_{1}^{\rho}\right| \leq \frac{1}{21}\left\|\tau_{\rho}^{i+1}-\tau_{\rho}^{i}\right\|_{0, \Omega}^{2}+R^{1} \frac{\Delta t^{2} r_{\rho}^{4}}{h^{2}}\left\|\mid \nabla \tau_{\rho}^{i}\right\| \|_{0, \Omega}^{2} \\
\left|T T_{2}^{\rho}\right| \leq \frac{1}{21}\left\|\tau_{\rho}^{i+1}-\tau_{\rho}^{i}\right\|_{0, \Omega}^{2}+R^{2} \frac{\Delta t^{2} r_{\rho}^{4}}{h^{2}}\left\|\left|\nabla \tau_{\rho}^{i}\right|\right\|_{0, \Omega}^{2} \\
\left|T T_{3}^{\rho}\right| \leq \frac{1}{21}\left\|\tau_{\rho}^{i+1}-\tau_{\rho}^{i}\right\|_{0, \Omega}^{2}+R^{3} \frac{\Delta t^{2} r_{\rho}^{4}}{h^{2}} \sum_{e \in \Gamma_{h}} \frac{r_{\rho}^{2}}{|e|}\left\|\left[\tau_{\rho}^{i}\right]\right\|_{0, e}^{2} \\
\left|T T_{4}^{\rho}\right| \leq \frac{1}{21}\left\|\tau_{\rho}^{i+1}-\tau_{\rho}^{i}\right\|_{0, \Omega}^{2}+R^{4} \frac{\Delta t^{2} r_{\rho}^{4}}{h^{2}} \sum_{e \in \Gamma_{h}} \frac{r_{\rho}^{2}}{|e|}\left\|\left[\tau_{\rho}^{i}\right]\right\|_{0, e}^{2} \\
\left|T T_{5}^{\rho}\right| \leq \frac{1}{21}\left\|\tau_{\rho}^{i+1}-\tau_{\rho}^{i}\right\|_{0, \Omega}^{2}+R^{5} \frac{\Delta t^{2} r_{\rho}^{4}}{h^{2}}\left\|\tau_{\rho}^{i}\right\|_{0, \Omega}^{2} \\
\left|T T_{6}^{\rho}\right| \leq \frac{1}{21}\left\|\tau_{\rho}^{i+1}-\tau_{\rho}^{i}\right\|_{0, \Omega}^{2}+R^{6} \frac{\Delta t^{2} r_{u}^{4}}{h^{2}}\left\|\tau_{u}^{i}\right\|_{0, \Omega}^{2} \\
\left|T T_{7}^{\rho}\right| \leq \frac{1}{21}\left\|\tau_{\rho}^{i+1}-\tau_{\rho}^{i}\right\|_{0, \Omega}^{2}+R^{7} \frac{\Delta t^{2} r_{\rho}^{4}}{h^{2}} \frac{h^{2} \min \left(r_{u}+1, s_{u}\right)}{r_{u}^{2 s_{u}}} \\
\left|T T_{8}^{\rho}\right| \leq \frac{1}{21}\left\|\tau_{\rho}^{i+1}-\tau_{\rho}^{i}\right\|_{0, \Omega}^{2}+R^{9} \frac{\Delta t^{2} r_{\rho}^{4}}{h^{2}}\left\|\tau_{\rho}^{i}\right\|_{0, \Omega}^{2}+R^{10} \frac{\Delta t^{2} r_{\rho}^{4}}{h^{2}}\left\|\tau_{u}^{i}\right\|_{0, \Omega}^{2} \\
+R^{11} \frac{\Delta t^{2} r_{\rho}^{4}}{h^{2}}\left(\frac{h^{2} \min \left(r_{\rho}+1, s_{\rho}\right)}{r_{\rho}^{2 s_{\rho}}}+\frac{h^{2} \min \left(r_{u}+1, s_{u}\right)}{r_{u}^{2 s_{u}}}\right) \\
\left|T T_{9}^{\rho}\right| \leq \frac{1}{21}\left\|\tau_{\rho}^{i+1}-\tau_{\rho}^{i}\right\|_{0, \Omega}^{2}+R^{12} \frac{\Delta t^{2} r_{\rho}^{4}}{h^{2}}\left\|\tau_{\rho}^{i}\right\|_{0, \Omega}^{2}
\end{gathered}
$$


Analysis of a DG Method for the Chemotaxis Model

$$
\begin{aligned}
& \left|T T_{10}^{\rho}\right| \leq \frac{1}{21}\left\|\tau_{\rho}^{i+1}-\tau_{\rho}^{i}\right\|_{0, \Omega}^{2}+R^{13} \frac{\Delta t^{2} r_{v}^{4}}{h^{2}}\left\|\tau_{v}^{i}\right\|_{0, \Omega}^{2} \\
& \left|T T_{11}^{\rho}\right| \leq \frac{1}{21}\left\|\tau_{\rho}^{i+1}-\tau_{\rho}^{i}\right\|_{0, \Omega}^{2}+R^{14} \frac{\Delta t^{2} r_{\rho}^{4}}{h^{2}} \frac{h^{2 \min \left(r_{v}+1, s_{v}\right)}}{r_{v}^{2 s_{v}}} \\
& \left|T T_{12}^{\rho}\right| \leq \frac{1}{21}\left\|\tau_{\rho}^{i+1}-\tau_{\rho}^{i}\right\|_{0, \Omega}^{2}+R^{16} \frac{\Delta t^{2} r_{\rho}^{4}}{h^{2}}\left\|\tau_{\rho}^{i}\right\|_{0, \Omega}^{2}+R^{17} \frac{\Delta t^{2} r_{\rho}^{4}}{h^{2}}\left\|\tau_{v}^{i}\right\|_{0, \Omega}^{2} \\
& +R^{18} \frac{\Delta t^{2} r_{\rho}^{4}}{h^{2}}\left(\frac{h^{2 \min \left(r_{\rho}+1, s_{\rho}\right)}}{r_{\rho}^{2 s_{\rho}}}+\frac{h^{2 \min \left(r_{v}+1, s_{v}\right)}}{r_{v}^{2 s_{v}}}\right) . \\
& \left|T T_{13}^{\rho}\right| \leq \frac{1}{21}\left\|\tau_{\rho}^{i+1}-\tau_{\rho}^{i}\right\|_{0, \Omega}^{2}+R^{19} \Delta t^{2} \frac{h^{2 \min \left(r_{\rho}+1, s_{\rho}\right)}}{r_{\rho}^{2 s_{\rho}}} \\
& \left|T T_{14}^{\rho}\right| \leq \frac{1}{21}\left\|\tau_{\rho}^{i+1}-\tau_{\rho}^{i}\right\|_{0, \Omega}^{2}+R^{20} \Delta t^{3} \int_{t^{i}}^{t^{i+1}}\left\|\partial_{t t} \widetilde{\rho}(s)\right\|_{0, \Omega}^{2} \mathrm{ds} . \\
& \left|T T_{15}^{\rho}\right| \leq \frac{1}{21}\left\|\tau_{\rho}^{i+1}-\tau_{\rho}^{i}\right\|_{0, \Omega}^{2}+R^{21} \frac{\Delta t^{2} r_{\rho}^{4}}{h^{2}} \frac{h^{2 \min \left(r_{\rho}+1, s_{\rho}\right)-2}}{r_{\rho}^{2 s_{\rho}-2}} \\
& \left|T T_{16}^{\rho}\right| \leq \frac{1}{21}\left\|\tau_{\rho}^{i+1}-\tau_{\rho}^{i}\right\|_{0, \Omega}^{2}+R^{22} \frac{\Delta t^{2} r_{\rho}^{4}}{h^{2}} \frac{h^{2 \min \left(r_{\rho}+1, s_{\rho}\right)-2}}{r_{\rho}^{2 s_{\rho}-2}} \\
& \left|T T_{17}^{\rho}\right| \leq \frac{1}{21}\left\|\tau_{\rho}^{i+1}-\tau_{\rho}^{i}\right\|_{0, \Omega}^{2}+R^{23} \frac{\Delta t^{2} r_{\rho}^{4}}{h^{2}} \frac{h^{2 \min \left(r_{\rho}+1, s_{\rho}\right)-2}}{r_{\rho}^{2 s_{\rho}-3}} \\
& \left|T T_{18}^{\rho}\right| \leq \frac{1}{21}\left\|\tau_{\rho}^{i+1}-\tau_{\rho}^{i}\right\|_{0, \Omega}^{2}+R^{24} \frac{\Delta t^{2} r_{\rho}^{4}}{h^{2}} \frac{h^{2 \min \left(r_{\rho}+1, s_{\rho}\right)-2}}{r_{\rho}^{2 s_{\rho}-3}} \\
& \left|T T_{19}^{\rho}\right| \leq \frac{1}{21}\left\|\tau_{\rho}^{i+1}-\tau_{\rho}^{i}\right\|_{0, \Omega}^{2}+R^{25} \frac{\Delta t^{2} r_{\rho}^{4}}{h^{2}} \frac{h^{2 \min \left(r_{\rho}+1, s_{\rho}\right)}}{r_{\rho}^{2 s_{\rho}}} \\
& \left|T T_{20}^{\rho}\right| \leq \frac{1}{21}\left\|\tau_{\rho}^{i+1}-\tau_{\rho}^{i}\right\|_{0, \Omega}^{2}+R^{26} \frac{\Delta t^{2} r_{\rho}^{4}}{h^{2}} \frac{h^{2 \min \left(r_{\rho}+1, s_{\rho}\right)}}{r_{\rho}^{2 s_{\rho}}}
\end{aligned}
$$

Now, combining all the bounds (9.2)-(9.21) and using the assumption that $h<1, r>1$, the estimate (6.37) follows.

\subsection{Derivation of the Estimate for the Concentration (6.44)}

First, from the consistency Lemma 4.1 we obtain that the exact solution of (1.2) satisfying the weak formulation of the form of the equation (3.4), which may be rewritten as

$$
\begin{aligned}
& \int_{\Omega} \widetilde{c}_{t}\left(t^{i}\right) w^{c}+\sum_{E \in \mathcal{E}_{h}} \int_{E} \nabla \widetilde{c}^{i} \nabla w^{c}-\sum_{e \in \Gamma_{h}} \int_{e}\left\{\nabla \widetilde{c}^{i} \cdot \mathbf{n}_{e}\right\}\left[w^{c}\right]+\varepsilon \sum_{e \in \Gamma_{h}} \int_{e}\left\{\nabla w^{c} \cdot \mathbf{n}_{e}\right\}\left[\widetilde{c}^{i}\right]+\sigma_{c} \sum_{e \in \Gamma_{h}} \frac{r_{c}^{2}}{|e|} \int_{e}\left[\widetilde{c}^{i}\right]\left[w^{c}\right] \\
& \quad+\int_{\Omega} \widetilde{c}^{i} w^{c}-\int_{\Omega} \widetilde{\rho}^{i} w^{c}=-\int_{\Omega} \xi_{c t}\left(t^{i}\right) w^{c}-\sum_{E \in \mathcal{E}_{h}} \int_{E} \nabla \xi_{c}^{i} \nabla w^{c}+\sum_{e \in \Gamma_{h}} \int_{e}\left\{\nabla \xi_{c}^{i} \cdot \mathbf{n}_{e}\right\}\left[w^{c}\right] \\
& \quad-\varepsilon \sum_{e \in \Gamma_{h}} \int_{e}\left\{\nabla w^{c} \cdot \mathbf{n}_{e}\right\}\left[\xi_{c}^{i}\right]-\sigma_{c} \sum_{e \in \Gamma_{h}} \frac{r_{c}^{2}}{|e|} \int_{e}\left[\xi_{c}^{i}\right]\left[w^{c}\right]-\int_{\Omega} \xi_{c}^{i} w^{c}+\int_{\Omega} \xi_{\rho}^{i} w^{c} .
\end{aligned}
$$


We then subtract equation (9.22) from equation (6.4) and set $w^{c}=\tau_{c}^{i}$ to obtain

$$
\begin{aligned}
& \left\|\tau_{c}^{i+1}\right\|_{0, \Omega}^{2}-\left\|\tau_{c}^{i}\right\|_{0, \Omega}^{2}+2 \Delta t\left|\left\|\nabla \tau_{c}^{i} \mid\right\|_{0, \Omega}^{2}+2 \Delta t \sigma_{\rho} \sum_{e \in \Gamma_{h}} \frac{r_{c}^{2}}{|e|}\left\|\left[\tau_{c}^{i}\right]\right\|_{0, e}^{2}=\left\|\tau_{c}^{i+1}-\tau_{c}^{i}\right\|_{0, \Omega}^{2}\right. \\
& \quad+2 \Delta t \int_{\Omega} \xi_{c}^{i} \tau_{c}^{i}-2 \Delta t \int_{\Omega} \xi_{\rho}^{i} \tau_{c}^{i}-2 \Delta t \int_{\Omega} \tau_{\rho}^{i} \tau_{c}^{i}-2 \Delta t\left\|\tau_{c}^{i}\right\|_{0, \Omega}^{2}+2 \Delta t \int_{\Omega}\left(\widetilde{c}_{t}\left(t^{i}\right)-\frac{\widetilde{c}^{i+1}-\widetilde{c}^{i}}{\Delta t}\right) \tau_{c}^{i} \\
& \quad+2 \Delta t(1-\varepsilon) \sum_{e \in \Gamma_{h}} \int_{e}\left\{\nabla \tau_{c}^{i} \cdot \mathbf{n}_{e}\right\}\left[\tau_{c}^{i}\right]+2 \Delta t \int_{\Omega} \xi_{c t}\left(t^{i}\right) \tau_{c}^{i}+2 \Delta t \sum_{E \in \mathcal{E}_{h}} \int_{E} \nabla \xi_{c}^{i} \nabla \tau_{c}^{i}-2 \Delta t \sum_{e \in \Gamma_{h}} \iint_{e}\left\{\nabla \xi_{c}^{i} \cdot \mathbf{n}_{e}\right\}\left[\tau_{c}^{i}\right] \\
& \quad+2 \Delta t \varepsilon \sum_{e \in \Gamma_{h}} \int_{e}\left\{\nabla \tau_{c}^{i} \cdot \mathbf{n}_{e}\right\}\left[\xi_{c}^{i}\right]+2 \Delta t \sigma_{c} \sum_{e \in \Gamma_{h}} \frac{r_{c}^{2}}{|e|} \int_{e}\left[\xi_{c}^{i}\right]\left[\tau_{c}^{i}\right]=T_{1}^{c}+T_{c}^{2}+\ldots+T_{c}^{12}
\end{aligned}
$$

Let us notice again that the term on the LHS of (9.23) is rewritten similar to the term on the LHS of (6.17):

$$
\int_{\Omega} \frac{\tau_{c}^{i+1}-\tau_{c}^{i}}{\Delta t} \tau_{c}^{i}=\frac{\left\|\tau_{c}^{i+1}\right\|_{0, \Omega}^{2}}{2 \Delta t}-\frac{\left\|\tau_{c}^{i}\right\|_{0, \Omega}^{2}}{2 \Delta t}-\frac{\left\|\tau_{c}^{i+1}-\tau_{c}^{i}\right\|_{0, \Omega}^{2}}{2 \Delta t}
$$

The terms on the RHS of (9.23) are bounded using the same techniques as in (6.17). As before, we start with term $T_{2}^{c}$ :

$$
\begin{gathered}
\left|T_{2}^{c}\right| \leq \varepsilon_{2}^{c} \Delta t\left\|\tau_{c}^{i}\right\|_{0, \Omega}^{2}+C_{2}^{c} \Delta t \frac{h^{2 \min \left(r_{c}+1, s_{c}\right)}}{r_{c}^{2 s_{c}}} \\
\left|T_{3}^{c}\right| \leq \varepsilon_{3}^{c} \Delta t\left\|\tau_{c}^{i}\right\|_{0, \Omega}^{2}+C_{3}^{c} \Delta t \frac{h^{2 \min \left(r_{\rho}+1, s_{\rho}\right)}}{r_{\rho}^{2 s_{\rho}}} \\
\left|T_{4}^{c}\right| \leq \varepsilon_{4}^{c} \Delta t\left\|\tau_{c}^{i}\right\|_{0, \Omega}^{2}+C_{4}^{c} \Delta t\left\|\tau_{\rho}^{i}\right\|_{0, \Omega}^{2} \\
\left|T_{5}^{c}\right|=2 \Delta t\left\|\tau_{c}^{i}\right\|_{0, \Omega}^{2} \\
\left|T_{6}^{c}\right| \leq \varepsilon_{6}^{c} \Delta t\left\|\tau_{c}^{i}\right\|_{0, \Omega}^{2}+C_{6}^{c} \Delta t^{2} \int_{t^{i}}^{t^{i+1}}\left\|\partial_{t t} \widetilde{c}(s)\right\|_{0, \Omega}^{2} \mathrm{ds} \\
\left|T_{7}^{c}\right| \leq \varepsilon_{7}^{c} \Delta t\left|\left\|\left.\nabla \tau_{c}^{i}||\right|_{0, \Omega} ^{2}+C_{7}^{c} \Delta t \frac{r_{c}^{2}}{|e|} \sum_{e \in \Gamma_{h}}\right\|\left[\tau_{c}^{i}\right] \|_{0, e}^{2}\right. \\
\left|T_{8}^{c}\right| \leq \varepsilon_{8}^{c} \Delta t\left\|\tau_{c}^{i}\right\|_{0, \Omega}^{2}+C_{8}^{c} \Delta t \frac{h^{2 \min \left(r_{c}+1, s_{c}\right)}}{r_{c}^{2 s_{c}}} \\
\left|T_{9}^{c}\right| \leq \varepsilon_{9}^{c} \Delta t \mid\left\|\nabla \tau_{c}^{i}\right\| \|_{0, \Omega}^{2}+C_{9}^{c} \Delta t \frac{h^{2 \min \left(r_{c}+1, s_{c}\right)-2}}{r_{c}^{2 s_{c}-2}} \\
\left|T_{10}^{c}\right| \leq \varepsilon_{10}^{c} \Delta t \sum_{e \in \Gamma_{h}} \frac{r_{c}^{2}}{|e|}\left\|\left[\tau_{c}^{i}\right]\right\|_{0, \Omega}^{2}+C_{10}^{c} \Delta t \frac{h^{2 \min \left(r_{c}+1, s_{c}\right)-2}}{r_{c}^{2 s_{c}-2}} \\
\left|T_{11}^{c}\right| \leq \varepsilon_{11}^{c} \Delta t\left|\left\|\nabla \tau_{c}^{i}\right\|\right|_{0, \Omega}^{2}+C_{11}^{c} \Delta t \frac{h^{2 \min \left(r_{c}+1, s_{c}\right)-2}}{r_{c}^{2 s_{c}-3}}
\end{gathered}
$$




$$
\left|T_{12}^{c}\right| \leq \varepsilon_{12}^{c} \Delta t \sum_{e \in \Gamma_{h}} \frac{r_{c}^{2}}{|e|}\left\|\left[\tau_{c}^{i}\right]\right\|_{0, e}^{2}+C_{12}^{c} \Delta t \frac{h^{2 \min \left(r_{c}+1, s_{c}\right)-2}}{r_{c}^{2 s_{c}-3}}
$$

Next, we need to bound the term $T_{1}^{c}$ as it was done for the $T_{1}^{\rho}$. We subtract (9.22) from equation (6.4) and set $w^{c}=\tau_{c}^{i+1}-\tau_{c}^{i}$ to obtain:

$$
\begin{aligned}
& \left\|\tau_{c}^{i+1}-\tau_{c}^{i}\right\|_{0, \Omega}^{2}=\Delta t \int_{\Omega} \xi_{c}^{i}\left(\tau_{c}^{i+1}-\tau_{c}^{i}\right)-\Delta t \int_{\Omega} \xi_{\rho}^{i}\left(\tau_{c}^{i+1}-\tau_{c}^{i}\right)-\Delta t \int_{\Omega} \tau_{\rho}^{i}\left(\tau_{c}^{i+1}-\tau_{c}^{i}\right)-\Delta t \int_{\Omega} \tau_{c}^{i}\left(\tau_{c}^{i+1}-\tau_{c}^{i}\right) \\
& +\Delta t \int_{\Omega}\left(\widetilde{c}_{t}\left(t^{i}\right)-\frac{\widetilde{c}^{i+1}-\widetilde{c}^{i}}{\Delta t}\right)\left(\tau_{c}^{i+1}-\tau_{c}^{i}\right)-\Delta t \sum_{E \in \mathcal{E}_{h}} \int_{E} \nabla \tau_{c}^{i} \nabla\left(\tau_{c}^{i+1}-\tau_{c}^{i}\right)+\Delta t \sum_{e \in \Gamma_{h}} \int_{e}\left\{\nabla \tau_{c}^{i} \cdot \mathbf{n}_{e}\right\}\left[\tau_{c}^{i+1}-\tau_{c}^{i}\right] \\
& \quad-\varepsilon \Delta t \sum_{e \in \Gamma_{h}} \int_{e}\left\{\nabla\left(\tau_{c}^{i+1}-\tau_{c}^{i}\right) \cdot \mathbf{n}_{e}\right\}\left[\tau_{c}^{i}\right]-\Delta t \sigma_{c} \sum_{e \in \Gamma_{h}} \frac{r_{c}^{2}}{|e|} \int_{e}\left[\tau_{c}^{i}\right]\left[\tau_{c}^{i+1}-\tau_{c}^{i}\right]+\Delta t \int_{\Omega} \xi_{c t}\left(t^{i}\right)\left(\tau_{c}^{i+1}-\tau_{c}^{i}\right) \\
& +\Delta t \sum_{E \in \mathcal{E}_{h}} \int_{E} \nabla \xi_{c}^{i} \nabla\left(\tau_{c}^{i+1}-\tau_{c}^{i}\right)-\Delta t \sum_{e \in \Gamma_{h}} \int_{e}\left\{\nabla \xi_{c}^{i} \cdot \mathbf{n}_{e}\right\}\left[\tau_{c}^{i+1}-\tau_{c}^{i}\right]+\Delta t \varepsilon \sum_{e \in \Gamma_{h}} \int_{e}\left\{\nabla\left(\tau_{c}^{i+1}-\tau_{c}^{i}\right) \cdot \mathbf{n}_{e}\right\}\left[\xi_{c}^{i}\right] \\
& +\Delta t \sigma_{c} \sum_{e \in \Gamma_{h}} \frac{r_{c}^{2}}{|e|} \int_{e}\left[\xi_{c}^{i}\right]\left[\tau_{c}^{i+1}-\tau_{c}^{i}\right]=T T_{1}^{c}+T T_{c}^{2}+\ldots+T T_{c}^{14} .
\end{aligned}
$$

Using similar techniques as in (9.1), we obtain the following estimates:

$$
\begin{aligned}
& \left|T T_{1}^{c}\right| \leq \frac{1}{15}\left\|\tau_{c}^{i+1}-\tau_{c}^{i}\right\|_{0, \Omega}^{2}+\Delta t^{2} K C^{1} \frac{h^{2 \min \left(r_{c}+1, s_{c}\right)}}{r_{c}^{2 s_{c}}} \\
& \left|T T_{2}^{c}\right| \leq \frac{1}{15}\left\|\tau_{c}^{i+1}-\tau_{c}^{i}\right\|_{0, \Omega}^{2}+\Delta t^{2} K C^{2} \frac{h^{2 \min \left(r_{\rho}+1, s_{\rho}\right)}}{r_{\rho}^{2 s_{\rho}}} \\
& \left|T T_{3}^{c}\right| \leq \frac{1}{15}\left\|\tau_{c}^{i+1}-\tau_{c}^{i}\right\|_{0, \Omega}^{2}+\Delta t^{2} K C^{3}\left\|\tau_{\rho}^{i}\right\|_{0, \Omega}^{2} \\
& \left|T T_{4}^{c}\right| \leq \frac{1}{15}\left\|\tau_{c}^{i+1}-\tau_{c}^{i}\right\|_{0, \Omega}^{2}+\Delta t^{2} K C^{4}\left\|\tau_{c}^{i}\right\|_{0, \Omega}^{2} \\
& \left|T T_{5}^{c}\right| \leq \frac{1}{15}\left\|\tau_{c}^{i+1}-\tau_{c}^{i}\right\|_{0, \Omega}^{2}+K C^{5} \Delta t^{3} \int_{t^{i}}^{t^{i+1}}\left\|\partial_{t t} \widetilde{c}(s)\right\|_{0, \Omega}^{2} \mathrm{ds} . \\
& \left|T T_{6}^{c}\right| \leq \frac{1}{15}\left\|\tau_{c}^{i+1}-\tau_{c}^{i}\right\|_{0, \Omega}^{2}+K C^{6} \frac{\Delta t^{2} r_{c}^{4}}{h^{2}}|| \nabla \tau_{c}^{i} \|\left.\right|_{0, \Omega} ^{2} \\
& \left|T T_{7}^{c}\right| \leq \frac{1}{15}\left\|\tau_{c}^{i+1}-\tau_{c}^{i}\right\|_{0, \Omega}^{2}+\left.K C^{7} \frac{\Delta t^{2} r_{c}^{4}}{h^{2}}||\left|\nabla \tau_{c}^{i}\right|\right|_{0, \Omega} ^{2} \\
& \left|T T_{8}^{c}\right| \leq \frac{1}{15}\left\|\tau_{c}^{i+1}-\tau_{c}^{i}\right\|_{0, \Omega}^{2}+K C^{8} \frac{\Delta t^{2} r_{c}^{4}}{h^{2}} \sum_{e \in \Gamma_{h}} \frac{r_{c}^{2}}{|e|}\left\|\left[\tau_{c}^{i}\right]\right\|_{0, e}^{2} \\
& \left|T T_{9}^{c}\right| \leq \frac{1}{15}\left\|\tau_{c}^{i+1}-\tau_{c}^{i}\right\|_{0, \Omega}^{2}+K C^{9} \frac{\Delta t^{2} r_{c}^{4}}{h^{2}} \sum_{e \in \Gamma_{h}} \frac{r_{c}^{2}}{|e|}\left\|\left[\tau_{c}^{i}\right]\right\|_{0, e}^{2} \\
& \left|T T_{10}^{c}\right| \leq \frac{1}{15}\left\|\tau_{c}^{i+1}-\tau_{c}^{i}\right\|_{0, \Omega}^{2}+\Delta t^{2} K C^{10} \frac{h^{2 \min \left(r_{c}+1, s_{c}\right)}}{r_{c}^{2 s_{c}}}
\end{aligned}
$$




$$
\begin{aligned}
\left|T T_{11}^{c}\right| & \leq \frac{1}{15}\left\|\tau_{c}^{i+1}-\tau_{c}^{i}\right\|_{0, \Omega}^{2}+K C^{11} \frac{\Delta t^{2} r_{c}^{4}}{h^{2}} \frac{h^{2 \min \left(r_{c}+1, s_{c}\right)-2}}{r_{c}^{2 s_{c}-2}} \\
\left|T T_{12}^{c}\right| & \leq \frac{1}{15}\left\|\tau_{c}^{i+1}-\tau_{c}^{i}\right\|_{0, \Omega}^{2}+K C^{12} \frac{\Delta t^{2} r_{c}^{4}}{h^{2}} \frac{h^{2 \min \left(r_{c}+1, s_{c}\right)-2}}{r_{c}^{2 s_{c}-2}} \\
\left|T T_{13}^{c}\right| & \leq \frac{1}{15}\left\|\tau_{c}^{i+1}-\tau_{c}^{i}\right\|_{0, \Omega}^{2}+K C^{13} \frac{\Delta t^{2} r_{c}^{4}}{h^{2}} \frac{h^{2 \min \left(r_{c}+1, s_{c}\right)-2}}{r_{c}^{2 s_{c}-3}} \\
\left|T T_{14}^{c}\right| & \leq \frac{1}{15}\left\|\tau_{c}^{i+1}-\tau_{c}^{i}\right\|_{0, \Omega}^{2}+K C^{14} \frac{\Delta t^{2} r_{c}^{4}}{h^{2}} \frac{h^{2 \min \left(r_{c}+1, s_{c}\right)-2}}{r_{c}^{2 s_{c}-3}}
\end{aligned}
$$

We combine all the bounds (9.25)-(9.35) and use bounds (9.37)-(9.50) to estimate $\left\|\tau_{c}^{i+1}-\tau_{c}^{i}\right\|_{0, \Omega}^{2}$ (similar to (6.37)). Then we plug the estimates in (9.23). Choosing $\Delta t \leq C \frac{h^{2}}{r_{c}^{4}}$, summing for $i=0, . ., n$, applying Lemma 2.4 for $\left\|\tau_{\rho}^{i}\right\|_{0, \Omega}$, using estimate (6.43) and applying discrete Gronwall's lemma we obtain the final estimate for the $\tau_{c}^{i+1}$ :

$$
\begin{gathered}
\left\|\tau_{c}^{n+1}\right\|_{0, \Omega}^{2}+\sum_{i=0}^{n} \Delta t\left(\left\|\mid \nabla \tau_{c}^{i}\right\|\left\|_{0, \Omega}^{2}+\sum_{e \in \Gamma_{h}} \frac{r_{c}^{2}}{|e|}\right\|\left[\tau_{c}^{i}\right] \|_{0, e}^{2}\right) \leq C^{c}\left(\frac{h^{2 \min \left(r_{\rho}+1, s_{\rho}\right)-2}}{r_{\rho}^{2 s_{\rho}-3}}+\frac{h^{2 \min \left(r_{c}+1, s_{c}\right)-2}}{r_{c}^{2 s_{c}-3}}\right. \\
\left.+\frac{h^{2 \min \left(r_{u}+1, s_{u}\right)-2}}{r_{u}^{2 s_{u}-3}}+\frac{h^{2 \min \left(r_{v}+1, s_{v}\right)-2}}{r_{v}^{2 s_{v}-3}}\right)+\widetilde{C}_{c}^{t} \Delta t^{2}
\end{gathered}
$$

\subsection{Proof of the Induction Hypothesis (6.12) for $u_{\mathrm{DG}}^{n+1}$}

Let us consider (6.46) and bound each term on the RHS of (6.46).

Consider the term $T_{1}^{u}$. Using inverse inequality, Cauchy-Schwarz, assumption (3.2) and Young's inequality, we obtain:

$$
\left|T_{1}^{u}\right| \leq \varepsilon_{1}^{u}\left\|\tau_{u}^{i+1}\right\|_{0, \Omega}^{2}+U U_{1} \frac{r_{c}^{4}}{h^{2}}\left\|\tau_{c}^{i+1}\right\|_{0, \Omega}^{2}
$$

A bound for $T_{2}^{u}$ can be obtained in a similar way to the bound on $T_{8}^{\rho}$ :

$$
\begin{aligned}
\left|T_{2}^{u}\right| & \leq \sum_{e \in \Gamma_{h}^{\text {ver }}}\left(\left|\int_{e} \frac{a_{i}^{\text {out }}}{a_{i}^{\text {out }}-a_{i}^{\text {in }}}\left(\left(c_{\mathrm{DG}}^{i+1}\right)_{e}^{E^{1}}-\left(c^{i+1}\right)_{e}^{E^{1}}\right) n_{x}\left[\tau_{u}^{i+1}\right]\right|+\mid \int_{e} \frac{-a_{i}^{\text {in }}}{a_{i}^{\text {out }}-a_{i}^{\text {in }}}\left(\left(c_{\mathrm{DG}}^{i+1}\right)_{e}^{E^{2}}-\left(c^{i+1}\right)_{e}^{E^{2}}\right) n_{x}\left[\tau_{u}^{i+1}\right]\right. \\
& \left.+\left|\int_{e} \frac{-a_{i}^{\text {in }} a_{i}^{\text {out }}}{a_{i}^{\text {out }}-a_{i}^{\text {in }}}\left[u_{\mathrm{DG}}^{i+1}-u^{i+1}\right] n_{x}\left[\tau_{u}^{i+1}\right]\right|\right):=\mathrm{I}+\mathrm{II}+\mathrm{III} .
\end{aligned}
$$

From (6.8) and (6.14), the first term on the RHS of (6.46) can be estimated by

$$
\mathrm{I} \leq \sum_{e \in \Gamma_{h}}\left(\left|\int_{e}\left(\tau_{c}^{i+1}\right)_{e}^{E^{1}} n_{x}\left[\tau_{u}^{i+1}\right]\right|+\left|\int_{e}\left(\xi_{c}^{i+1}\right)_{e}^{E^{1}} n_{x}\left[\tau_{u}^{i+1}\right]\right|\right):=\widetilde{\mathrm{I}} .
$$

Then, using the Cauchy-Schwarz inequality, the trace inequality (2.4), the inequality (2.6), and the assumption (3.2), we estimate $\widetilde{\mathrm{I}}$ as follows:

$$
\widetilde{\mathrm{I}} \leq K U_{1}\left\|\tau_{c}^{i+1}\right\|_{0, \Omega}^{2}+K U_{2} \sum_{e \in \Gamma_{h}} \frac{r_{u}^{2}}{|e|}\left\|\left[\tau_{u}^{i+1}\right]\right\|_{0, e}^{2}+K U_{3} \frac{h^{2 \min \left(r_{c}+1, s_{c}\right)}}{r_{c}^{2 s_{c}}} .
$$


A similar bound can be derived for the second term on the RHS of (6.46). The third term on the RHS of (6.46) is similar to the third term on the RHS of (6.23), hence it can be bounded by

$$
\mathrm{III} \leq\left(\frac{K U_{1} \cdot h}{r_{u}^{2}}+\frac{1}{2}\right) \sum_{e \in \Gamma_{h}} \frac{r_{u}^{2}}{|e|}\left\|\left[\tau_{u}^{i+1}\right]\right\|_{0, e}^{2}+K U_{3} \cdot h \frac{h^{2 \min \left(r_{u}+1, s_{u}\right)-1}}{r_{u}^{2 s_{u}}} .
$$

By choosing $h$ small enough, we obtain:

$$
\mathrm{III} \leq \sum_{e \in \Gamma_{h}} \frac{r_{u}^{2}}{|e|}\left\|\left[\tau_{u}^{i+1}\right]\right\|_{0, e}^{2}+\frac{h^{2 \min \left(r_{u}+1, s_{u}\right)-1}}{r_{u}^{2 s_{u}}} .
$$

Combining the above bounds on I, II, and III, and using lemma 2.4 we arrive at

$$
\left|T_{2}^{u}\right| \leq U U_{2}\left\|\tau_{c}^{i+1}\right\|_{0, \Omega}^{2}+U U_{3} \sum_{e \in \Gamma_{h} \cup \partial \Omega_{\mathrm{ver}}} \frac{r_{u}^{2}}{|e|}\left\|\left[\tau_{u}^{i+1}\right]\right\|_{0, e}^{2}+U U_{4}\left(\frac{h^{2 \min \left(r_{u}+1, s_{u}\right)-1}}{r_{u}^{2 s_{u}}}+\frac{h^{2 \min \left(r_{c}+1, s_{c}\right)}}{r_{c}^{2 s_{c}}}\right) .
$$

To bound the term $T_{3}^{u}$, we use the Cauchy-Schwarz inequality, Young's inequality and the inequality (2.6) which yield

$$
\left|T_{3}^{u}\right| \leq U U_{5}\left\|\tau_{c}^{i+1}\right\|_{0, \Omega}^{2}+U U_{6} \sum_{e \in \partial \Omega_{\mathrm{ver}}} \frac{r_{u}^{2}}{|e|}\left\|\left[\tau_{u}^{i+1}\right]\right\|_{0, e}^{2} .
$$

The term $T_{4}^{u}$ is bounded with the help of Cauchy-Schwarz inequality, Young's inequality, and the approximation inequality (2.2):

$$
\left|T_{4}^{u}\right| \leq \varepsilon_{2}^{u u}\left\|\tau_{u}^{i+1}\right\|_{0, \Omega}^{2}+U U_{7} \frac{h^{2 \min \left(r_{u}+1, s_{u}\right)}}{r_{u}^{2 s_{u}}}
$$

Using similar techniques as for (6.52) to obtain

$$
\left|T_{5}^{u}\right| \leq \varepsilon_{3}^{u u}\left\|\tau_{u}^{i+1}\right\|_{0, \Omega}^{2}+\widetilde{U U_{8}} \sum_{e \in \Gamma_{h} \cup \partial \Omega_{\text {ver }}} \frac{r_{u}^{2}}{|e|}\left\|\left[\tau_{u}^{i+1}\right]\right\|_{0, e}^{2}+U U_{8} \frac{h^{2 \min \left(r_{c}+1, s_{c}\right)-2}}{r_{c}^{2 s_{c}-3}} .
$$

The term $T_{6}^{u}$ is bounded using the Cauchy-Schwarz inequality, the trace inequality (2.4), and the approximation inequality (2.2):

$$
\left|T_{6}^{u}\right| \leq U U_{9} \sum_{e \in \partial \Omega_{\mathrm{ver}}} \frac{r_{u}^{2}}{|e|}\left\|\left[\tau_{u}^{i+1}\right]\right\|_{0, e}^{2}+U U_{10} \frac{h^{2 \min \left(r_{c}+1, s_{c}\right)}}{r_{c}^{2 s_{c}}} .
$$

The last term $T_{7}^{u}$ is bounded using the approximation result (2.3). Hence,

$$
\left|T_{7}^{u}\right| \leq U U_{11} \sum_{e \in \Gamma_{h} \cup \partial \Omega_{\mathrm{ver}}} \frac{r_{u}^{2}}{|e|}\left\|\left[\tau_{u}^{i+1}\right]\right\|_{0, e}^{2}+U U_{12} \frac{h^{2 \min \left(r_{u}+1, s_{u}\right)-2}}{r_{u}^{2 s_{u}-3}} .
$$

After obtaining the estimates (9.52)-(9.59), we plug them into (6.46) and use the assumption $h<1, r>1$, assumption (3.2), choose the penalty parameters large enough, choose an appropriate scaling and use the estimate (6.44) for the $\left\|\tau_{c}^{n+1}\right\|$ to obtain

$\left\|\tau_{u}^{n+1}\right\|_{0, \Omega}^{2} \leq \widetilde{C_{u}}\left(\frac{h^{2 \min \left(r_{\rho}+1, s_{\rho}\right)-4}}{r_{\rho}^{2 s_{\rho}-7}}+\frac{h^{2 \min \left(r_{c}+1, s_{c}\right)-4}}{r_{c}^{2 s_{c}-7}}+\frac{h^{2 \min \left(r_{u}+1, s_{u}\right)-4}}{r_{u}^{2 s_{u}-7}}+\frac{h^{2 \min \left(r_{v}+1, s_{v}\right)-4}}{r_{v}^{2 s_{v}-7}}\right)+\widetilde{C_{u}^{t}} \frac{r_{c}^{4}}{h^{2}} \Delta t^{2}$. 
The estimate (9.60) proves the induction hypothesis on $u_{\mathrm{DG}}$ (6.12) for $n+1$ by making the appropriate choice of $\Delta t=O\left(\frac{h^{2}}{r_{c}^{4}}\right)$ :

$$
\left\|\tau_{u}^{n+1}\right\|_{0, \Omega} \leq C_{u}^{\star} \frac{h}{r_{\min }^{2}}
$$

\subsection{Proof of Lemma 7.1}

Firstly, the proof of the statement (7.52) of Lemma 7.1 is the direct consequence of the induction hypothesis $S R$, and is the same as for the Lemma 6.1.

Secondly, to prove the next statement (7.53) of Lemma 7.1, let us first consider (7.30), and set $w^{\rho}=\tau_{z_{\rho}}^{i}$, to obtain:

$$
\left\|\tau_{z_{\rho}}^{i}\right\|_{0, \Omega}^{2}=\int_{\Omega} \tau_{\rho}^{i} \tau_{z_{\rho}}^{i}+M_{\rho}^{i}\left(\tau_{z_{\rho}}^{i}\right)
$$

where

$$
\begin{aligned}
M_{\rho}^{i}\left(w^{\rho}\right)=\int_{\Omega}\left(\xi_{z_{\rho}}^{i}-\xi_{\rho}^{i}\right) w^{\rho}-\Delta t\left(\sum_{E \in \mathcal{E}_{h}} \int_{E} \nabla \tau_{\rho}^{i} \nabla w^{\rho}-\sum_{e \in \Gamma_{h}} \int_{e}\left\{\nabla \tau_{\rho}^{i} \cdot \mathbf{n}_{e}\right\}\left[w^{\rho}\right]\right. \\
+\varepsilon \sum_{e \in \Gamma_{h}} \int_{e}\left\{\nabla w^{\rho} \cdot \mathbf{n}_{e}\right\}\left[\tau_{\rho}^{i}\right]+\sigma_{\rho} \sum_{e \in \Gamma_{h}} \frac{r_{\rho}^{2}}{|e|} \int_{e}\left[\tau_{\rho}^{i}\right]\left[w^{\rho}\right] \\
-\sum_{E \in \mathcal{E}_{h}} \int_{E} \chi \tau_{\rho}^{i} u_{\mathrm{DG}}^{i}\left(w_{\rho}^{i}\right)_{x}-\sum_{E \in \mathcal{E}_{h}} \int_{E} \chi \widetilde{\rho}^{i} \tau_{u}^{i}\left(w_{\rho}^{i}\right)_{x}+\sum_{E \in \mathcal{E}_{h}} \int_{E} \chi \widetilde{\rho}^{i} \xi_{u}^{i}\left(w_{\rho}^{i}\right)_{x} \\
+\sum_{e \in \Gamma_{h}^{\mathrm{ver}}} \int_{e}\left(\left(\chi \rho_{\mathrm{DG}}^{i} u_{\mathrm{DG}}^{i}\right)^{*}-\left(\chi \rho^{i} u^{i}\right)^{* *}\right) n_{x}\left[w_{\rho}^{i}\right]-\sum_{E \in \mathcal{E}_{h}} \int_{E} \chi \tau_{\rho}^{i} v_{\mathrm{DG}}^{i}\left(w_{\rho}^{i}\right)_{y}-\sum_{E \in \mathcal{E}_{h}} \int_{E} \chi \widetilde{\rho}^{i} \tau_{v}^{i}\left(w_{\rho}^{i}\right)_{y} \\
-\sum_{E \in \mathcal{E}_{h}} \int_{E} \chi \widetilde{\rho}^{i} \xi_{v}^{i}\left(w_{\rho}^{i}\right)_{y}+\sum_{e \in \Gamma_{h}^{\mathrm{hor}}} \int_{e}\left(\left(\chi \rho_{\mathrm{DG}}^{i} v_{\mathrm{DG}}^{i}\right)^{*}-\left(\chi \rho^{i} v^{i}\right)^{* *}\right) n_{y}\left[w_{\rho}^{i}\right] \\
-\sum_{E \in \mathcal{E}_{h}} \int_{E} \nabla \xi_{\rho}^{i} \nabla w^{\rho}+\sum_{e \in \Gamma_{h}} \int_{e}\left\{\nabla \xi_{\rho}^{i} \cdot \mathbf{n}_{e}\right\}\left[w^{\rho}\right]-\varepsilon \sum_{e \in \Gamma_{h}} \int_{e}\left\{\nabla w^{\rho} \cdot \mathbf{n}_{e}\right\}\left[\xi_{\rho}^{i}\right] \\
\left.-\sigma_{\rho} \sum_{e \in \Gamma_{h}} \frac{r_{\rho}^{2}}{|e|} \int_{e}\left[\xi_{\rho}^{i}\right]\left[w^{\rho}\right]+\sum_{E \in \mathcal{E}_{h}} \int_{E} \chi \xi_{\rho}^{i} u^{i}\left(w^{\rho}\right)_{x}+\sum_{E \in \mathcal{E}_{h}} \int_{E} \chi \xi_{\rho}^{i} v^{i}\left(w^{\rho}\right)_{y}\right) \\
=T_{1}^{\rho}+T_{2}^{\rho}+\ldots+T_{19}^{\rho} .
\end{aligned}
$$

Recalling the definitions (7.28), (7.29) of $\xi_{\rho}^{i}$ and $\xi_{z_{\rho}}^{i}$, we can then easily obtain the following bound, which will be used several times throughout the error analysis:

$$
\left\|\xi_{\rho}^{i+1}-\xi_{\rho}^{i}\right\|_{0, \Omega}^{2}+\left\|\xi_{z_{\rho}}^{i}-\xi_{\rho}^{i}\right\|_{0, \Omega}^{2} \leq C_{\xi \rho} \frac{h^{2 \min \left(r_{\rho}+1, s_{\rho}\right)}}{r_{\rho}^{2 s_{\rho}}} \Delta t^{2}
$$

where positive constant $C_{\rho}$ depends on $\partial_{t} u$ and is independent of $i, h$ and $r$ (similar bound is valid for the concentration $c$ which will be used in the derivation of the error estimate for $c$ ). Using techniques similar to the estimation of the terms $T T_{1}^{\rho}-T T_{20}^{\rho},(9.2)-(9.21)$ and applying the already verified result (7.52), we obtain: 


$$
\begin{aligned}
& \left|T_{1}^{\rho}\right| \leq \varepsilon_{1}\left\|w^{\rho}\right\|_{0, \Omega}^{2}+\Delta t^{2} R_{1} \frac{h^{2 \min \left(r_{\rho}+1, s_{\rho}\right)}}{r_{\rho}^{2 s_{\rho}}} \\
& \left|T_{2}^{\rho}\right| \leq \varepsilon_{2}\left\|w^{\rho}\right\|_{0, \Omega}^{2}+R_{2} \frac{\Delta t^{2} r_{\rho}^{8}}{h^{4}}\left\|\tau_{\rho}^{i}\right\|_{0, \Omega}^{2} \\
& \left|T_{3}^{\rho}\right| \leq \varepsilon_{3}\left\|w^{\rho}\right\|_{0, \Omega}^{2}+R_{3} \frac{\Delta t^{2} r_{\rho}^{8}}{h^{4}}\left\|\tau_{\rho}^{i}\right\|_{0, \Omega}^{2} \\
& \left|T_{4}^{\rho}\right| \leq \varepsilon_{4}\left\|w^{\rho}\right\|_{0, \Omega}^{2}+R_{4} \frac{\Delta t^{2} r_{\rho}^{8}}{h^{4}}\left\|\tau_{\rho}^{i}\right\|_{0, \Omega}^{2} \\
& \left|T_{5}^{\rho}\right| \leq \varepsilon_{5}\left\|w^{\rho}\right\|_{0, \Omega}^{2}+R_{5} \frac{\Delta t^{2} r_{\rho}^{8}}{h^{4}}\left\|\tau_{\rho}^{i}\right\|_{0, \Omega}^{2} \\
& \left|T_{6}^{\rho}\right| \leq \varepsilon_{6}\left\|w^{\rho}\right\|_{0, \Omega}^{2}+R_{6} \frac{\Delta t^{2} r_{\rho}^{4}}{h^{2}}\left\|\tau_{\rho}^{i}\right\|_{0, \Omega}^{2} \\
& \left|T_{7}^{\rho}\right| \leq \varepsilon_{7}\left\|w^{\rho}\right\|_{0, \Omega}^{2}+R_{7} \frac{\Delta t^{2} r_{\rho}^{8}}{h^{4}} \frac{h^{2}}{r_{u}^{4}}\left\|\tau_{u}^{i}\right\|_{0, \Omega}^{2} \\
& \left|T_{8}^{\rho}\right| \leq \varepsilon_{8}\left\|w^{\rho}\right\|_{0, \Omega}^{2}+R_{8} \frac{\Delta t^{2} r_{\rho}^{4}}{h^{2}} \frac{h^{2 \min \left(r_{u}+1, s_{u}\right)}}{r_{u}^{2 s_{u}}} \\
& \left|T_{9}^{\rho}\right| \leq \varepsilon_{9}\left\|w^{\rho}\right\|_{0, \Omega}^{2}+R_{9} \frac{\Delta t^{2} r_{\rho}^{4}}{h^{2}}\left\|\tau_{\rho}^{i}\right\|_{0, \Omega}^{2}+R_{10} \frac{\Delta t^{2} r_{\rho}^{8}}{h^{4}} \frac{h^{2}}{r_{u}^{4}}\left\|\tau_{u}^{i}\right\|_{0, \Omega}^{2} \\
& +R_{11} \frac{\Delta t^{2} r_{\rho}^{4}}{h^{2}}\left(\frac{h^{2 \min \left(r_{\rho}+1, s_{\rho}\right)}}{r_{\rho}^{2 s_{\rho}}}+\frac{h^{2 \min \left(r_{u}+1, s_{u}\right)}}{r_{u}^{2 s_{u}}}\right) \text {. } \\
& \left|T_{10}^{\rho}\right| \leq \varepsilon_{10}\left\|w^{\rho}\right\|_{0, \Omega}^{2}+R_{12} \frac{\Delta t^{2} r_{\rho}^{4}}{h^{2}}\left\|\tau_{\rho}^{i}\right\|_{0, \Omega}^{2} \\
& \left|T_{11}^{\rho}\right| \leq \varepsilon_{11}\left\|w^{\rho}\right\|_{0, \Omega}^{2}+R_{13} \frac{\Delta t^{2} r_{\rho}^{8}}{h^{4}} \frac{h^{2}}{r_{v}^{4}}\left\|\tau_{v}^{i}\right\|_{0, \Omega}^{2} \\
& \left|T_{12}^{\rho}\right| \leq \varepsilon_{12}\left\|w^{\rho}\right\|_{0, \Omega}^{2}+R_{14} \frac{\Delta t^{2} r_{\rho}^{4}}{h^{2}} \frac{h^{2 \min \left(r_{v}+1, s_{v}\right)}}{r_{v}^{2 s_{v}}} \\
& \left|T_{13}^{\rho}\right| \leq \varepsilon_{13}\left\|w^{\rho}\right\|_{0, \Omega}^{2}+R_{15} \frac{\Delta t^{2} r_{\rho}^{4}}{h^{2}}\left\|\tau_{\rho}^{i}\right\|_{0, \Omega}^{2}+R_{16} \frac{\Delta t^{2} r_{\rho}^{8}}{h^{4}} \frac{h^{2}}{r_{v}^{4}}\left\|\tau_{v}^{i}\right\|_{0, \Omega}^{2} \\
& +R_{17} \frac{\Delta t^{2} r_{\rho}^{4}}{h^{2}}\left(\frac{h^{2 \min \left(r_{\rho}+1, s_{\rho}\right)}}{r_{\rho}^{2 s_{\rho}}}+\frac{h^{2 \min \left(r_{v}+1, s_{v}\right)}}{r_{v}^{2 s_{v}}}\right) . \\
& \left|T_{14}^{\rho}\right| \leq \varepsilon_{14}\left\|w^{\rho}\right\|_{0, \Omega}^{2}+R_{18} \frac{\Delta t^{2} r_{\rho}^{4}}{h^{2}} \frac{h^{2 \min \left(r_{\rho}+1, s_{\rho}\right)-2}}{r_{\rho}^{2 s_{\rho}-2}} \\
& \left|T_{15}^{\rho}\right| \leq \varepsilon_{15}\left\|w^{\rho}\right\|_{0, \Omega}^{2}+R_{19} \frac{\Delta t^{2} r_{\rho}^{4}}{h^{2}} \frac{h^{2 \min \left(r_{\rho}+1, s_{\rho}\right)-2}}{r_{\rho}^{2 s_{\rho}-2}}
\end{aligned}
$$




$$
\begin{aligned}
& \left|T_{16}^{\rho}\right| \leq \varepsilon_{16}\left\|w^{\rho}\right\|_{0, \Omega}^{2}+R_{19} \frac{\Delta t^{2} r_{\rho}^{5}}{h^{2}} \frac{h^{2 \min \left(r_{\rho}+1, s_{\rho}\right)-2}}{r_{\rho}^{2 s_{\rho}-2}} \\
& \left|T_{17}^{\rho}\right| \leq \varepsilon_{17}\left\|w^{\rho}\right\|_{0, \Omega}^{2}+R_{20} \frac{\Delta t^{2} r_{\rho}^{5}}{h^{2}} \frac{h^{2 \min \left(r_{\rho}+1, s_{\rho}\right)-2}}{r_{\rho}^{2 s_{\rho}-2}} \\
& \left|T_{18}^{\rho}\right| \leq \varepsilon_{18}\left\|w^{\rho}\right\|_{0, \Omega}^{2}+R_{21} \Delta t^{2} r_{\rho}^{2} \frac{h^{2 \min \left(r_{\rho}+1, s_{\rho}\right)-2}}{r_{\rho}^{2 s_{\rho}-2}} \\
& \left|T_{19}^{\rho}\right| \leq \varepsilon_{19}\left\|w^{\rho}\right\|_{0, \Omega}^{2}+R_{22} \Delta t^{2} r_{\rho}^{2} \frac{h^{2 \min \left(r_{\rho}+1, s_{\rho}\right)-2}}{r_{\rho}^{2 s_{\rho}-2}}
\end{aligned}
$$

Now combining the estimates (9.65)-(9.83), using the assumption that $h<1, r>1, \Delta t<1$, and plugging them into (9.63), we obtain the following estimate for $M_{\rho}^{i}\left(w^{\rho}\right)$ :

$$
\begin{aligned}
\left|M_{\rho}^{i}\left(w^{\rho}\right)\right| \leq \varepsilon\left\|w^{\rho}\right\|_{0, \Omega}^{2}+ & M_{1} \frac{\Delta t^{2} r_{\rho}^{8}}{h^{4}}\left\|\tau_{\rho}^{i}\right\|_{0, \Omega}^{2}+M_{2} \frac{\Delta t^{2} r_{\rho}^{8}}{h^{4}} \frac{h^{2}}{r_{u}^{4}}\left\|\tau_{u}^{i}\right\|_{0, \Omega}^{2}+M_{3} \frac{\Delta t^{2} r_{\rho}^{8}}{h^{4}} \frac{h^{2}}{r_{v}^{4}}\left\|\tau_{v}^{i}\right\|_{0, \Omega}^{2} \\
& +M_{4} \frac{\Delta t^{2} r_{\rho}^{5}}{h^{2}}\left(\frac{h^{2 \min \left(r_{\rho}+1, s_{\rho}\right)-2}}{r_{\rho}^{2 s_{\rho}-2}}+\frac{h^{2 \min \left(r_{u}+1, s_{u}\right)}}{r_{u}^{2 s_{u}}}+\frac{h^{2 \min \left(r_{v}+1, s_{v}\right)}}{r_{v}^{2 s_{v}}}\right)
\end{aligned}
$$

Next, choosing $w^{\rho}=\tau_{z_{\rho}}^{i}$ in (9.84), making $\varepsilon$ small enough, taking $\Delta t \leq C \frac{h^{2}}{r_{\rho}^{4}}$, and using the induction hypothesis $S R$ (7.50) and (7.51), we conclude that we obtain from (9.62)

$$
\left\|\tau_{z_{\rho}}^{i}\right\|_{0, \Omega}^{2} \leq \widetilde{C_{\rho}} \frac{h^{4}}{r_{\min }^{8}}
$$

As with estimate (9.85), it can be shown that

$$
\left\|\tau_{z_{c}}^{i}\right\|_{0, \Omega}^{2} \leq \widetilde{C_{c}} \frac{h^{4}}{r_{\text {min }}^{8}}
$$

Now, let us consider the equation of (7.38), it can be shown using the same techniques as in the derivation of (9.84) and (9.60):

$$
\begin{aligned}
\left|M_{u}^{i}\left(w^{u}\right)\right| \leq \varepsilon\left\|\tau_{z_{u}}^{i}\right\|_{0, \Omega}^{2}+ & M_{u}^{1} \frac{r_{c}^{4}}{h^{2}}\left\|\tau_{z_{c}}^{i}\right\|_{0, \Omega}^{2}+M_{u}^{2} \sum_{e \in \Gamma_{h} \cup \partial \Omega_{\mathrm{ver}}} \frac{r_{u}^{2}}{|e|}\left\|\left[\tau_{z_{u}}^{i}\right]\right\|_{0, e}^{2} \\
& +M_{u}^{3}\left(\frac{h^{2 \min \left(r_{c}+1, s_{c}\right)-2}}{r_{c}^{2 s_{c}-3}}+\frac{h^{2 \min \left(r_{u}+1, s_{u}\right)-2}}{r_{u}^{2 s_{u}-3}}\right)
\end{aligned}
$$

Considering $w^{u}=\tau_{z_{u}}^{i}$, making $\varepsilon$ small enough, taking penalty parameter $\sigma_{u}$ large enough, and using result (9.86), we obtain from (7.38) that:

$$
\left\|\tau_{z_{u}}^{i}\right\|_{0, \Omega}^{2} \leq \widetilde{C_{u}} \frac{h^{2}}{r_{\min }^{4}}
$$

In the same way, it can be shown that:

$$
\left\|\tau_{z_{v}}^{i}\right\|_{0, \Omega}^{2} \leq \widetilde{C_{v}} \frac{h^{2}}{r_{\min }^{4}}
$$

Applying these estimates (9.88) and (9.89), the inverse inequality, and the use of Lemma 2.1, concludes the proof of Lemma 7.1. 


\subsection{Proof of Theorem 7.2}

First let us consider, (7.46), which can be rewritten in the following way:

$$
\begin{aligned}
& \left\|\tau_{\rho}^{i+1}\right\|_{0, \Omega}^{2}-\left\|\tau_{\rho}^{i}\right\|_{0, \Omega}^{2}+\Delta t\left(\|\| \nabla \tau_{\rho}^{i}\|\|_{0, \Omega}^{2}+\sigma_{\rho} \sum_{e \in \Gamma_{h}} \frac{r_{\rho}^{2}}{|e|}\left\|\left[\tau_{\rho}^{i}\right]\right\|_{0, e}^{2}\right)+\Delta t\left(\|\| \nabla \tau_{z_{\rho}}^{i}\|\|_{0, \Omega}^{2}+\sigma_{z_{\rho}} \sum_{e \in \Gamma_{h}} \frac{r_{\rho}^{2}}{|e|} \|\left[\tau_{z_{\rho}}^{i} \|_{0, e}^{2}\right)\right. \\
& =\left\|\tau_{\rho}^{i+1}-\tau_{z_{\rho}}^{i}\right\|_{0, \Omega}^{2}+\widetilde{M}^{i}\left(\tau_{\rho}^{i}\right)+\widetilde{N}^{i}\left(\tau_{z_{\rho}}^{i}\right)
\end{aligned}
$$

where

$$
\begin{aligned}
& \widetilde{M}_{\rho}^{i}\left(\tau_{\rho}^{i}\right)=\int_{\Omega}\left(\xi_{z_{\rho}}^{i}-\xi_{\rho}^{i}\right) \tau_{\rho}^{i}+\left(A_{\rho}\left(\rho_{\mathrm{DG}}^{i}, u_{\mathrm{DG}}^{i}, v_{\mathrm{DG}}^{i}, \tau_{\rho}^{i}\right)-A_{\rho}\left(\rho^{i}, u^{i}, v^{i}, \tau_{\rho}^{i}\right)-\left(\left\|\left|\nabla \tau_{\rho}^{i}\left\|\left.\right|_{0, \Omega} ^{2}+\sigma_{\rho} \sum_{e \in \Gamma_{h}} \frac{r_{\rho}^{2}}{|e|}\right\|\left[\tau_{\rho}^{i}\right] \|_{0, e}^{2}\right.\right.\right.\right. \\
& \widetilde{N}_{\rho}^{i}\left(\tau_{z_{\rho}}^{i}\right)=\int_{\Omega}\left(2 \xi_{\rho}^{i+1}-\xi_{\rho}^{i}-\xi_{z_{\rho}}^{i}-2 E(x, y, i)\right) \tau_{z_{\rho}}^{i}+\left(A_{\rho}\left(z_{\mathrm{DG}, \rho}^{i}, z_{\mathrm{DG}, \mathrm{u}}^{i}, z_{\mathrm{DG}, \mathrm{v}}^{i}, \tau_{z_{\rho}}^{i}\right)-A_{\rho}\left(z_{\rho}^{i}, z_{u}^{i}, z_{v}^{i}, \tau_{z_{\rho}}^{i}\right)\right. \\
& \left.-\left(\left\|\left|\nabla \tau_{z_{\rho}}^{i}\right|\right\|_{0, \Omega}^{2}+\sigma_{z_{\rho}} \sum_{e \in \Gamma_{h}} \frac{r_{\rho}^{2}}{|e|}\left\|\left[\tau_{z_{\rho}}^{i}\right]\right\|_{0, e}^{2}\right)\right) \Delta t
\end{aligned}
$$

Let us estimate, the terms on the RHS of (9.90), starting with terms $\widetilde{M}$ and $\widetilde{N}$. To estimate these terms, instead of techniques used to obtain estimates in (9.84), we will use techniques similar to the ones used in estimating the terms $T_{2}^{\rho}-T_{10}^{\rho}$ and $T_{13}^{\rho}-T_{18}^{\rho}$ in (6.17) (including Lemma 6.1). Thus, we will obtain the following bounds for the $\widetilde{M}_{\rho}^{i}\left(\tau_{\rho}^{i}\right)$ :

$$
\begin{aligned}
& \left|\widetilde{M}_{\rho}^{i}\left(\tau_{\rho}^{i}\right)\right| \leq \widetilde{M}_{1} \Delta t\left\|\tau_{\rho}^{i}\right\|_{0, \Omega}^{2}+\widetilde{\varepsilon}_{M} \Delta t\left(\|\| \nabla \tau_{\rho}^{i} \mid\left\|_{0, \Omega}^{2}+\widetilde{C}_{M} \sum_{e \in \Gamma_{h}} \frac{r_{\rho}^{2}}{|e|}\right\|\left[\tau_{\rho}^{i}\right] \|_{0, e}^{2}\right)+\widetilde{M}_{2} \Delta t\left\|\tau_{u}^{i}\right\|_{0, \Omega}^{2}+\widetilde{M}_{3} \Delta t\left\|\tau_{v}^{i}\right\|_{0, \Omega}^{2} \\
& +\widetilde{M}_{4} \Delta t\left(\frac{h^{2 \min \left(r_{\rho}+1, s_{\rho}\right)-2}}{r_{\rho}^{2 s_{\rho}-3}}+\frac{h^{2 \min \left(r_{u}+1, s_{u}\right)}}{r_{u}^{2 s_{u}}}+\frac{h^{2 \min \left(r_{v}+1, s_{v}\right)}}{r_{v}^{2 s_{v}}}\right)
\end{aligned}
$$

Similarly, we can derive the following estimate $\widetilde{N}_{\rho}^{i}\left(\tau_{z_{\rho}}^{i}\right)$ (applying now Lemma 7.1):

$$
\begin{aligned}
& \left|\widetilde{N}_{\rho}^{i}\left(\tau_{z_{\rho}}^{i}\right)\right| \leq \widetilde{N}_{1} \Delta t\left\|\tau_{z_{\rho}}^{i}\right\|_{0, \Omega}^{2}+\widetilde{\varepsilon}_{N} \Delta t\left(\left|\left\|\nabla \tau_{z_{\rho}}^{i} \mid\right\|_{0, \Omega}^{2}+\widetilde{C}_{N} \sum_{e \in \Gamma_{h}} \frac{r_{\rho}^{2}}{|e|}\left\|\left[\tau_{z_{\rho}}^{i}\right]\right\|_{0, e}^{2}\right)+\widetilde{N}_{3} \Delta t\left\|\tau_{z_{u}}^{i}\right\|_{0, \Omega}^{2}+\widetilde{N}_{4} \Delta t\left\|\tau_{z_{v}}^{i}\right\|_{0}^{2}\right. \\
& +\widetilde{N}_{5} \Delta t\left(\frac{h^{2 \min \left(r_{\rho}+1, s_{\rho}\right)-2}}{r_{\rho}^{2 s_{\rho}-3}}+\frac{h^{2 \min \left(r_{u}+1, s_{u}\right)}}{r_{u}^{2 s_{u}}}+\frac{h^{2 \min \left(r_{v}+1, s_{v}\right)}}{r_{v}^{2 s_{v}}}\right)+O\left(\Delta t^{5}\right)
\end{aligned}
$$

Next, we need to bound the first term of the RHS (9.90). First, let us notice that by subtracting the equation (7.30) from the equation (7.31), and setting $w^{\rho}=\tau_{\rho}^{i+1}-\tau_{z_{\rho}}^{i}$, we obtain that the first term on the RHS of (9.84) which can be expressed as :

$$
\left\|\tau_{\rho}^{i+1}-\tau_{z_{\rho}}^{i}\right\|_{0, \Omega}^{2}=\frac{1}{2}\left(N_{\rho}^{i}\left(\tau_{\rho}^{i+1}-\tau_{z_{\rho}}^{i}\right)-M_{\rho}^{i}\left(\tau_{\rho}^{i+1}-\tau_{z_{\rho}}^{i}\right)\right)
$$

Hence, in order to estimate $\left\|\tau_{\rho}^{i+1}-\tau_{z_{\rho}}^{i}\right\|_{0, \Omega}^{2}$ we need to estimate the RHS of (9.95). It can be shown, using ideas similar to the ideas used in the estimation of the terms $T T_{1}^{\rho}-T T_{12}^{\rho}(9.2)-(9.13)$, 
and $T T_{15}^{\rho}-T T_{20}^{\rho}$ (9.16)-(9.21), that the following estimate holds:

$$
\begin{gathered}
\left\|\tau_{\rho}^{i+1}-\tau_{z_{\rho}}^{i}\right\|_{0, \Omega}^{2} \leq C_{1}^{M N} \frac{\Delta t^{2} r_{\rho}^{4}}{h^{2}}\left(\left\|\left|\nabla \tau_{\rho}^{i}\left\|\left.\right|_{0, \Omega} ^{2}+\sum_{e \in \Gamma_{h}} \frac{r_{\rho}^{2}}{|e|}\right\|\left[\tau_{\rho}^{i}\right] \|_{0, e}^{2}\right)\right.\right. \\
+C_{2}^{M N} \frac{\Delta t^{2} r_{\rho}^{4}}{h^{2}}\left\|\tau_{\rho}^{i}\right\|_{0, \Omega}^{2}+C_{3}^{M N} \frac{\Delta t^{2} r_{\rho}^{4}}{h^{2}}\left\|\tau_{u}^{i}\right\|_{0, \Omega}^{2}+C_{4}^{M N} \frac{\Delta t^{2} r_{\rho}^{4}}{h^{2}}\left\|\tau_{v}^{i}\right\|_{0, \Omega}^{2} \\
+C_{5}^{M N} \frac{\Delta t^{2} r_{\rho}^{4}}{h^{2}}\left(\left.\left\|\nabla \tau_{z_{\rho}}^{i}\right\|\right|_{0, \Omega} ^{2}+\sum_{e \in \Gamma_{h}} \frac{r_{\rho}^{2}}{|e|}\left\|\left[\tau_{z_{\rho}}^{i}\right]\right\|_{0, e}^{2}\right) \\
+C_{6}^{M N} \frac{\Delta t^{2} r_{\rho}^{4}}{h^{2}}\left\|\tau_{z_{\rho}}^{i}\right\|_{0, \Omega}^{2}+C_{7}^{M N} \frac{\Delta t^{2} r_{\rho}^{4}}{h^{2}}\left\|\tau_{z_{u}}^{i}\right\|_{0, \Omega}^{2}+C_{8}^{M N} \frac{\Delta t^{2} r_{\rho}^{4}}{h^{2}}\left\|\tau_{z_{v}}^{i}\right\|_{0, \Omega}^{2} \\
+C_{3}^{M N} \frac{\Delta t^{2} r_{\rho}^{4}}{h^{2}}\left(\frac{h^{2 \min \left(r_{\rho}+1, s_{\rho}\right)-2}}{r_{\rho}^{2 s_{\rho}-3}}+\frac{h^{2 \min \left(r_{u}+1, s_{u}\right)}}{r_{u}^{2 s_{u}}}+\frac{h^{2 \min \left(r_{v}+1, s_{v}\right)}}{r_{v}^{2 s_{v}}}\right)+O\left(\Delta t^{5}\right)
\end{gathered}
$$

At this point, we need to get estimates for $\tau_{z_{u}}^{i}$ and $\tau_{z_{v}}^{i}$. Consider equation in (7.38). To obtain an estimate for $\tau_{z_{u}}^{i}$ in terms of $\tau_{z_{c}}^{i}$, we apply similar techniques as in (6.46) to the terms $T_{1}^{u}-T_{7}^{u}$ (6.47)-(6.55). Thus, we obtain the following estimate:

$$
\begin{gathered}
\left\|\tau_{z_{u}}^{i}\right\|_{0, \Omega}^{2}+\sum_{e \in \Gamma_{h} \cup \partial \Omega_{\mathrm{ver}}} \frac{r_{u}^{2}}{|e|}\left\|\left[\tau_{z_{u}}^{i}\right]\right\|_{0, e}^{2} \\
\leq C_{z_{u}}^{1}\left(\left\|\left|\nabla \tau_{z_{c}}^{i}\right|\right\|_{0, \Omega}^{2}+\sum_{e \in \Gamma_{h}} \frac{r_{c}^{2}}{|e|}\left\|\left[\tau_{z_{c}}^{i}\right]\right\|_{0, e}^{2}\right)+C_{z_{u}}^{2}\left(\frac{h^{2 \min \left(r_{c}+1, s_{c}\right)-2}}{r_{c}^{2 s_{c}-3}}+\frac{h^{2 \min \left(r_{u}+1, s_{u}\right)-2}}{r_{u}^{2 s_{u}-3}}\right) .
\end{gathered}
$$

Similarly, for $\tau_{z_{v}}^{i}$ we have:

$$
\begin{gathered}
\left\|\tau_{z_{v}}^{i}\right\|_{0, \Omega}^{2}+\sum_{e \in \Gamma_{h} \cup \partial \Omega_{\mathrm{hor}}} \frac{r_{v}^{2}}{|e|}\left\|\left[\tau_{z_{v}}^{i}\right]\right\|_{0, e}^{2} \\
\leq C_{z_{v}}^{1}\left(\left\|\nabla \tau_{z_{c}}^{i} \mid\right\|_{0, \Omega}^{2}+\sum_{e \in \Gamma_{h}} \frac{r_{c}^{2}}{|e|}\left\|\left[\tau_{z_{c}}^{i}\right]\right\|_{0, e}^{2}\right)+C_{z_{v}}^{2}\left(\frac{h^{2 \min \left(r_{c}+1, s_{c}\right)-2}}{r_{c}^{2 s_{c}-3}}+\frac{h^{2 \min \left(r_{v}+1, s_{v}\right)-2}}{r_{v}^{2 s_{v}-3}}\right) .
\end{gathered}
$$

Next, let us plug the estimate (9.97) and (9.98) into (9.96), and after simple modifications we obtain:

$$
\begin{gathered}
\left\|\tau_{\rho}^{i+1}-\tau_{z_{\rho}}^{i}\right\|_{0, \Omega}^{2} \leq \widetilde{C}_{1}^{M N} \frac{\Delta t^{2} r_{\rho}^{4}}{h^{2}}\left(\left\|\mid \nabla \tau_{\rho}^{i}\right\|\left\|_{0, \Omega}^{2}+\sum_{e \in \Gamma_{h}} \frac{r_{\rho}^{2}}{|e|}\right\|\left[\tau_{\rho}^{i}\right] \|_{0, e}^{2}\right) \\
+\widetilde{C}_{2}^{M N} \frac{\Delta t^{2} r_{\rho}^{4}}{h^{2}}\left\|\tau_{\rho}^{i}\right\|_{0, \Omega}^{2}+\widetilde{C}_{3}^{M N} \frac{\Delta t^{2} r_{\rho}^{4}}{h^{2}}\left\|\tau_{u}^{i}\right\|_{0, \Omega}^{2}+\widetilde{C}_{4}^{M N} \frac{\Delta t^{2} r_{\rho}^{4}}{h^{2}}\left\|\tau_{v}^{i}\right\|_{0, \Omega}^{2} \\
\widetilde{C}_{5}^{M N} \frac{\Delta t^{2} r_{\rho}^{4}}{h^{2}}\left(\|\| \nabla \tau_{z_{\rho}}^{i}\left|\left\|\left.\right|_{0, \Omega} ^{2}+\sum_{e \in \Gamma_{h}} \frac{r_{\rho}^{2}}{|e|}\right\|\left[\tau_{z_{\rho}}^{i}\right] \|_{0, e}^{2}\right)\right. \\
+\widetilde{C}_{6}^{M N} \frac{\Delta t^{2} r_{\rho}^{4}}{h^{2}}\left(\left(\left\|\nabla \tau_{z_{c}}^{i}\right\|\left\|_{0, \Omega}^{2}+\sum_{e \in \Gamma_{h}} \frac{r_{c}^{2}}{|e|}\right\|\left[\tau_{z_{c}}^{i}\right] \|_{0, e}^{2}\right)+\widetilde{C}_{7}^{M N} \frac{\Delta t^{2} r_{\rho}^{4}}{h^{2}}\left\|\tau_{z_{\rho}}^{i}\right\|_{0, \Omega}^{2}\right.
\end{gathered}
$$


$+\widetilde{C}_{3}^{M N} \frac{\Delta t^{2} r_{\rho}^{4}}{h^{2}}\left(\frac{h^{2 \min \left(r_{\rho}+1, s_{\rho}\right)-2}}{r_{\rho}^{2 s_{\rho}-3}}+\frac{h^{2 \min \left(r_{c}+1, s_{c}\right)-2}}{r_{c}^{2 s_{c}-3}}+\frac{h^{2 \min \left(r_{u}+1, s_{u}\right)-2}}{r_{u}^{2 s_{u}-3}}+\frac{h^{2 \min \left(r_{v}+1, s_{v}\right)-2}}{r_{v}^{2 s_{v}-3}}\right)+O\left(\Delta t^{5}\right)$

Next, we combine estimate (9.94), (9.93) and (9.99), plug them into (9.90) and use the assumption that $\Delta t \leq 1, h<1, r>1$ to obtain (after some simplifications) the following:

$$
\begin{aligned}
& \left\|\tau_{\rho}^{i+1}\right\|_{0, \Omega}^{2}-\left\|\tau_{\rho}^{i}\right\|_{0, \Omega}^{2}+\Delta t\left(\|\| \nabla \tau_{\rho}^{i}\|\|_{0, \Omega}^{2}+\sigma_{\rho} \sum_{e \in \Gamma_{h}} \frac{r_{\rho}^{2}}{|e|}\left\|\left[\tau_{\rho}^{i}\right]\right\|_{0, e}^{2}\right)+\Delta t\left(\|\| \nabla \tau_{z_{\rho}}^{i}\|\|_{0, \Omega}^{2}+\sigma_{z_{\rho}} \sum_{e \in \Gamma_{h}} \frac{r_{\rho}^{2}}{|e|}\left\|\left[\tau_{z_{\rho}}^{i}\right]\right\|_{0, e}^{2}\right) \\
& \leq C_{1}^{r} \frac{\Delta t^{2} r_{\rho}^{4}}{h^{2}}\left(\left\|\mid \nabla \tau_{\rho}^{i}\right\|\left\|_{0, \Omega}^{2}+\sum_{e \in \Gamma_{h}} \frac{r_{\rho}^{2}}{|e|}\right\|\left[\tau_{\rho}^{i}\right] \|_{0, e}^{2}\right)+C_{2}^{r} \frac{\Delta t^{2} r_{\rho}^{4}}{h^{2}}\left\|\tau_{\rho}^{i}\right\|_{0, \Omega}^{2}+C_{3}^{r} \frac{\Delta t^{2} r_{\rho}^{4}}{h^{2}}\left\|\tau_{u}^{i}\right\|_{0, \Omega}^{2} \\
& +C_{4}^{r} \frac{\Delta t^{2} r_{\rho}^{4}}{h^{2}}\left\|\tau_{v}^{i}\right\|_{0, \Omega}^{2}+C_{5}^{r} \frac{\Delta t^{2} r_{\rho}^{4}}{h^{2}}\left(\left\|\mid \nabla \tau_{z_{\rho}}^{i}\right\|\left\|_{0, \Omega}^{2}+\sum_{e \in \Gamma_{h}} \frac{r_{\rho}^{2}}{|e|}\right\|\left[\tau_{z_{\rho}}^{i}\right] \|_{0, e}^{2}\right) \\
& +C_{6}^{r} \frac{\Delta t^{2} r_{\rho}^{4}}{h^{2}}\left(\left.\left\|\nabla \tau_{z_{c}}^{i}\right\|\right|_{0, \Omega} ^{2}+\sum_{e \in \Gamma_{h}} \frac{r_{c}^{2}}{|e|}\left\|\left[\tau_{z_{c}}^{i}\right]\right\|_{0, e}^{2}\right)+C_{7}^{r} \frac{\Delta t^{2} r_{\rho}^{4}}{h^{2}}\left\|\tau_{z_{\rho}}^{i}\right\|_{0, \Omega}^{2} \\
& +C_{8}^{r} \frac{\Delta t^{2} r_{\rho}^{4}}{h^{2}}\left(\frac{h^{2 \min \left(r_{\rho}+1, s_{\rho}\right)-2}}{r_{\rho}^{2 s_{\rho}-3}}+\frac{h^{2 \min \left(r_{c}+1, s_{c}\right)-2}}{r_{c}^{2 s_{c}-3}}+\frac{h^{2 \min \left(r_{u}+1, s_{u}\right)-2}}{r_{u}^{2 s_{u}-3}}+\frac{h^{2 \min \left(r_{v}+1, s_{v}\right)-2}}{r_{v}^{2 s_{v}-3}}\right) \\
& +O\left(\Delta t^{5}\right)
\end{aligned}
$$

Next, consider the error equations (7.47). Using similar techniques as for the estimates in the equation (7.46), we obtain the following estimate for $\tau^{c}$ :

$$
\begin{aligned}
& \left\|\tau_{c}^{i+1}\right\|_{0, \Omega}^{2}-\left\|\tau_{c}^{i}\right\|_{0, \Omega}^{2}+\Delta t\left(\left.\left\|\left|\nabla \tau_{c}^{i}\right|\right\|\right|_{0, \Omega} ^{2}+\sigma_{c} \sum_{e \in \Gamma_{h}} \frac{r_{c}^{2}}{|e|}\left\|\left[\tau_{c}^{i}\right]\right\|_{0, e}^{2}\right)+\Delta t\left(\left\|\left|\nabla \tau_{z_{c}}^{i}\left\|\left.\right|_{0, \Omega} ^{2}+\sigma_{z_{c}} \sum_{e \in \Gamma_{h}} \frac{r_{c}^{2}}{|e|}\right\|\left[\tau_{z_{c}}^{i}\right] \|_{0, e}^{2}\right)\right.\right. \\
& \leq C_{1}^{c} \frac{\Delta t^{2} r_{c}^{4}}{h^{2}}\left(\left\|\nabla \tau_{c}^{i}\right\|\left\|_{0, \Omega}^{2}+\sum_{e \in \Gamma_{h}} \frac{r_{c}^{2}}{|e|}\right\|\left[\tau_{c}^{i}\right] \|_{0, e}^{2}\right)+C_{2}^{c} \Delta t^{2}\left\|\tau_{c}^{i}\right\|_{0, \Omega}^{2} \\
& +C_{3}^{c} \Delta t^{2}\left\|\tau_{\rho}^{i}\right\|_{0, \Omega}^{2}+C_{4}^{c} \frac{\Delta t^{2} r_{\rho}^{4}}{h^{2}}\left(\left.\left\|\nabla \tau_{z_{c}}^{i}\right\|\right|_{0, \Omega} ^{2}+\sum_{e \in \Gamma_{h}} \frac{r_{c}^{2}}{|e|}\left\|\left[\tau_{z_{c}}^{i}\right]\right\|_{0, e}^{2}\right)+C_{5}^{c} \Delta t^{2}\left\|\tau_{z_{\rho}}^{i}\right\|_{0, \Omega}^{2} \\
& +C_{6}^{c} \Delta t^{2}\left\|\tau_{z_{c}}^{i}\right\|_{0, \Omega}^{2}+C_{7}^{c} \frac{\Delta t^{2} r_{\rho}^{4}}{h^{2}}\left(\frac{h^{2 \min \left(r_{\rho}+1, s_{\rho}\right)}}{r_{\rho}^{2 s_{\rho}}}+\frac{h^{2 \min \left(r_{c}+1, s_{c}\right)-2}}{r_{c}^{2 s_{c}-3}}\right)+O\left(\Delta t^{5}\right)
\end{aligned}
$$

Now, choose $\Delta t \leq C \frac{h^{2}}{r_{c}^{4}}$, apply lemma 2.4 to estimate $\left\|\tau_{z_{\rho}}^{i}\right\|_{0, \Omega}^{2}$, sum for $i=0, \ldots, n$, and apply discrete Gronwall's lemma to (9.101) to obtain:

$$
\begin{aligned}
& \left\|\tau_{c}^{n+1}\right\|_{0, \Omega}^{2}+\sum_{i=0}^{n} \Delta t\left(\left\|\left|\nabla \tau_{c}^{i}\right|\right\|_{0, \Omega}^{2}+\sum_{e \in \Gamma_{h}} \frac{r_{c}^{2}}{|e|}\left\|\left[\tau_{c}^{i}\right]\right\|_{0, e}^{2}\right)+\sum_{i=0}^{n} \Delta t\left(\left.\left\|\nabla \tau_{z_{c}}^{i}\right\|\right|_{0, \Omega} ^{2}+\sum_{e \in \Gamma_{h}} \frac{r_{c}^{2}}{|e|}\left\|\left[\tau_{z_{c}}^{i}\right]\right\|_{0, e}^{2}\right) \\
& \leq K_{1}^{c} \sum_{i=0}^{n} \Delta t\left\|\tau_{\rho}^{i}\right\|_{0, \Omega}^{2}+K_{2}^{c} \sum_{i=0}^{n} \Delta t\left(\left\||| \nabla \tau_{z_{\rho}}^{i}\right\|\left\|_{0, \Omega}^{2}+\sum_{e \in \Gamma_{h}} \frac{r_{\rho}^{2}}{|e|}\right\|\left[\tau_{z_{\rho}}^{i}\right] \|_{0, e}^{2}\right) \\
& +K_{3}^{c} \sum_{i=0}^{n} \Delta t\left(\frac{h^{2 \min \left(r_{\rho}+1, s_{\rho}\right)}}{r_{\rho}^{2 s_{\rho}}}+\frac{h^{2 \min \left(r_{c}+1, s_{c}\right)-2}}{r_{c}^{2 s_{c}-3}}\right)
\end{aligned}
$$




$$
+O\left(\Delta t^{4}\right)
$$

Next, summing the equation (9.100) for $i=0, \ldots n$, using the above estimate (9.102), considering $\Delta t \leq C \frac{h^{2}}{r_{\rho}^{4}}$, choosing the penalty parameters large enough, and using the assumption $\Delta t<1, h<1, r>1$, we obtain:

$$
\begin{aligned}
& \left\|\tau_{\rho}^{n+1}\right\|_{0, \Omega}^{2}+\sum_{i=0}^{n} \Delta t\left(\|\| \nabla \tau_{\rho}^{i}\left\|\left.\right|_{0, \Omega} ^{2}+\sum_{e \in \Gamma_{h}} \frac{r_{\rho}^{2}}{|e|}\right\|\left[\tau_{\rho}^{i}\right] \|_{0, e}^{2}\right)+\sum_{i=0}^{n} \Delta t\left(\left\||| \nabla \tau_{z_{\rho}}^{i} \mid\right\|_{0, \Omega}^{2}+\sum_{e \in \Gamma_{h}} \frac{r_{\rho}^{2}}{|e|}\left\|\left[\tau_{z_{\rho}}^{i}\right]\right\|_{0, e}^{2}\right) \\
& \leq R_{1} \sum_{i=0}^{n} \Delta t\left\|\tau_{\rho}^{i}\right\|_{0, \Omega}^{2}+R_{2} \sum_{i=0}^{n} \Delta t\left\|\tau_{u}^{i}\right\|_{0, \Omega}^{2}+R_{3} \sum_{i=0}^{n} \Delta t\left\|\tau_{v}^{i}\right\|_{0, \Omega}^{2} \\
& +R_{4} \sum_{i=0}^{n} \Delta t\left(\frac{h^{2 \min \left(r_{\rho}+1, s_{\rho}\right)-2}}{r_{\rho}^{2 s_{\rho}-3}}+\frac{h^{2 \min \left(r_{c}+1, s_{c}\right)-2}}{r_{c}^{2 s_{c}-3}}+\frac{h^{2 \min \left(r_{u}+1, s_{u}\right)-2}}{r_{u}^{2 s_{u}-3}}+\frac{h^{2 \min \left(r_{v}+1, s_{v}\right)-2}}{r_{v}^{2 s_{v}-3}}\right) \\
& +O\left(\Delta t^{4}\right)
\end{aligned}
$$

Now, applying induction hypothesis $S R(7.48)$, (7.49) and using discrete Gronwall's lemma we obtain:

$$
\begin{aligned}
& \left\|\tau_{\rho}^{n+1}\right\|_{0, \Omega}^{2}+\sum_{i=0}^{n} \Delta t\left(\left\||| \nabla \tau_{\rho}^{i}\left|\left\|\left.\right|_{0, \Omega} ^{2}+\sum_{e \in \Gamma_{h}} \frac{r_{\rho}^{2}}{|e|}\right\|\left[\tau_{\rho}^{i}\right] \|_{0, e}^{2}\right)+\sum_{i=0}^{n} \Delta t\left(\left\|\left|\nabla \tau_{z_{\rho}}^{i}\right|\right\|_{0, \Omega}^{2}+\sum_{e \in \Gamma_{h}} \frac{r_{\rho}^{2}}{|e|}\left\|\left[\tau_{z_{\rho}}^{i}\right]\right\|_{0, e}^{2}\right)\right.\right. \\
& \leq C\left(\frac{h^{2 \min \left(r_{\rho}+1, s_{\rho}\right)-2}}{r_{\rho}^{2 s_{\rho}-3}}+\frac{h^{2 \min \left(r_{c}+1, s_{c}\right)-2}}{r_{c}^{2 s_{c}-3}}+\frac{h^{2 \min \left(r_{u}+1, s_{u}\right)-2}}{r_{u}^{2 s_{u}-3}}+\frac{h^{2 \min \left(r_{v}+1, s_{v}\right)-2}}{r_{v}^{2 s_{v}-3}}\right) \\
& +O\left(\Delta t^{4}\right)
\end{aligned}
$$

The estimate (9.104) also confirms the induction hypothesis (7.51) for $\rho$ for $i+1=n+1$. Using the final estimate for $\tau_{\rho}^{n+1}(9.104)$ we derive the following bound for $\tau_{c}^{n+1}$ :

$$
\begin{aligned}
& \left\|\tau_{c}^{n+1}\right\|_{0, \Omega}^{2}+\sum_{i=0}^{n} \Delta t\left(\left|\left\|\nabla \tau_{c}^{i} \mid\right\|_{0, \Omega}^{2}+\sum_{e \in \Gamma_{h}} \frac{r_{c}^{2}}{|e|}\left\|\left[\tau_{c}^{i}\right]\right\|_{0, e}^{2}\right)+\sum_{i=0}^{n} \Delta t\left(\left\|\left|\nabla \tau_{z_{c}}^{i}\left\|\left.\right|_{0, \Omega} ^{2}+\sum_{e \in \Gamma_{h}} \frac{r_{c}^{2}}{|e|}\right\|\left[\tau_{z_{c}}^{i}\right] \|_{0, e}^{2}\right)\right.\right.\right. \\
& \leq C\left(\frac{h^{2 \min \left(r_{\rho}+1, s_{\rho}\right)-2}}{r_{\rho}^{2 s_{\rho}-3}}+\frac{h^{2 \min \left(r_{c}+1, s_{c}\right)-2}}{r_{c}^{2 s_{c}-3}}+\frac{h^{2 \min \left(r_{u}+1, s_{u}\right)-2}}{r_{u}^{2 s_{u}-3}}+\frac{h^{2 \min \left(r_{v}+1, s_{v}\right)-2}}{r_{v}^{2 s_{v}-3}}\right) \\
& +O\left(\Delta t^{4}\right)
\end{aligned}
$$

Again, the above estimate (9.105) confirms the induction hypothesis (7.51) for $c$, for $i+1=n$. Next, to obtain the estimate for $\tau_{u}^{n+1}$ we consider second error equation in (7.39). Employing similar techniques as in the case of the error equation (6.46) and multiplying by $\Delta t$, it can be shown that:

$$
\begin{gathered}
\sum_{i=-1}^{n} \Delta t\left\|\tau_{u}^{i+1}\right\|_{0, \Omega}^{2}+C_{1}^{u} \sum_{i=-1}^{n} \Delta t \sum_{e \in \Gamma_{h} \cup \partial \Omega_{\mathrm{ver}}} \frac{r_{u}^{2}}{|e|}\left\|\left[\tau_{u}^{i+1}\right]\right\|_{0, e}^{2} \\
\leq C_{2}^{u}\left(\frac{\Delta t r_{c}^{4}}{h^{2}}\left\|\tau_{c}^{n+1}\right\|_{0, \Omega}^{2}+\frac{\Delta t r_{c}^{4}}{h^{2}}\left\|\tau_{c}^{n+1}\right\|_{0, \Omega}^{2}\right) \\
+C_{3}^{u} \sum_{i=0}^{n} \Delta t\left(\left\|\left|\nabla \tau_{c}^{i}\right|\right\|_{0, \Omega}^{2}+\sum_{e \in \Gamma_{h}} \frac{r_{c}^{2}}{|e|}\left\|\left[\tau_{c}^{i}\right]\right\|_{0, e}^{2}\right)+C_{4}^{u} \sum_{i=0}^{n} \Delta t\left(\frac{h^{2 \min \left(r_{c}+1, s_{c}\right)-2}}{r_{c}^{2 s_{c}-3}}+\frac{h^{2 \min \left(r_{u}+1, s_{u}\right)-2}}{r_{u}^{2 s_{u}-3}}\right) .
\end{gathered}
$$


Final estimate is obtained by choosing $\Delta t \leq C \frac{h^{2}}{r_{c}^{4}}$ and by using the estimate for $\tau_{c}^{n+1}(9.105)$ :

$$
\begin{gathered}
\sum_{i=-1}^{n} \Delta t\left\|\tau_{u}^{i+1}\right\|_{0, \Omega}^{2}+\sum_{i=-1}^{n} \Delta t \sum_{e \in \Gamma_{h} \cup \partial \Omega_{\mathrm{ver}}} \frac{r_{u}^{2}}{|e|}\left\|\left[\tau_{u}^{i+1}\right]\right\|_{0, e}^{2} \\
\leq U\left(\frac{h^{2 \min \left(r_{\rho}+1, s_{\rho}\right)-2}}{r_{\rho}^{2 s_{\rho}-3}}+\frac{h^{2 \min \left(r_{c}+1, s_{c}\right)-2}}{r_{c}^{2 s_{c}-3}}+\frac{h^{2 \min \left(r_{u}+1, s_{u}\right)-2}}{r_{u}^{2 s_{u}-3}}+\frac{h^{2 \min \left(r_{v}+1, s_{v}\right)-2}}{r_{v}^{2 s_{v}-3}}\right)+U_{t} \Delta t^{4} .
\end{gathered}
$$

The estimate (9.107) proves the induction hypothesis (7.48) for $i+1=n+1$ :

$$
\begin{gathered}
\sum_{i=0}^{n+1} \Delta t\left\|\tau_{u}^{i}\right\|_{0, \Omega}^{2} \leq U\left(\frac{h^{2 \min \left(r_{\rho}+1, s_{\rho}\right)-2}}{r_{\rho}^{2 s_{\rho}-3}}+\frac{h^{2 \min \left(r_{c}+1, s_{c}\right)-2}}{r_{c}^{2 s_{c}-3}}\right. \\
\left.+\frac{h^{2 \min \left(r_{u}+1, s_{u}\right)-2}}{r_{u}^{2 s_{u}-3}}+\frac{h^{2 \min \left(r_{v}+1, s_{v}\right)-2}}{r_{v}^{2 s_{v}-3}}\right)+U_{t} \Delta t^{4}
\end{gathered}
$$

Induction hypothesis $S R$ (7.50) can be shown using similar techniques as in the proof of the induction hypothesis $S$ (6.12). Similarly, we prove the induction hypothesis $S R$ for $\tau_{v}$ and show that:

$$
\begin{gathered}
\sum_{i=0}^{n} \Delta t\left\|\tau_{v}^{i+1}\right\|_{0, \Omega}^{2}+\sum_{i=0}^{n} \Delta t \sum_{e \in \Gamma_{h} \cup \partial \Omega_{\mathrm{hor}}} \frac{r_{v}^{2}}{|e|}\left\|\left[\tau_{v}^{i+1}\right]\right\|_{0, e}^{2} \\
\leq V\left(\frac{h^{2 \min \left(r_{\rho}+1, s_{\rho}\right)-2}}{r_{\rho}^{2 s_{\rho}-3}}+\frac{h^{2 \min \left(r_{c}+1, s_{c}\right)-2}}{r_{c}^{2 s_{c}-3}}+\frac{h^{2 \min \left(r_{u}+1, s_{u}\right)-2}}{r_{u}^{2 s_{u}-3}}+\frac{h^{2 \min \left(r_{v}+1, s_{v}\right)-2}}{r_{v}^{2 s_{v}-3}}\right)+V_{t} \Delta t^{4} .
\end{gathered}
$$

Acknowledgment: The research of Y.Epshteyn is based upon work supported by the Center for Nonlinear Analysis (CNA) under the National Science Foundation Grant \# DMS-0635983.

\section{References}

[1] J. Adlen, Chemotaxis in bacteria, Ann. Rev. Biochem., 44 (1975), pp. 341-356.

[2] S. Agmon, Lectures on Elliptic Boundary Value Problems, Van Nostrand, Princeton, NJ, 1965.

[3] D.N. Arnold, An interior penalty finite element method with discontinuous elements, SIAM J. Numer. Anal., 19 (1982), pp. 742-760.

[4] I. BABUŠKA AND M. SuRI, The h-p version of the finite element method with quasiuniform meshes, RAIRO Modél. Math. Anal. Numér., 21 (1987), pp. 199-238.

[5] I. BABUŠKA AND M. SURI, The optimal convergence rates of the p-version of the finite element method, SIAM J. Numer. Anal., 24 (1987), pp. 750-776.

[6] J.T. Bonner, The cellular slime molds, Princeton University Press, Princeton, New Jersey, 2nd ed., 1967. 
[7] S. Brenner, Poincaré-Friedrichs inequalities for piecewise $H^{1}$ functions, SIAM J. Numer. Anal., 41 (2003), pp. 306-324.

[8] E.O. Budrene And H.C. Berg, Complex patterns formed by motile cells of escherichia coli, Nature, 349 (1991), pp. 630-633.

[9] E.O. Budrene And H.C. Berg, Dynamics of formation of symmetrical patterns by chemotactic bacteria, Nature, 376 (1995), pp. 49-53.

[10] A. Chertock And A. Kurganov, A positivity preserving central-upwind scheme for chemotaxis and haptotaxis models, Numer. Math. submitted.

[11] S. Childress And J.K. Percus, Nonlinear aspects of chemotaxis, Math. Biosc., 56 (1981), pp. 217-237.

[12] B. Cockburn, G. Kanschat, and D. Schotzau, A note on discontinuous Galerkin divergence-free solutions of the Navier-Stokes equations, Journal of Scientific Computing, 31 (2007), pp. 61-73.

[13] B. Cockburn, G.E. Karniadakis, and C.-W. Shu, eds., First International Symposium on Discontinuous Galerkin Methods, vol. 11 of Lecture Notes in Computational Science and Engineering, Springer-Verlag, 2000.

[14] B. Cockburn And C.-W. Shu., The local discontinuous Galerkin method for convectiondiffusion systems, SIAM J. Numer. Anal., 35 (1998), pp. 2440-2463.

[15] M.H. Cohen And A. Robertson, Wave propagation in the early stages of aggregation of cellular slime molds, J. Theor. Biol., 31 (1971), pp. 101-118.

[16] J. Douglas And T. Dupont, Lecture Notes in Physics, vol. 58, Springer-Verlag, 1976, ch. Interior penalty procedures for elliptic and parabolic Galerkin methods.

[17] Y. Epshteyn, Discontinuous Galerkin methods for the chemotaxis and haptotaxis models, Journal of Computational and Applied Mathematics, 224 (2008), pp. 168-181.

[18] Y. Epshteyn and A. Kurganov, New Interior Penalty Discontinuous Galerkin Methods for the Keller-Segel Chemotaxis Model, Siam J. Numer. Anal., 47 (2008), pp. 386-408. also CNA report, http://www.math.cmu.edu/cna/pub2007.html.

[19] Y. Epshteyn AND B. Rivière, On the solution of incompressible two-phase flow by a $p$ version discontinuous Galerkin method, Comm. Numer. Methods Engrg., 22 (2006), pp. 741751.

[20] Y. Epshteyn And B. Rivière, Convergence of High Order Methods for Miscible Displacement, International Journal of Numerical Analysis and Modeling, 5, Supp (2008), pp. 47-63.

[21] F. Filbet, A finite volume scheme for the Patlak-Keller-Segel chemotaxis model, Numer. Math., 104 (2006), pp. 457-488. 
[22] V. Girault, B. Rivière, And M.F. Wheeler, A discontinuous Galerkin method with non-overlapping domain decomposition for the Stokes and Navier-Stokes problems, Math. Comp., 74 (2005), pp. 53-84.

[23] M.A. Herrero And J.J.L. VelázQuez, A blow-up mechanism for a chemotaxis model, Ann. Scuola Normale Superiore, 24 (1997), pp. 633-683.

[24] D. Horstmann, From 1970 until now: The Keller-Segel model in chemotaxis and its consequences I, Jahresber. DMV, 105 (2003), pp. 103-165.

[25] D. Horstmann, From 1970 until now: The Keller-Segel model in chemotaxis and its consequences II, Jahresber. DMV, 106 (2004), pp. 51-69.

[26] E.F. Keller And L.A. Segel, Initiation of slime mold aggregation viewed as an instability, J. Theor. Biol., 26 (1970), pp. 399-415.

[27] E.F. Keller And L.A. Segel, Model for chemotaxis, J. Theor. Biol., 30 (1971), pp. 225234.

[28] E.F. Keller And L.A. Segel, Traveling bands of chemotactic bacteria: A theoretical analysis, J. Theor. Biol., 30 (1971), pp. 235-248.

[29] A. Kurganov And C.-T. Lin, On the reduction of numerical dissipation in central-upwind schemes, Commun. Comput. Phys., 2 (2007), pp. 141-163.

[30] A. Kurganov, S. Noelle, And G. Petrova, Semi-discrete central-upwind schemes for hyperbolic conservation laws and Hamilton-Jacobi equations, SIAM J. Sci. Comput., 23 (2001), pp. 707-740.

[31] A. Kurganov And G. Petrova, Central-upwind schemes on triangular grids for hyperbolic systems of conservation laws, Numer. Methods Partial Differential Equations, 21 (2005), pp. 536-552.

[32] A. MARrocco, 2D simulation of chemotaxis bacteria aggregation, M2AN Math. Model. Numer. Anal., 37 (2003), pp. 617-630.

[33] V. Nanjundiah, Chemotaxis, signal relaying and aggregation morphology, J. Theor. Biol., 42 (1973), pp. 63-105.

[34] C.S. Patlak, Random walk with persistence and external bias, Bull. Math: Biophys., 15 (1953), pp. 311-338.

[35] L.M. Prescott, J.P. Harley, And D.A. Klein, Microbiology, Wm. C. Brown Publishers, Chicago, London, 3rd ed., 1996.

[36] B. Rivière, M.F. Wheeler, And V. Girault., A priori error estimates for finite element methods based on discontinuous approximation spaces for elliptic problems, SIAM J. Numer. Anal., 39 (2001), pp. 902-931.

[37] C. Schwab, $p$ - and hp-finite element methods, Numerical Mathematics and Scientific Computation, (1998). The Clarendon Press, Oxford University Press, New York. 
[38] S. Sun AND M.F. WheELER, Symmetric and nonsymmetric discontinuous Galerkin methods for reactive transport in porous media, SIAM J. Numer. Anal., 43 (2005), pp. 195-219.

[39] R. Tyson, S.R. Lubkin, and J.D. Murray, A minimal mechanism for bacterial pattern formation, Proc. Roy. Soc. Lond. B, 266 (1999), pp. 299-304.

[40] R. Tyson, L.G. Stern, And R.J. LeVeque, Fractional step methods applied to a chemotaxis model, J. Math. Biol., 41 (2000), pp. 455-475.

[41] Q. Zhang And C-W. Shu, Error Estimates to Smooth Solutions of Runge-Kutta Discontinuous Galerkin Methods for Scalar Conservation Laws, SIAM J. Numer. Anal., 42 (2004), pp. 641-666. 

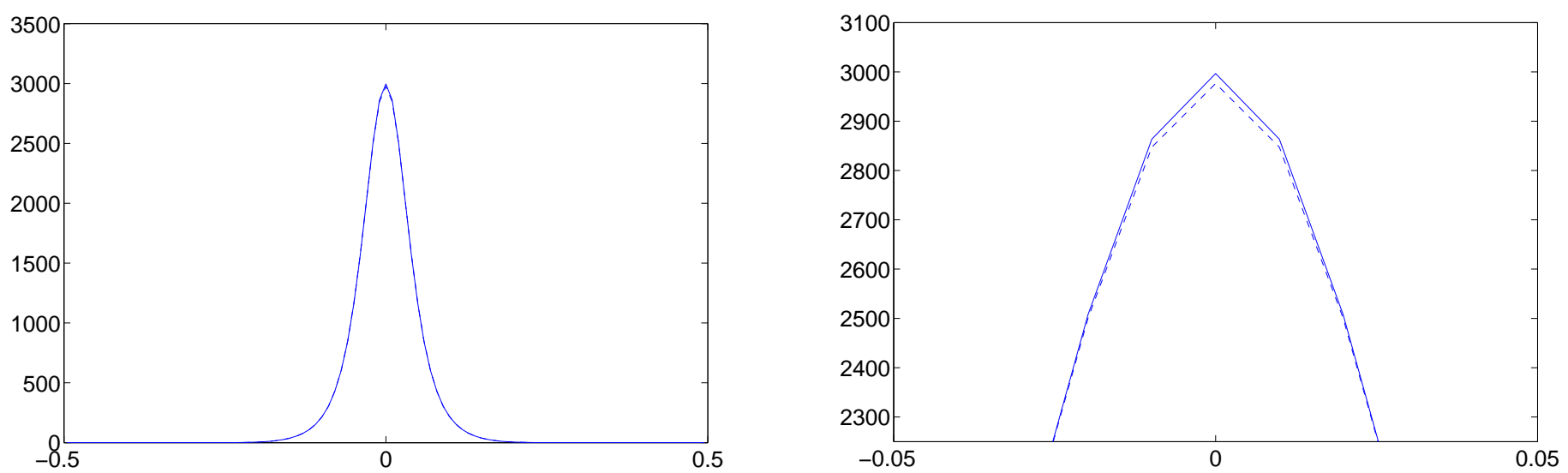

Figure 8.1: contours along the line $\mathcal{L}$ of the density $\rho$ on $101 \times 101$ grid using quadratic polynomial approximation $p=2$ at time $t=5 \times 10^{-6}$ (left column) and zoom view of the same contours(right column). Forward Euler - dashed line, Runge - Kutta - solid line.
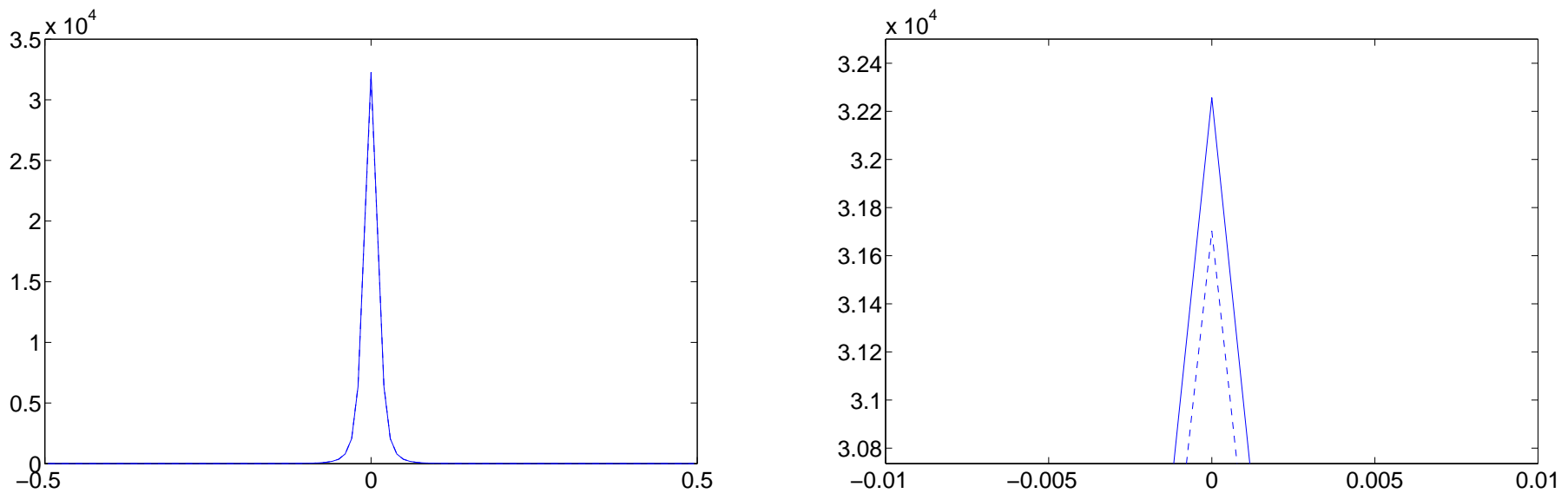

Figure 8.2: contours along the line $\mathcal{L}$ of the density $\rho$ on $101 \times 101$ grid using quadratic polynomial approximation $p=2$ at later time $t=2 \times 10^{-5}$ (left column) and zoom view of the same contours(right column). Forward Euler - dashed line, Runge - Kutta - solid line. 

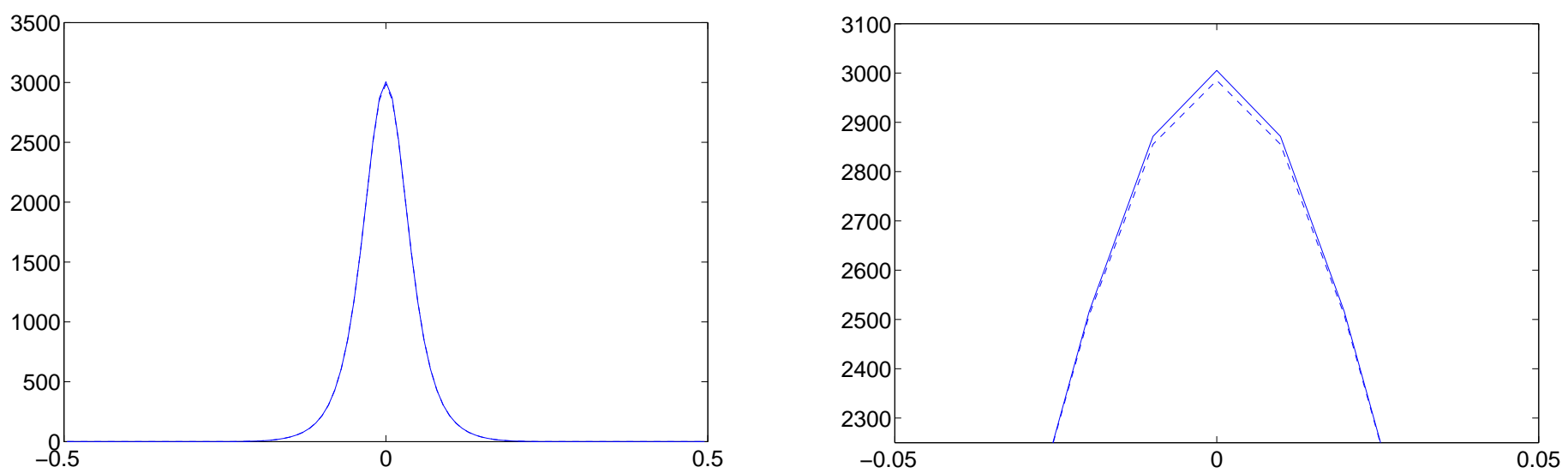

Figure 8.3: contours along the line $\mathcal{L}$ of the density $\rho$ on $101 \times 101$ grid using cubic polynomial approximation $p=3$ at time $t=5 \times 10^{-6}$ (left column) and zoom view of the same contours(right column). Forward Euler - dashed line, Runge - Kutta - solid line.
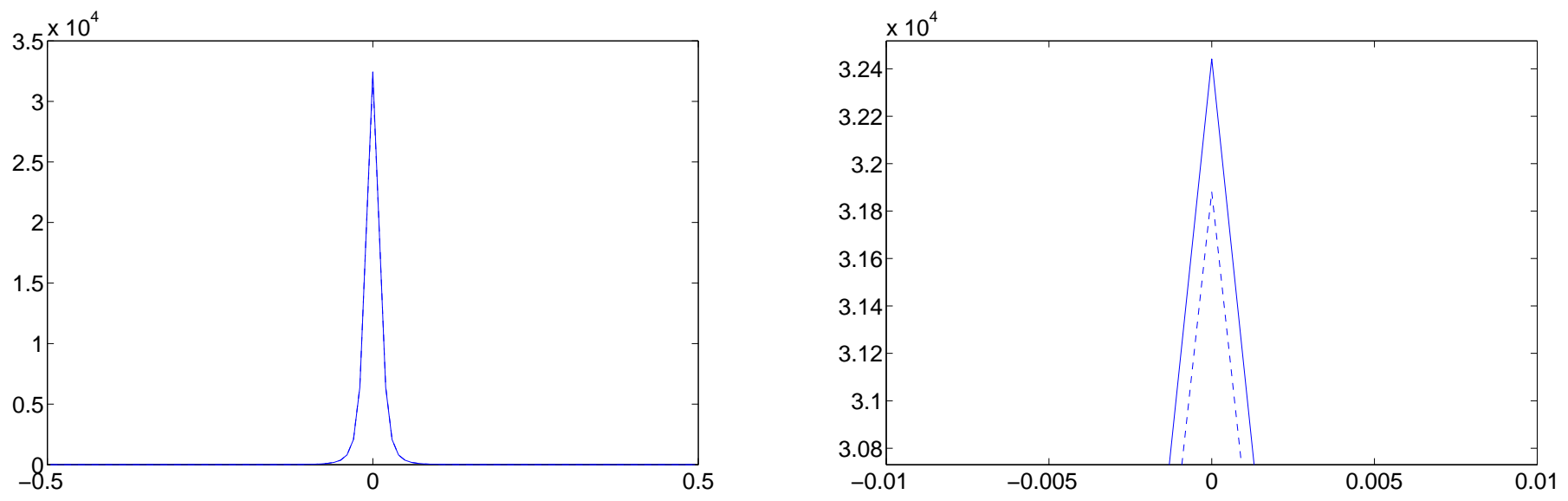

Figure 8.4: contours along the line $\mathcal{L}$ of the density $\rho$ on $101 \times 101$ grid using cubic polynomial approximation $p=3$ at later time $t=2 \times 10^{-5}$ (left column) and zoom view of the same contours(right column). Forward Euler - dashed line, Runge - Kutta - solid line. 